\title{
ASYMPTOTICS FOR THE MULTIPLE POLE SOLUTIONS OF THE NONLINEAR SCHRÖDINGER EQUATION
}

\author{
Cornelia Schiebold \\ Department of Natural Sciences, Engineering, and Mathematics \\ Mid Sweden University, S-851 70 Sundsvall, Sweden
}

\begin{abstract}
Multiple pole solutions consist of groups of weakly bound solitons. For the Nonlinear Schrödinger equation the double pole solution was constructed by Zakharov and Shabat. In the sequel particular cases have been discussed in the literature, but it has remained an open problem to understand multiple pole solutions in their full complexity.

In the present work this problem is solved, in the sense that a rigorous and complete asymptotic description of the multiple pole solutions is given. More precisely, the asymptotic paths of the solitons are determined and their position- and phase-shifts are computed explicitly. As a corollary we generalize the conservation law known for the $N$-solitons. In the special case of one wave packet, our result confirms a conjecture of Olmedilla.

Our method stems from an operator theoretic approach to integrable systems. To facilitate comparison with the literature, we also establish the link to the construction of multiple pole solutions via the Inverse Scattering Method. The work is rounded off by many examples and Mathematica plots and a detailed discussion of the transition to the next level of degeneracy.
\end{abstract}

\section{Contents}

1. Introduction 1

2. Construction of MPS's 3

\begin{tabular}{lll}
\hline 3. Main results on asymptotic behavior & 6
\end{tabular}

\begin{tabular}{llr}
\hline 4. & Geometric interpretation and examples & 9
\end{tabular}

$\begin{array}{lll}5 . & \text { Discussion of the assumptions } & 10\end{array}$

\begin{tabular}{lll}
\hline 6. & Link to ISM & 14
\end{tabular}

$\begin{array}{lll}\text { 7. Algebraic simplification of data } & 16\end{array}$

\begin{tabular}{lll}
\hline 8. Collisions of wave packets & 19
\end{tabular}

9. Collisions of the solitons within a wave packet 25

\begin{tabular}{lll}
\hline 10. & Solutions of higher degeneracy & 35
\end{tabular}

$\begin{array}{lll}\text { Appendix A. Selected computer graphics } & 41\end{array}$

\begin{tabular}{lll}
\hline Appendix B. Eigenvalue relations in the solution formula & 41
\end{tabular}

$\begin{array}{lll}\text { Appendix C. Global regularity } & 48\end{array}$

$\begin{array}{ll}\text { References } & 50\end{array}$

\section{INTRODUCTION}

One of the most distinctive features of classical integrable systems like the Korteweg-de Vries equation (KdV) or the Nonlinear Schrödinger equation (NLS) is the existence of $N$-solitons, i.e. solutions where $N$ localized waves interact in elastic 
collisions. Finding the formula for $N$-solitons $(N \geq 2)$ is nontrivial and was one of the motivations for the development of the Inverse Scattering Method (ISM), see [13, 2], 30. In the framework of ISM, $N$-solitons are obtained in the reflectionless case if the transmission coefficient has $N$ simple poles in the upper half plane. From an algebraic point of view, the next degree in complexity should correspond to multiple poles.

A priori it is not evident that this idea leads to new solutions. Actually the transmission coefficient $1 / a(k)$ of the eigenvalue equation $\left(\partial_{x}^{2}-u(x)\right) \psi=\lambda \psi\left(\lambda=k^{2}\right)$ has only simple poles on the upper imaginary axis if the potentia $u(x)$ belongs to $L^{1}(\mathbb{R})$ (see $[11$ for details). As a consequence one cannot obtain multiple pole solutions (MPS's) of the KdV by applying the ISM in the usual way for functions $u(x, t)$ which are integrable w.r.t. $x$. On the other hand, it was early observed by Zakharov and Shabat that multiple poles are possible for the NLS; in [30] they construct solutions with a wave packet of two solitons by 'coalescence' of (two simple) poles. From that time on, multiple pole solutions (MPS's) for various equations sporadically appear in the literature, see for example [12, [17, [27, [28, 29, mostly in cases of low complexity. A systematic study of the MPS's of the NLS was initiated by Olmedilla. In [15] he derives the formula for MPS's with a single wave packet consisting of $L$ solitons by solving the Gelfand-Levitan-Marchenko equation for the kernel corresponding to a single pole of order $L$ at some point $k_{0}$ in the upper half plane (see Section 6 for details). Then he proceeds to an asymptotic analysis which finally reduces to the evaluation of a determinant whose complexity increases with $L$. The evaluation is only performed for $L=2$ or 3 . For $L \leq 9$, the author refers to computer computations and conjectures a formula for $L$ arbitrary.

The main result of the present article is a complete and rigorous asymptotic analysis of the MPS's of the NLS

$$
\mathrm{i} q_{t}=q_{x x}+2 q|q|^{2} .
$$

As a first major step towards this goal, we need formulas which nicely reflect the decomposition into wave packets. Here we can build on an operator-theoretic approach which can be trace back to Marchenko [14] and was subsequently amplified in the framework of advanced Banach space geometry (44, 8], 23], 25], see [9] for an overview). There are numerous applications relying on analysis in infinite dimensional spaces [6], 7], 22, [25].

For our purposes, the main point is a solution formula depending on a matrix parameter whose Jordan form will give access to the asymptotic of the solution. The solution formula used here was first obtained in [21, see also 25]. For related work we refer to [5, [10, [18. Returning to a subtlety indicated above, we mention that formulas analogous to those used here give a natural access to the MPS's of the KdV. In contrast to the NLS case, these contain pole-shaped antisolitons [9].

The asymptotic analysis is now performed on three levels of precision: First we will show that MPS's decompose into several wave packets situated in sectors in $(x, t)$-space. Second we examine the packets closer and discover that each of them decomposes into finitely many solitons which logarithmically drift away from a common geometric center, which itself moves with constant velocity. As in the familiar case of $N$-solitons, the collision of various entities (transition from asymptotics for $t \rightarrow-\infty$ to $t \rightarrow \infty)$ is described by position- and phase-shifts, which are explicitly computed in the third step. This computation turns out to be considerably more 
involved as for other equations ([19, 20]). Fortunately we can massively rely on our earlier results published in [24]. For one single wave packet, our result confirms the conjecture of Olmedilla.

The article is organizes as follows. After constructing determinant formulas for MPS's in Section 2, we formulate our main results on their asymptotic behavior in Section 3 Section 4 explains the underlying geometry, followed by some plots indicating the subtle interaction behavior of MPS's. Section 5 addresses the assumptions in the central Theorem 3.3 . First we point out why the various technical assumptions do not restrict generality. A crucial further assumption specifies the transition to the next level of degeneracy. This happens if there are solitons or wave packets with the same velocity but corresponding to different poles. We discuss the simplest example for this phenomenon. As mentioned above, Olmedilla's point of departure is the ISM. In Section 6 we establish the link between the two approaches and show in particular that all solutions constructed in [15] are covered by Theorem 3.3. After a parameter reduction in Section 7 , the proof of Theorem 3.3 is given in Sections 8 and 9. In Section 10 we return to solutions of higher degeneracy and sketch in the first relevant case which of their properties can be captured by asymptotic analysis. The article is concluded by three appendices with complementing material. Appendix A presents some computer plots of more complicated MPS's. In Appendix B we verify an invariance property for the eigenvalues. In Appendix C we give a rigorous proof that all solutions in Theorem 2.3 (including those of higher degeneracy) are globally regular.

Acknowledgement. Section 6 owes much to enlightening discussions with Tuncay Aktosun about the ISM.

\section{Construction of MPS's}

First we explain the construction of MPS's as used in the present work. We start with an analogue of the 1-soliton for the matrix-Nonlinear Schrödinger equation (matrix-NLS)

$$
\mathrm{i} Q_{t}=Q_{x x}+2 Q \bar{Q} Q .
$$

Here the unknown $Q=Q(x, t)$ is a function with values in the $n \times n$-matrices, and $\bar{Q}$ denotes the matrix obtained from $Q$ by taking complex conjugated entries.

As a close variant, the equation 2.1 with $\bar{Q}$ replaced by $Q^{*}$, the adjoint of $Q$, is often considered in the literature (see [1], 5], [10] and references therein). For our needs the two approaches are essentially equivalent since we mainly use them to derive handy solution formulas.

Proposition 2.1. Let $A, C \in \mathcal{M}_{n, n}(\mathbb{C})$, and define $L(x, t)=\exp \left(A x-\mathrm{i} A^{2} t\right) C=$ $e^{A x-\mathrm{i} A^{2} t} C$. Then

$$
Q=(I+L \bar{L})^{-1}(A L+L \bar{A}),
$$

where $I$ denotes the identity matrix, is a solution of the matrix-NLS 2.1) on $\left\{(x, t) \in \mathbb{R}^{2} \mid I+L \bar{L}\right.$ invertible $\}$.

Proposition 2.1 is a special case of more general solution formulas for the operatorNLS [22], see also 23] for the operator-AKNS system. For the reader's convenience, we give a direct proof. 
Proof. Using $L_{t}=-\mathrm{i} A^{2} L$ (and thus $\bar{L}_{t}=\mathrm{i} \bar{A}^{2} \bar{L}$ ), we calculate

$$
\begin{aligned}
Q_{t} & =-(I+L \bar{L})^{-1}(L \bar{L})_{t}(I+L \bar{L})^{-1}(A L+L \bar{A})+(I+L \bar{L})^{-1}(A L+L \bar{A})_{t} \\
& =\mathrm{i}(I+L \bar{L})^{-1}\left(A^{2} L-L \bar{A}^{2}\right) \bar{L}(I+L \bar{L})^{-1}(A L+L \bar{A}) \\
& -\mathrm{i}(I+L \bar{L})^{-1} A^{2}(A L+L \bar{A}) \\
& =\mathrm{i}(I+L \bar{L})^{-1}\left(\left(A^{2} L-L \bar{A}^{2}\right) \bar{L}-A^{2}(I+L \bar{L})\right)(I+L \bar{L})^{-1}(A L+L \bar{A}) \\
& =-\mathrm{i}(I+L \bar{L})^{-1}\left(A^{2}+L \bar{A}^{2} \bar{L}\right) Q .
\end{aligned}
$$

Similarly, one obtains $Q_{x}=\widehat{Q} Q$.

Using $L(I+\bar{L} L)^{-1}=(I+L \bar{L})^{-1} L, L(I+\bar{L} L)^{-1} \bar{L}=I-(I+L \bar{L})^{-1},(I+\bar{L} L)^{-1}=$ $I-\bar{L}(I+L \bar{L})^{-1} L$, and $(I+\bar{L} L)^{-1} \bar{L}=\bar{L}(I+L \bar{L})^{-1}$, we get

$$
\begin{aligned}
Q \bar{Q}= & (I+L \bar{L})^{-1}\left((A L+L \bar{A})(I+\bar{L} L)^{-1}(\overline{A L}+\bar{L} A)\right) \\
= & (I+L \bar{L})^{-1}\left(A\left(L(I+\bar{L} L)^{-1}\right) \overline{A L}+A\left(L(I+\bar{L} L)^{-1} \bar{L}\right) A\right. \\
& \left.\quad+L \bar{A}\left((I+\bar{L} L)^{-1}\right) \overline{A L}+L \bar{A}\left((I+\bar{L} L)^{-1} \bar{L}\right) A\right) \\
= & (I+L \bar{L})^{-1}\left(A\left((I+L \bar{L})^{-1} L\right) \overline{A L}+A\left(I-(I+L \bar{L})^{-1}\right) A\right. \\
& \left.\quad+L \bar{A}\left(I-\bar{L}(I+L \bar{L})^{-1} L\right) \overline{A L}+L \bar{A}\left(\bar{L}(I+L \bar{L})^{-1}\right) A\right) \\
= & (I+L \bar{L})^{-1}\left(A^{2}+L \bar{A}^{2} \bar{L}\right)-(I+L \bar{L})^{-1}(A-L \overline{A L})(I+L \bar{L})^{-1}(A-L \overline{A L}) \\
= & (I+L \bar{L})^{-1}\left(A^{2}+L \bar{A}^{2} \bar{L}\right)-\widehat{Q}^{2},
\end{aligned}
$$

where $\widehat{Q}=(I+L \bar{L})^{-1}(A-L \overline{A L})$. Moreover, $\widehat{Q}_{x}=-Q \bar{Q}$. Thus we get $Q_{x x}=$ $(\widehat{Q} Q)_{x}=-Q \bar{Q} Q+\widehat{Q}^{2} Q$, and therefore

$$
Q_{x x}+2 Q \bar{Q} Q=\left(\widehat{Q}^{2}+Q \bar{Q}\right) Q \stackrel{\sqrt[2.3 p]{-}}{-}(I+L \bar{L})^{-1}\left(A^{2}+L \bar{A}^{2} \bar{L}\right) Q=\mathrm{i} Q_{t}
$$

The solution 2.2 is a matrix generalization of the well-known 1-soliton. In fact, setting $n=1$ and $A=\alpha, C=\exp (\varphi)$, we get $L(x, t)=\ell(x, t)=\exp \left(\alpha x-\mathrm{i} \alpha^{2} t+\varphi\right)$ and

$$
\begin{aligned}
q(x, t) & =2 \operatorname{Re}(\alpha) \frac{\ell(x, t)}{1+|\ell(x, t)|^{2}} \\
& =\operatorname{Re}(\alpha) e^{\mathrm{i} \operatorname{Im}(\Gamma(x, t))} \cosh ^{-1}(\operatorname{Re}(\Gamma(x, t))),
\end{aligned}
$$

where $\Gamma(x, t)=\alpha x-\mathrm{i} \alpha^{2} t+\varphi$. Note that $\operatorname{Re}(\Gamma)=\operatorname{Re}(\alpha)(x+2 \operatorname{Im}(\alpha) t)+\operatorname{Re}(\varphi)$. Thus $|\operatorname{Re}(\alpha)|$ is the height of the soliton and $v=-2 \operatorname{Im}(\alpha)$ its velocity. Since height and velocity can be specified independently, we may have superpositions of solitons with the same velocity but different heights, see also Example 5.1 and the discussion in Section 10 .

Example 2.2. Consider the 1-solitons for the parameters $\alpha_{1}=0.5-4 \mathrm{i}$ and $\alpha_{2}=1$ and $\varphi_{1}=\varphi_{2}=0$. This gives

$$
q_{1}(x, t)=\frac{1}{2} e^{-\frac{\mathrm{i}}{4}(16 x-63 t)} \cosh ^{-1}\left(\frac{x-8 t}{2}\right),
$$



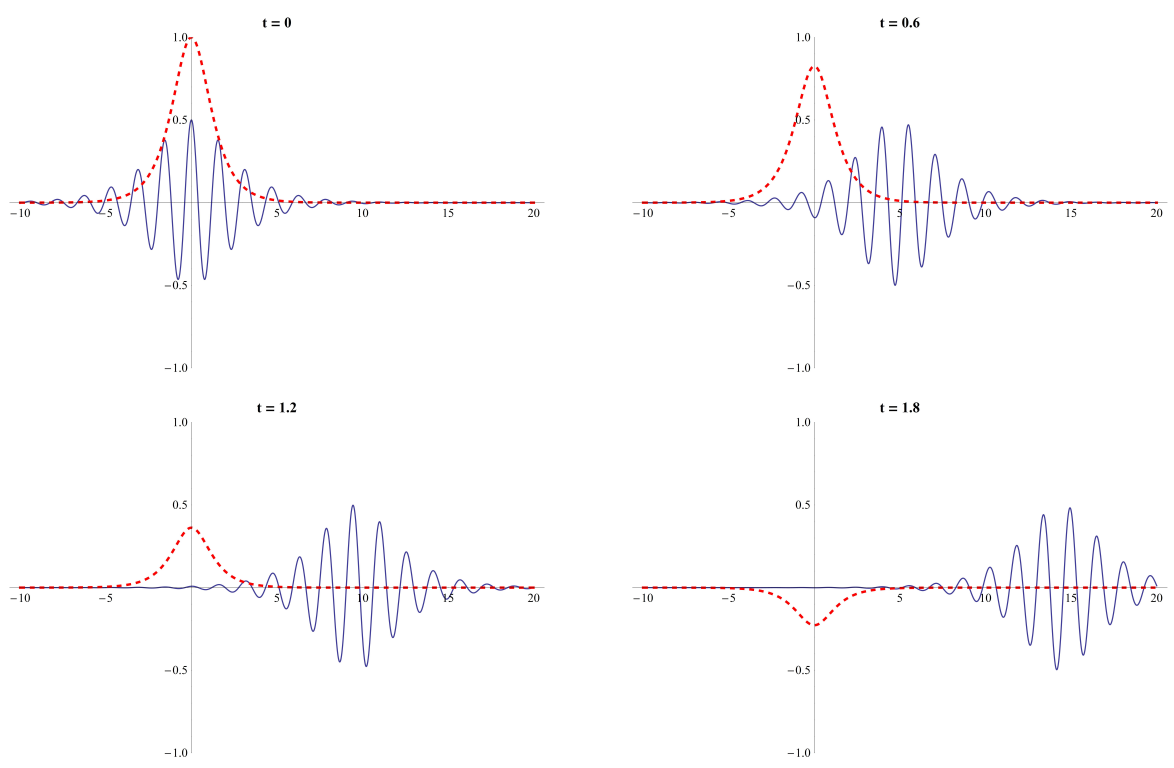

Figure 1. Snapshots of the real parts of the 1-solitons $q_{1}$ (solid) and $q_{2}$ (dotted) from Example 2.2 at the consecutive times $t=0$, $t=0.6, t=1.2$, and $t=1.8$.

$$
q_{2}(x, t)=e^{-\mathrm{i} t} \cosh ^{-1}(x) .
$$

The first soliton moves with velocity $-2 \operatorname{Im}\left(\alpha_{2}\right)=8$ and the second is stationary, see Figure 1 for an illustration.

Actually 2.2 yields much more for the scalar equation than the 1-soliton.

Theorem 2.3. Let $A \in \mathcal{M}_{n, n}(\mathbb{C})$ with $0 \notin \operatorname{spec}(A)+\operatorname{spec}(\bar{A})$, and let $a, c \in \mathbb{C}^{n}$.

a) There is a unique matrix $C \in \mathcal{M}_{n, n}(\mathbb{C})$ such that $A C+C \bar{A}=c \bar{a}^{\text {tr }}$, where $a^{\text {tr }}$ denotes the transposed of a.

b) Let $L_{0}(x, t)=\exp \left(A x-\mathrm{i} A^{2} t\right) c a^{\text {tr }}$ and $L(x, t)=\exp \left(A x-\mathrm{i} A^{2} t\right) C$ with $C$ as in a). Then the function

$$
q=1-P / p
$$

where

$$
P=\operatorname{det}\left(\begin{array}{cc}
I-L_{0} & -L \\
L & I
\end{array}\right), \quad p=\operatorname{det}\left(\begin{array}{cc}
I & -L \\
L & I
\end{array}\right),
$$

is a smooth solution of the NLS 1 (1.1) on $\mathbb{R}^{2}$.

We formulated Theorem 2.3 in the finite-dimensional setting, which is sufficient for our purposes. It should be mentioned that far reaching generalizations of Theorem 2.3 can be obtained by using the theory of determinants on quasi-Banach operator ideals, see [21] and [22, 25] for applications.

First part of the proof of Theorem 2.3. For a) it suffices to recall that the Sylvester equation $A X+X B=Y$ is uniquely solvable for every $Y$ iff $0 \notin \operatorname{spec}(A)+\operatorname{spec}(B)$ [26]. 
Here we prove b) only on the set $\Omega=\{(x, t) \mid I+L \bar{L}$ invertible $\}$ and postpone the proof that $\Omega=\mathbb{R}^{2}$ to Appendix $\mathrm{C}$. To this end, we first show that the scalar function

$$
q=a^{t r}(I+L \bar{L})^{-1} F c
$$

where $F=F(x, t)=\exp \left(A x-\mathrm{i} A^{2} t\right)$, is a solution of 1.1. Applying Proposition 2.1 , we find that

$$
Q=(I+L \bar{L})^{-1} F c \bar{a}^{t r}
$$

solves the matrix-NLS 2.1. Abbreviating $Q_{0}=(I+L \bar{L})^{-1} F$, this means that

$$
\mathrm{i} Q_{0, t} c \bar{a}^{t r}=Q_{0, x x} c \bar{a}^{t r}+2 Q_{0} c \bar{a}^{t r} \bar{Q}_{0} \bar{c} a^{t r} Q_{0} c \bar{a}^{t r} .
$$

We may assume $a \neq 0$. Choosing some vector $d$ with $\bar{a}^{t r} d=1$ and multiplying the above matrix equation with $d$ from the right, and with $a^{t r}$ from the left, we obtain

$$
\begin{aligned}
\mathrm{i} q_{t} & =\mathrm{i}\left(a^{t r} Q_{0} c\right)_{t} \\
& =\mathrm{i} a^{t r} Q_{0, t} c \\
& =a^{t r} Q_{0, x x} c+2 a^{t r} Q_{0} c \bar{a}^{t r} \bar{Q}_{0} \bar{c} a^{t r} Q_{0} c \\
& =\left(a^{t r} Q_{0} c\right)_{x x}+2\left(a^{t r} Q_{0} c\right)\left(\overline{a^{t r} Q_{0} c}\right)\left(a^{\operatorname{tr}} Q_{0} c\right) \\
& =q_{x x}+2 q|q|^{2} .
\end{aligned}
$$

Next we rewrite 2.6 in the form 2.4, 2.5). We start by observing that

$$
q=a^{t r} Q_{0} c=\operatorname{tr}\left(Q_{0} c a^{t r}\right)=1-\operatorname{det}\left(I-Q_{0} c a^{t r}\right),
$$

where we have used the fact that $\operatorname{det}(I+A)=1+\operatorname{tr}(A)$ holds for every rank-1 matrix $A$. Recall $Q_{0}=(I+L \bar{L})^{-1} F$ and $F c a^{t r}=L_{0}$. For $(x, t) \in \Omega$, this gives

$$
\begin{aligned}
q & =1-\operatorname{det}\left(I-(I+L \bar{L})^{-1} L_{0}\right) \\
& =1-\operatorname{det}\left((I+L \bar{L})^{-1}\left(I+L \bar{L}-L_{0}\right)\right) \\
& =1-\frac{\operatorname{det}\left(I+L \bar{L}-L_{0}\right)}{\operatorname{det}(I+L \bar{L})} .
\end{aligned}
$$

From this, the desired form of the solution is obtained by using

$$
\begin{aligned}
\operatorname{det}\left(\begin{array}{cc}
A & B_{1} \\
B_{2} & I
\end{array}\right) & =\operatorname{det}\left(\left(\begin{array}{cc}
A & B_{1} \\
B_{2} & I
\end{array}\right)\left(\begin{array}{cc}
I & 0 \\
-B_{2} & I
\end{array}\right)\right)=\operatorname{det}\left(\begin{array}{cc}
A-B_{1} B_{2} & B_{1} \\
0 & I
\end{array}\right) \\
& =\operatorname{det}\left(A-B_{1} B_{2}\right),
\end{aligned}
$$

which holds for every square matrix $A$.

\section{MAin RESUlts ON ASYMPtotic BeHAVIOR}

Now we turn to the asymptotics of the solutions provided by Theorem 2.3 .

We say that two functions $f=f(x, t), g=g(x, t)$ have the same asymptotic behavior for $t \rightarrow \infty(t \rightarrow-\infty)$ if for every $\epsilon>0$ there is a $t_{\epsilon}$ such that $\mid f(x, t)-$ $g(x, t) \mid<\epsilon$ for $t>t_{\epsilon}\left(t<t_{\epsilon}\right)$ and $x \in \mathbb{R}$. In this case we also write $f(x, t) \approx$ $g(x, t)$ for $t \approx \infty(t \approx-\infty)$.

We will formulate further assumptions, one essential for an asymptotic examination, the others with the mere purpose to simplify notation. Let us start with two assumptions of the second type. 
Assumption 3.1. The matrix $A \in \mathcal{M}_{n, n}(\mathbb{C})$ is in Jordan form with $N$ Jordan blocks $A_{j}$ of size $n_{j} \times n_{j}$ corresponding to the eigenvalues $\alpha_{j}$, i.e.,

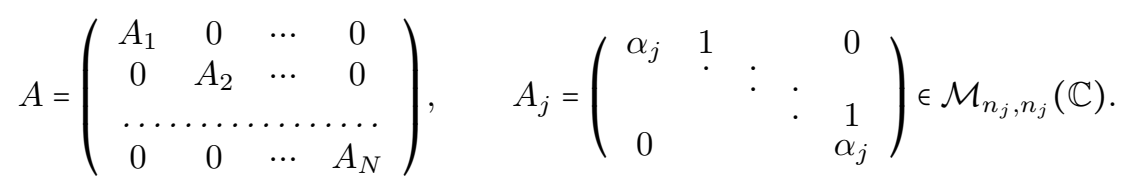

It is useful to adapt our notation to the given Jordan structure of $A$ : For a vector $c \in \mathbb{C}^{n}$, we write

$$
c=\left(c_{j}\right)_{j=1}^{N} \quad \text { with } \quad c_{j}=\left(c_{j}^{(\mu)}\right)_{\mu=1}^{n_{j}} \in \mathbb{C}^{n_{j}},
$$

and, analogously, for a matrix $T \in \mathcal{M}_{n, n}(\mathbb{C})$,

$$
T=\left(T_{i j}\right)_{i j=1}^{N} \quad \text { with } \quad T_{i j}=\left(t_{i j}^{(\mu \nu)}\right)_{\substack{\mu=1, \ldots, n_{i} \\ \nu=1, \ldots, n_{j}}} \in \mathcal{M}_{n_{i}, n_{j}}(\mathbb{C}) .
$$

Assumption 3.2. The vectors $a, c \in \mathbb{C}^{n}$ satisfy $a_{j}^{(1)} c_{j}^{\left(n_{j}\right)} \neq 0$.

In Section 5 it is shown that Assumptions 3.1 and 3.2 can be made without loss of generality. Now we are in position to state our main result.

Theorem 3.3. Let Assumptions 3.1 and 3.2 be met and suppose that

a) the $\operatorname{Re}\left(\alpha_{j}\right)$ are positive,

b) the $\operatorname{Im}\left(\alpha_{j}\right)$ are pairwise different.

To these data we associate the (soliton-like) functions

$$
q_{j j^{\prime}}^{ \pm}(x, t)=\operatorname{sgn}(t)^{n_{j}-1} \operatorname{Re}\left(\alpha_{j}\right) e^{\mathrm{i} \operatorname{Im}\left(\Gamma_{j j^{\prime}}^{ \pm}(x, t)\right)} \cosh ^{-1}\left(\operatorname{Re}\left(\Gamma_{j j^{\prime}}^{ \pm}(x, t)\right)\right)
$$

for $j^{\prime}=0, \ldots, n_{j}-1$ with

$$
\Gamma_{j j^{\prime}}^{ \pm}(x, t)=\alpha_{j} x-\mathrm{i} \alpha_{j}^{2} t \mp J^{\prime} \log |t|+\varphi_{j}+\varphi_{j}^{ \pm}+\varphi_{j j^{\prime}}^{ \pm},
$$

where we have set $J^{\prime}=-\left(n_{j}-1\right)+2 j^{\prime}$. The quantities $\varphi_{j}, \varphi_{j}^{ \pm}$are (up to integer multiples of $2 \pi \mathrm{i}$ ) defined by

$$
\begin{aligned}
& \exp \left(\varphi_{j}\right)=\mathrm{i} a_{j}^{(1)} c_{j}^{\left(n_{j}\right)} /\left(2 \mathrm{i} \operatorname{Re}\left(\alpha_{j}\right)\right)^{n_{j}}, \\
& \exp \left(\varphi_{j}^{ \pm}\right)=\prod_{k \in \Lambda_{j}^{ \pm}}\left[\frac{\alpha_{j}-\alpha_{k}}{\alpha_{j}+\bar{\alpha}_{k}}\right]^{2 n_{k}}
\end{aligned}
$$

with the index sets $\Lambda_{j}^{ \pm}=\left\{k \mid \operatorname{Im}\left(\alpha_{j}\right) \lessgtr \operatorname{Im}\left(\alpha_{k}\right)\right\}$, and the quantities $\varphi_{j j^{\prime}}^{ \pm}$are defined by

$$
\varphi_{j j^{\prime}}^{ \pm}= \pm \log \left(\left(2 \operatorname{Re}\left(\alpha_{j}\right)\right)^{-2 J^{\prime}} \frac{j^{\prime} !}{\left(j^{\prime}-J^{\prime}\right) !}\right) .
$$

Then the asymptotic behavior of the solution $q(x, t)$ associated to the data $A, a, c$ by Theorem 2.3 is

$$
q(x, t) \approx \sum_{j=1}^{N} \sum_{j^{\prime}=0}^{n_{j}-1} q_{j j^{\prime}}^{ \pm}(x, t) \quad \text { for } t \approx \pm \infty
$$


The above theorem says that the solitons $q_{j j^{\prime}}^{ \pm}(x, t)$ travel asymptotically along the logarithmic curves $\left\{(x, t) \in \mathbb{R}^{2} \mid \operatorname{Re}\left(\Gamma_{j j^{\prime}}^{ \pm}(x, t)\right)=0\right\}$, i.e. the velocity of $q_{j j^{\prime}}^{ \pm}(x, t)$ is $v_{j}=-2 \operatorname{Im}\left(\alpha_{j}\right)$, up to a logarithmic deviation. Hence, for $j^{\prime}=0, \ldots, n_{j}-1$ the $q_{j j^{\prime}}^{ \pm}(x, t)$ can be viewed as a weakly bound group or wave packet. Their oscillation is encoded by $\operatorname{Im}\left(\Gamma_{j j^{\prime}}^{ \pm}(x, t)\right)$.

The initial shift $\varphi_{j}$ determines position and phase of the geometric center of the $j$ th wave packet at a given time. Note that it is a parameter of the solution. The quantities $\varphi_{j}^{ \pm}$encode position- and phase-shifts in the asymptotic form due to collisions of wave packets with different velocities. Here the index sets $\Lambda_{j}^{+}\left(\operatorname{resp} . \Lambda_{j}^{-}\right)$ stand for those wave packets which move slower (resp. faster) than the $j$ th wave packet. The $\varphi_{j j^{\prime}}^{ \pm}$encode the positioning of the solitons within the $j$ th wave packet. Observe that the wave corresponding to $j^{\prime}=0$ moves the furthest to the left for $t \approx-\infty$ and the furthest to the right for $t \approx \infty$. This suggests that the solitons flip side during collision, a phenomenon which becomes more transparent for MPS's of the KdV. Here a packet with two waves comprises a soliton and an antisoliton (with a polar singularity) and their changing of side is manifest 9.

A detailed discussion of the assumptions is postponed to Section 5 . Here we just observe that the main purpose of a) is to simplify formulas. Actually $\operatorname{Re}\left(\alpha_{j}\right) \neq 0$ is enough, see Section 5. In contrast b) is essential, since it ensures that the wave packets travel with different velocities. However, in Sections 4 and 10 we will look at examples with eigenvalues $\alpha_{1} \neq \alpha_{2}$ satisfying $\operatorname{Im}\left(\alpha_{1}\right)=\operatorname{Im}\left(\alpha_{2}\right)$. Our observations suggest a study of such solutions in coarser terms.

For several reasons it is not straightforward to verify that our formulas include those of [15, 30. The details are provided in Remark 9.8 .

By a direct calculation, we deduce the following conservation law from 3.2 .

Corollary 3.4. The sum of all position-shifts vanishes:

$$
\sum_{j=1}^{N} n_{j}\left(\operatorname{Re}\left(\varphi_{j}^{+}\right)-\operatorname{Re}\left(\varphi_{j}^{-}\right)\right)=0 .
$$

Proof. Without loss of generality, $\operatorname{Im}\left(\alpha_{1}\right)<\ldots<\operatorname{Im}\left(\alpha_{N}\right)$. Then $\Lambda_{j}^{-}=\{1, \ldots, j-1\}$, $\Lambda_{j}^{+}=\{j+1, \ldots, N\}$, and

$$
\begin{aligned}
\varphi:=\sum_{j=1}^{N} n_{j}\left(\varphi_{j}^{+}-\varphi_{j}^{-}\right) & =2 \sum_{j=1}^{N} \sum_{k=j+1}^{N} n_{j} n_{k} \log A_{j k}-2 \sum_{j=1}^{N} \sum_{k=1}^{j-1} n_{j} n_{k} \log A_{j k} \\
& =2 \sum_{j=1}^{N} \sum_{k=j+1}^{N} n_{j} n_{k} \log A_{j k}-2 \sum_{k=1}^{N} \sum_{j=k+1}^{N} n_{j} n_{k} \log A_{j k} \\
& =2 \sum_{j=1}^{N} \sum_{k=j+1}^{N} n_{j} n_{k} \log \frac{A_{j k}}{A_{k j}}
\end{aligned}
$$

for $A_{j k}=\left(\frac{\alpha_{j}-\alpha_{k}}{\alpha_{j}+\bar{\alpha}_{k}}\right)^{2}$. Since $\frac{A_{j k}}{A_{k j}}=\left(\frac{\bar{\alpha}_{j}+\alpha_{k}}{\alpha_{j}+\bar{\alpha}_{k}}\right)^{2}$ has modulus 1, it follows $\varphi \in i \mathbb{R}$.

Remark 3.5. It is worth mentioning that the $\varphi_{j j^{\prime}}^{ \pm}$satisfy the following symmetry property which concerns the positioning of pairs of solitons which are approximately related by reflection in the geometric center of the $j$ th wave packet:

$$
\forall j^{\prime}: \quad \varphi_{j j^{\prime}}^{ \pm}=-\varphi_{j\left(n_{j}-1-j^{\prime}\right)}^{ \pm}
$$



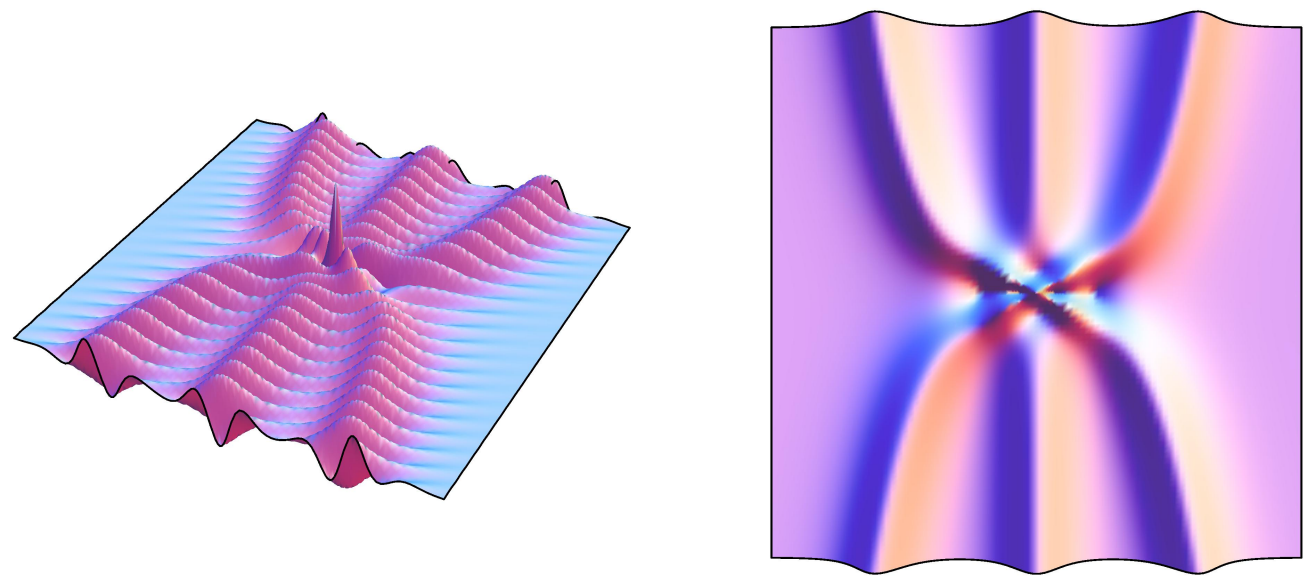

Figure 2. The wave packet from Example 4.1 consisting of three solitons. The left plot shows the real part, the right plot the modulus of the solution. Both plots use the coordinates $(x-2 t, t)$.

In particular, for $n_{j}$ odd, $\varphi_{j\left(n_{j}-1\right) / 2}^{ \pm}=0$, which means that the central soliton moves like the geometric center.

\section{Geometric interpretation and examples}

Qualitatively, Theorem 3.3 gives the following asymptotic behavior of MPS's:

Consider first a single Jordan block of size $n \times n$ with eigenvalue $\alpha$. The corresponding solution represents a wave packet of $n$ solitons whose geometric center propagates with constant velocity $v=-2 \operatorname{Im}(\alpha)$. The solitons themselves approach/drift away from the geometric center on logarithmic curves.

Hence, for $t \approx-\infty$ we can imagine each soliton to be located on one side of the center, approaching logarithmically. At some moment it changes sides, and for $t \approx \infty$ it is located on the other side of the center, drifting away logarithmically. Note that those collisions do not affect the path of the center.

Example 4.1. Figure 2 shows the solution coming from a $3 \times 3$ Jordan block with $\alpha=0.5+\mathrm{i}$. The initial shifts are encoded in $a=(1,0,0), c=(3,3,1)$. The plots show the region $-25 \leq x \leq 25,-50 \leq t \leq 50$.

The situation becomes even more transparent for MPS's of the KdV. Here in the asymptotic form regular solitons and singular antisolitons alternate, and the fact that they change side is obvious $[9]$.

In the general case of $N$ eigenvalues $\alpha_{1}, \ldots, \alpha_{N}$ with algebraic multiplicities $n_{1}, \ldots, n_{N}$, the solution is a superposition of $N$ wave packets as described above. It is remarkable that the behavior of the wave packets is a natural generalization of what is familiar for $N$-solitons: Each wave packet as a whole moves with constant velocity. Eventually, faster wave packets keep up with the slower ones and they collide. These collisions are elastic and the wave packets re-emerge unaltered suffering only position- and phase-shifts. 

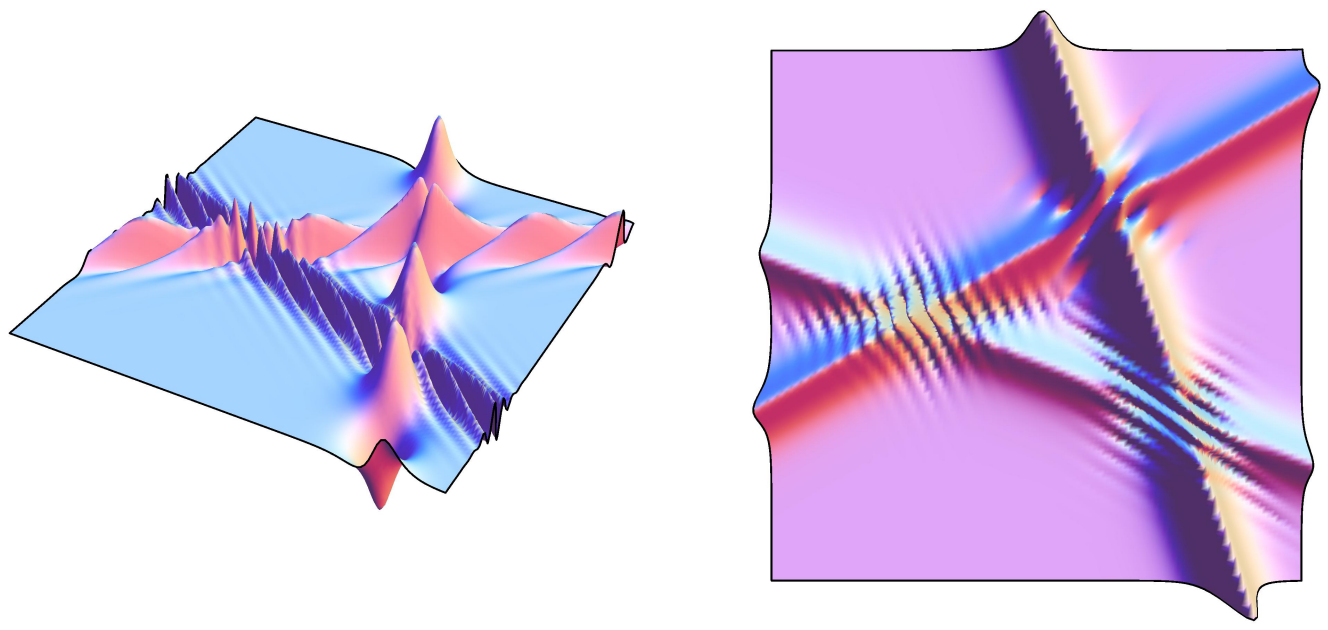

Figure 3. A 3-soliton, see Example 4.2 for details.

Example 4.2. $N$-solitons arise from $N$ simple eigenvalues. In Figure 3 we have set $N=3, \alpha_{1}=0.6+0.2 \mathrm{i}, \alpha_{2}=0.2+1.5 \mathrm{i}$, and $\alpha_{3}=0.3-\mathrm{i}$. The initial shifts are encoded in $a_{1}=a_{2}=a_{3}=1, c_{1}=1.2 e^{-2}, c_{2}=0.4 e^{10}, c_{3}=0.6$. The plots show the region $-40 \leq x \leq 30,-40 \leq t \leq 20$.

Example 4.3. Next we consider two eigenvalues $\alpha_{1}=0.4-0.5 \mathrm{i}, \alpha_{2}=0.5+0.2 \mathrm{i}$ with $n_{1}=1, n_{2}=2$. In Figure 4 the resulting solution is plotted for different choices of the parameters encoding the initial shifts (from left to right):

- $a_{1}=1, c_{1}=0.8 e^{-10}, a_{2}=(1,0), c_{2}=(2,1)$, plotted region: $-20 \leq x \leq 40$, $-40 \leq t \leq 20$

- $a_{1}=1, c_{1}=0.8, a_{2}=(1,0), c_{2}=(2,1)$, plotted region: $-30 \leq x \leq 30$, $-30 \leq t \leq 30$,

- $a_{1}=1, c_{1}=0.8 e^{10}, a_{2}=(1,0), c_{2}=(3,1)$, plotted region: $-35 \leq x \leq 25$, $-20 \leq t \leq 40$.

The diagrams (see Figures 4 and 12 14) suggest that the collision of two packets can be interpreted as the joint effect of local collisions looking like 2-solitons. Thus we obtain a physical explanation of position-shifts as sums of local shifts.

For further illustrations of MPS's we refer to Appendix A.

\section{Discussion of the Assumptions}

Now we can justify the assumptions of Theorem 3.3 .

On Assumption b) in Theorem 3.3. This assumption assures that the wave packets move with different velocities and hence can be neatly distinguished by asymptotic analysis. 

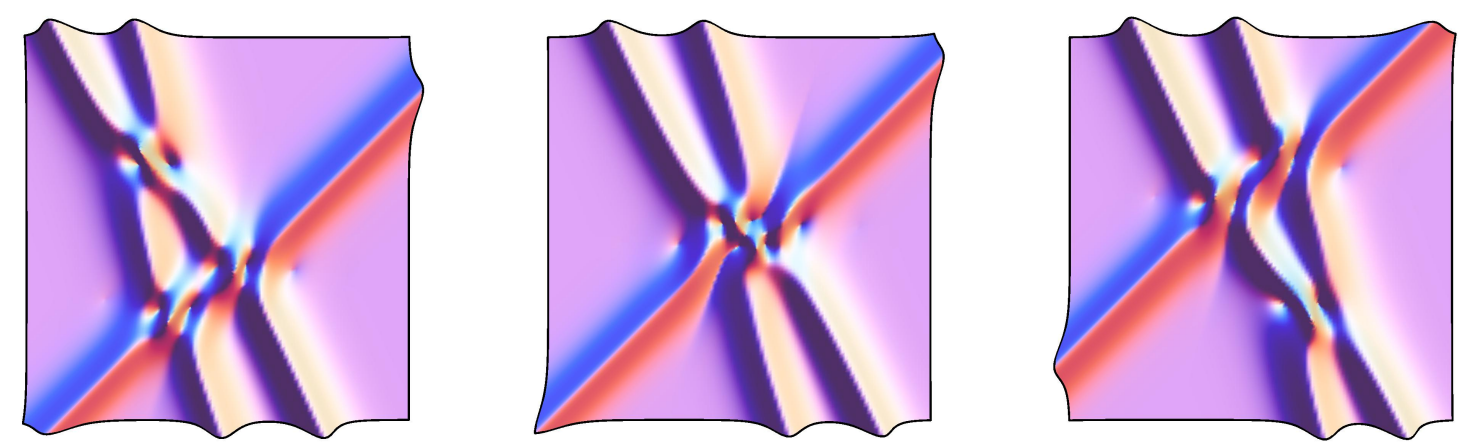

Figure 4. Collisions of a soliton with a wave packet consisting of two solitons for different choices of initial shifts, see Example 4.3 for details. In all cases the modulus of the solution is plotted.

It is instructive to look at the easiest example for which this is no longer the case, namely the superposition of two solitons with the same velocity. At first glance, one may even hesitate to consider such a solution as a 2-soliton.

Example 5.1. The data

$$
A=\left(\begin{array}{ll}
4 & 0 \\
0 & 2
\end{array}\right)
$$

yield the superposition of two stationary solitons with heights 4 and 2. Depending on the initial shifts these solutions may look quite different, see Figure 5. In the upper row the solution is displayed for $a_{1}=1, a_{2}=1, c_{1}=32, c_{2}=-18$. Note that this particular choice precisely reproduces the solution discussed in [5, Example 7.2]. In the lower row the solution is shown for $a_{1}=1, a_{2}=1, c_{1}=32 e^{2}, c_{2}=-18 e^{-1}$. Here the nature of the solution as a 2 -soliton is clearly visible. In both cases the solution is depicted for $-3 \leq x \leq 3,0 \leq t \leq 4$.

For more on degeneracy phenomena, the reader is referred to Section 10 .

On Assumption a) in Theorem 3.3. This assumption permits to realize all MPS's derived in [15] via ISM, see Section 6. Our approach allows us to formally weaken this assumption to $\operatorname{Re}\left(\alpha_{j}\right) \neq 0 \forall j$. Recall that $v_{j}=-2 \operatorname{Im}\left(\alpha_{j}\right)$. Then the only modification in Theorem 3.3 is that the index sets $\Lambda_{j}^{ \pm}$have to be replaced by

$$
\Lambda_{j}^{ \pm}=\left\{k \mid \operatorname{Re}\left(\alpha_{k}\right)>0 \text { and } v_{j} \gtrless v_{k}\right\} \cup\left\{k \mid \operatorname{Re}\left(\alpha_{k}\right)<0 \text { and } v_{j} \lessgtr v_{k}\right\} .
$$

In this context a remark concerning regularity is in order: It requires some work to prove regularity on all of $\mathbb{R}^{2}$, see Appendix $C$. Independently of that the derivation of the asymptotic behavior implicitly implies regularity for $|t|$ sufficiently large.

On Assumption 3.1. The following lemma explains why the assumption that $A$ is in Jordan form can be made without loss of generality.

Lemma 5.2. Let $A \in \mathcal{M}_{n, n}(\mathbb{C})$, and let $U$ be the matrix transforming $A$ into Jordan form $J$, i.e. $A=U^{-1} J U$. Then the solution in Theorem 2.3 is not altered if we replace simultaneously $A$ by $J$ and $a, c$ by $\left(U^{-1}\right)^{\text {tr }} a, U c$. 

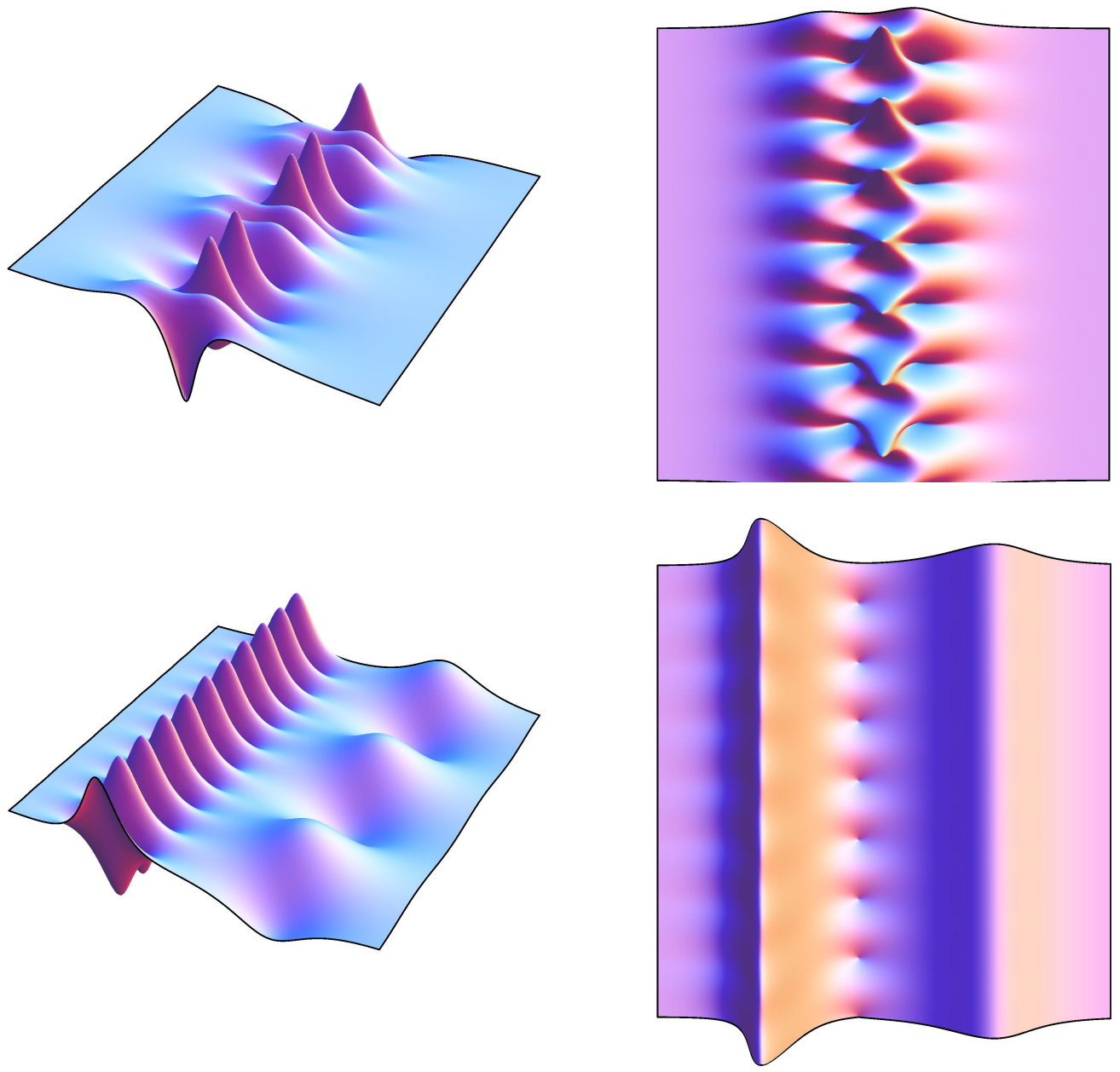

FiguRE 5. Two stationary solitons with different choices of initial position-shifts, see Example 5.1 for details. In the upper plots both solitons have approximately the same geometric center. In the lower plots this is no longer the case, and the solitons are well separated. To the left the respective real part, to the right the modulus is plotted.

Proof. Let $b=\left(U^{t r}\right)^{-1} a, d=U c$, and $D=U C \bar{U}^{-1}$. First we observe that, replacing $A$ by $J$ and $a, c$ by $b, d$ implies replacing $C$, the (unique) solution of the Sylvester equation $A X+X \bar{A}=c \bar{a}^{t r}$, by $D$. Indeed, since

$$
\begin{aligned}
d \bar{b}^{t r} & =(U c)\left(\left(\bar{U}^{-1}\right)^{t r} \bar{a}\right)^{t r}=U\left(c \bar{a}^{t r}\right) \bar{U}^{-1}=U(A C+C \bar{A}) \bar{U}^{-1} \\
& =U\left(U^{-1} J U C+C \bar{U}^{-1} \overline{J U}\right) \bar{U}^{-1}=J U C \bar{U}^{-1}+U C \bar{U}^{-1} \bar{J}=J D+D \bar{J}
\end{aligned}
$$

$D$ satisfies $J D+D \bar{J}=d \bar{b}^{t r}$. Moreover, from the very definition of the exponential function,

$$
\exp \left(A x-\mathrm{i} A^{2} t\right)=U^{-1} \exp \left(J x-\mathrm{i} J^{2} t\right) U
$$


Abbreviating $K=\exp \left(J x-\mathrm{i} J^{2} t\right) D$ and $K_{0}=\exp \left(J x-\mathrm{i} J^{2} t\right) d b^{t r}$, we thus have

$$
\begin{aligned}
L & =\exp \left(A x-\mathrm{i} A^{2} t\right) C=U^{-1} \exp \left(J x-\mathrm{i} J^{2} t\right) U\left(U^{-1} D \bar{U}\right)=U^{-1} K \bar{U}, \\
L_{0} & =\exp \left(A x-\mathrm{i} A^{2} t\right)\left(c a^{t r}\right)=U^{-1} \exp \left(J x-\mathrm{i} J^{2} t\right) U\left(\left(U^{-1} d\right)\left(U^{t r} b\right)^{t r}\right)=U^{-1} K_{0} U,
\end{aligned}
$$

yielding

$$
\begin{aligned}
P & =\operatorname{det}\left(I+\left(\begin{array}{cc}
-L_{0} & -L \\
\bar{L} & 0
\end{array}\right)\right) \\
& =\operatorname{det}\left(I+\left(\begin{array}{cc}
U^{-1} & 0 \\
0 & \bar{U}^{-1}
\end{array}\right)\left(\begin{array}{cc}
-K_{0} & -K \\
\bar{K} & 0
\end{array}\right)\left(\begin{array}{cc}
U & 0 \\
0 & \bar{U}
\end{array}\right)\right) \\
& =\operatorname{det}\left(I+\left(\begin{array}{cc}
-K_{0} & -K \\
\bar{K} & 0
\end{array}\right)\right),
\end{aligned}
$$

showing that the replacements do not change the determinant $P$. A similar, even easier calculation allows to handle $p$.

On Assumption 3.2. At first glance this assumption on the vectors $a, c$ (namely $a_{j}^{(1)} c_{j}^{\left(n_{j}\right)} \neq 0 \forall j$ ) looks artificial. However, the following lemma shows that it can indeed be made without losing anything.

Lemma 5.3. Let Assumption 3.1 be met and a, $c \in \mathbb{C}^{n} \backslash\{0\}$ satisfy $c_{k}^{\left(n_{k}\right)}=0$ or $a_{k}^{(1)}=0$. Then the solution given in Theorem 2.3 is not altered if we replace simultaneously $A$ by $\widetilde{A}$, and $a, c$ by $\widetilde{a}, \widetilde{c}$, where

a) $\widetilde{A}$ is the matrix obtained from $A$ by reducing the size of the Jordan block $A_{k}$ by one, more precisely by omitting in A the row/column which contains the last row/column of $A_{k}$,

b) $\widetilde{a}, \widetilde{c}$ are the vectors obtained from $a, c$ by deleting the entries

$$
\left\{\begin{array}{cl}
a_{k}^{\left(n_{k}\right)}, c_{k}^{\left(n_{k}\right)} & \text { for } c_{k}^{\left(n_{k}\right)}=0 \\
a_{k}^{(1)}, c_{k}^{(1)} & \text { for } a_{k}^{(1)}=0
\end{array}\right.
$$

Proof. We treat the case $c_{k}^{\left(n_{k}\right)}=0$, the other being symmetric.

Set $d(x, t)=\exp \left(A x-\mathrm{i} A^{2} t\right) c$. Note that $L_{0}(x, t)=d(x, t) a^{t r}$, and that $L(x, t)$ is the unique matrix satisfying the $(x, t)$-dependent Sylvester equation $A X(x, t)+$ $X(x, t) \bar{A}=d(x, t) \bar{a}^{t r}$.

Since $A$ is a block diagonal matrix with upper triangular matrices on the diagonal, the same holds for $\exp \left(A x-\mathrm{i} A^{2} t\right)$. More precisely, $\exp \left(A x-\mathrm{i} A^{2} t\right)=$ $\operatorname{diag}\left\{\exp \left(A_{j} x-\mathrm{i} A_{j}^{2} t\right) \mid j=1, \ldots, N\right\}$ and hence $d(x, t)=\left(d_{j}(x, t)\right)_{j}$ with $d_{j}(x, t)=$ $\exp \left(A_{j} x-\mathrm{i} A_{j}^{2} t\right) c_{j}$.

Since $\exp \left(A_{k} x-\mathrm{i} A_{k}^{2} t\right)$ is upper triangular, $c_{k}^{\left(n_{k}\right)}=0$ implies $d_{k}^{\left(n_{k}\right)}=0$, and we have

$$
d_{k}(x, t)=\left(\exp \left(\widetilde{A}_{k} x-\mathrm{i} \widetilde{A}_{k}^{2} t\right) \widetilde{c}_{k}, 0\right),
$$

where $\widetilde{A}_{k}$ is the block obtained from $A_{k}$ by reducing the dimension by one and $\widetilde{c}_{k}$ the vector obtained from $c_{k}$ by deleting the $n_{k}$ th entry. Consequently $L_{0}=$ $d(x, t) a^{t r}=\left(d_{i}(x, t) a_{j}^{t r}\right)_{i, j=1}^{N}$ has a zero-row, namely the $\left(n_{1}+\ldots+n_{k}\right)$ th row.

The fact that the same holds for $L$ can be seen from the factorization of the solution of the Sylvester equation given in Lemma 7.3. Now the assertion follows from expanding the determinants $P, p$. 
Remark 5.4. In the case $n_{k}=1$ the above procedure amounts to crossing out $A_{k}$, $a_{k}, c_{k}$ completely. If there is only one block left, this leads to the trivial solution.

\section{LINK TO ISM}

In this section we establish the link to Olmedilla'a derivation [15] of MPS's via ISM. This construction starts out from the data of principal parts

$$
r_{j}(k)=\frac{r_{j n_{j}}}{\left(k-k_{j}\right)^{n_{j}}}+\frac{r_{j n_{j}-1}}{\left(k-k_{j}\right)^{n_{j}-1}}+\ldots+\frac{r_{j 1}}{k-k_{j}}
$$

given at $N$ points $k_{1}, \ldots, k_{N}$ in the upper half plane $\mathbb{H}=\{\operatorname{Im}(k)>0\}$. Following [15], we associate to (6.1) the kernel

$$
\Omega(z ; t)=-2 \mathrm{i} \sum_{j=1}^{N} \operatorname{res}_{k=k_{j}}\left(r_{j}(k) e^{2 \mathrm{i} k z-4 \mathrm{i} k^{2} t}\right) .
$$

The main goal of this section is to solve the corresponding Gelfand-Levitan-Marchenko (GLM) equation

$$
K(x, y ; t)+\int_{0}^{\infty} \int_{0}^{\infty} K(x, s ; t) \Omega(x+s+z ; t) \overline{\Omega(x+y+z ; t)} d s d z=\overline{\Omega(x+y ; t)} .
$$

Step 1: Rewriting the kernel. Since the function $\ell(k)=e^{2 \mathrm{i} k z-4 \mathrm{i} k^{2} t}$ is holomorphic, its Taylor expansion together with 6.1) give

$$
\begin{aligned}
\operatorname{res}_{k=k_{j}}\left(r_{j}(k) \ell(k)\right) & =\sum_{\nu=1}^{n_{j}} \frac{r_{j \nu}}{(\nu-1) !} \frac{\partial^{\nu-1} \ell}{\partial k^{\nu-1}}\left(k_{j}\right) \\
& =\left(\ell_{1}\left(k_{j}\right), \ldots, \ell_{n_{j}}\left(k_{j}\right)\right) d_{j},
\end{aligned}
$$

where we have set $\ell_{\nu}(k)=\frac{1}{(\nu-1) !} \frac{\partial^{\nu-1} \ell}{\partial k^{\nu-1}}(k)$ and $d_{j}=\left(\begin{array}{c}r_{j 1} \\ \vdots \\ r_{j n_{j}}\end{array}\right)$.

Let $\Lambda_{j}=2 \mathrm{i} J_{j}$, where $J_{j}$ is the $n_{j} \times n_{j}$-Jordan block corresponding to the eigenvalue $k_{j}$. By Lemma 7.2 .

$$
e^{\Lambda_{j} z+\mathrm{i} \Lambda_{j}^{2} t}=\left(\begin{array}{ccc}
\ell_{1}\left(k_{j}\right) & & \ell_{n_{j}}\left(k_{j}\right) \\
0 & \ddots & \ell_{1}\left(k_{j}\right)
\end{array}\right) .
$$

Multiplying with the transposed of the first standard basis vector $e_{n_{j}}^{(1)}$ in $\mathbb{C}^{n_{j}}$ from the left gives us the first row of this matrix. Thus we find

$$
\operatorname{res}_{k=k_{j}}\left(r_{j}(k) \ell(k)\right)=\left(e_{n_{j}}^{(1)}\right)^{t r} e^{\Lambda_{j} z+\mathrm{i} \Lambda_{j}^{2} t} d_{j} .
$$

Let now $\Lambda=\operatorname{diag}\left\{\Lambda_{1}, \ldots, \Lambda_{N}\right\}$ be the block diagonal matrix with the $n_{j} \times n_{j}$-blocks $\Lambda_{j}$ on the diagonal. Then

$$
e^{\Lambda z+\mathrm{i} \Lambda^{2} t}=\operatorname{diag}\left\{e^{\Lambda_{j} z+\mathrm{i} \Lambda_{j}^{2} t} \mid j=1, \ldots, N\right\},
$$

and hence

$$
\begin{aligned}
\sum_{j=1}^{N} \operatorname{res}_{k=k_{j}}\left(r_{j}(k) \ell(k)\right) & =\sum_{j=1}^{N}\left(e_{n_{j}}^{(1)}\right)^{\operatorname{tr}} e^{\Lambda_{j} z+\mathrm{i} \Lambda_{j}^{2} t} d_{j} \\
& =f^{t r} e^{\Lambda z+\mathrm{i} \Lambda^{2} t} d
\end{aligned}
$$


for the vectors $f=\left(e_{n_{j}}^{(1)}\right)_{j=1}^{N}$ and $d=\left(d_{j}\right)_{j=1}^{N}$. Hence the kernel 6.2 can be written in the form

$$
\Omega(z ; t)=-2 \mathrm{i} f^{t r} e^{\Lambda z+\mathrm{i} \Lambda^{2} t} d .
$$

Observe that the spectrum of the matrix $\Lambda$ is contained in the left complex halfplane.

Step 2: Solving the GLM equation. Let $D$ be the unique solution of the Sylvester equation $\Lambda X+X \bar{\Lambda}=-2 \mathrm{i} d f^{t r}$ and set $G(x, t)=e^{\Lambda x+\mathrm{i} \Lambda^{2} t} D$. In this step we show that

$$
K(x, y ; t)=2 \mathrm{i} f^{t r}(I+\overline{G(x, t)} G(x, t))^{-1} e^{\bar{\Lambda}(x+y)-\mathrm{i} \bar{\Lambda}^{2} t} \bar{d}
$$

solves the GLM equation (6.3) with kernel (6.4) (see Appendix Cfor the invertibility of $I+\overline{G(x, t)} G(x, t)$ on $\left.\mathbb{R}^{2}\right)$.

To this end, we first observe that

$2 \mathrm{i} \int_{0}^{\infty} e^{\Lambda s} d f^{t r} e^{\bar{\Lambda} s} d s=-\int_{0}^{\infty} e^{\Lambda s}(\Lambda D+D \bar{\Lambda}) e^{\bar{\Lambda} s} d s=-\int_{0}^{\infty} \frac{d}{d s}\left(e^{\Lambda s} D e^{\bar{\Lambda} s}\right) d s=D$,

where we have used that the spectra of $\Lambda, \bar{\Lambda}$ are contained in the left complex half-plane. Since $f$ has real entries, we also have

$$
-2 \mathrm{i} \int_{0}^{\infty} e^{\bar{\Lambda} s} \bar{d} f^{t r} e^{\Lambda s} d s=\bar{D} .
$$

Abbreviating $M(x, t)=(I+\overline{G(x, t)} G(x, t))^{-1}$, we get

$$
\begin{aligned}
\int_{0}^{\infty} & K(x, s ; t) \Omega(x+s+z ; t) d s \\
= & -(2 \mathrm{i})^{2} \int_{0}^{\infty}\left(f^{\operatorname{tr}} M(x, t) e^{\bar{\Lambda}(x+s)-\mathrm{i} \bar{\Lambda}^{2} t} \bar{d}\right)\left(f^{\operatorname{tr}} e^{\Lambda(x+s+z)+\mathrm{i} \Lambda^{2} t} d\right) d s \\
= & 2 \mathrm{i} f^{t r} M(x, t)\left(-2 \mathrm{i} e^{\bar{\Lambda} x-\mathrm{i} \bar{\Lambda}^{2} t} \int_{0}^{\infty} e^{\bar{\Lambda} s} \bar{d} f^{t r} e^{\Lambda s} d s\right) e^{\Lambda(x+z)+\mathrm{i} \Lambda^{2} t} d \\
& \quad 2 \mathrm{i} f^{\operatorname{tr}} M(x, t) \overline{G(x, t)} e^{\Lambda(x+z)+\mathrm{i} \Lambda^{2} t} d .
\end{aligned}
$$

Now we can verify $(6.3)$ :

$$
\begin{aligned}
\int_{0}^{\infty} & \int_{0}^{\infty} K(x, s ; t) \Omega(x+s+z ; t) \overline{\Omega(x+y+z ; t)} d s d z \\
= & (2 \mathrm{i})^{2} \int_{0}^{\infty}\left(f^{\operatorname{tr}} M(x, t) \overline{G(x, t)} e^{\Lambda(x+z)+\mathrm{i} \Lambda^{2} t} d\right)\left(f^{t r} e^{\bar{\Lambda}(x+z+y)-\mathrm{i} \bar{\Lambda}^{2} t} \bar{d}\right) d z \\
= & 2 \mathrm{i} f^{t r} M(x, t) \overline{G(x, t)}\left(2 \mathrm{i} e^{\Lambda x+\mathrm{i} \Lambda^{2} t} \int_{0}^{\infty} e^{\Lambda z} d f^{t r} e^{\bar{\Lambda} z} d z\right) e^{\bar{\Lambda}(x+y)-\mathrm{i} \bar{\Lambda}^{2} t \bar{d}} \\
= & 2 \mathrm{i} f^{\operatorname{tr}}(M(x, t) \overline{G(x, t)} G(x, t)) e^{\bar{\Lambda}(x+y)-\mathrm{i} \bar{\Lambda}^{2} t} \bar{d} \\
= & 2 \mathrm{i} f^{t r}(I-M(x, t)) e^{\bar{\Lambda}(x+y)-\mathrm{i} \bar{\Lambda}^{2} t} \bar{d} \\
= & \overline{\Omega(x+y ; t)}-K(x, y ; t) .
\end{aligned}
$$

Step 3: Conclusions. From the formalism of the ISM, the corresponding potential $q$ is obtained via

$$
\begin{aligned}
q(x, t) & =-K(x, 0 ; t) \\
& =-2 \mathrm{i} f^{t r}(I+\overline{G(x, t)} G(x, t))^{-1} e^{\bar{\Lambda} x-\mathrm{i} \bar{\Lambda}^{2} t} \bar{d} .
\end{aligned}
$$


Using the trace representation (2.6), it is easy to check that these potentials are included in the solution class of Theorem 2.3 for

$$
A=\bar{\Lambda}, \quad a=-2 \mathrm{i} f, \quad c=\bar{d} .
$$

Let us summarize: The MPS's considered in [15] are derived as solutions of 6.3 with kernels as in (6.2). In all these cases we have solved $(6.3)$ by functions $K$ whose associated potentials are included in the solution class of Theorem 2.3. Because of known uniqueness properties of the GLM equation (see [3. page 24]), the results in [15] on asymptotic behavior of MPS's (only MPS's consisting of one wave packet with at most three waves are treated in [15) are special cases of Theorem 3.3 . Note also that the above reasoning complements the derivation of explicit expressions for solutions in [15, where the GLM equation is only solved for $N=1$.

\section{Algebraic Simplification of Data}

In the following proposition MPS's are rewritten in a form suitable for asymptotic analysis.

Proposition 7.1. The determinants $P, p$ in the formula of the solution given in Theorem 2.3 can be expressed as

$$
\begin{aligned}
p(x, t) & =\operatorname{det}\left(I+\left(\begin{array}{cc}
M & 0 \\
0 & \bar{M}
\end{array}\right)\left(\begin{array}{cc}
0 & -T \\
\bar{T} & 0
\end{array}\right)\right), \\
P(x, t) & =\operatorname{det}\left(I+\left(\begin{array}{cc}
M & 0 \\
0 & \bar{M}
\end{array}\right)\left(\begin{array}{cc}
-f f^{t r} & -T \\
\bar{T} & 0
\end{array}\right)\right),
\end{aligned}
$$

where

a) $T=\left(T_{i j}\right)_{i, j=1}^{N}$ with

$$
T_{i j}=\left((-1)^{\mu+\nu}\left(\begin{array}{c}
\mu+\nu-2 \\
\mu-1
\end{array}\right)\left(\frac{1}{\alpha_{i}+\bar{\alpha}_{j}}\right)^{\mu+\nu-1}\right)_{\substack{\mu=1, \ldots, n_{i} \\
\nu=1, \ldots, n_{j}}},
$$

b) $f \in \mathbb{C}^{n}$ denotes the vector $f=\left(e_{n_{j}}^{(1)}\right)_{j=1}^{N}$ consisting of the first standard basis vectors $e_{n_{j}}^{(1)} \in \mathbb{C}^{n_{j}}$ for $j=1, \ldots, N$, and

c) $M=\operatorname{diag}\left\{M_{j} \mid j=1, \ldots, N\right\}$ with

$$
M_{j}=\left(\begin{array}{cc}
m_{j}^{(1)} & m_{j}^{\left(n_{j}\right)} \\
m_{j}^{\left(n_{j}\right)} & 0
\end{array}\right) \in \mathcal{M}_{n_{j}, n_{j}}(\mathbb{C}),
$$

the entries given by

$$
\begin{aligned}
& m_{j}^{(\mu)}=m_{j}^{(\mu)}(x, t)=\sum_{\kappa=1}^{n_{j}-(\mu-1)} b_{j}^{(\mu-1+\kappa)} \frac{1}{(\kappa-1) !} \frac{\partial^{\kappa-1}}{\partial \alpha_{j}^{\kappa-1}} \exp \left(\alpha_{j} x-\mathrm{i} \alpha_{j}^{2} t\right) \\
& \text { with constants } b_{j}^{(\mu)}=\sum_{\kappa=1}^{n_{j}-(\mu-1)} a_{j}^{(\kappa)} c_{j}^{(\mu-1+\kappa)} \text { for } \mu=1, \ldots, n_{j} .
\end{aligned}
$$

We start with the following observation. 
Lemma 7.2. Let $J$ be a Jordan block of size $m \times m$ with eigenvalue $\alpha$, and let $p(\alpha)$ be a polynomial. Then

$$
e^{p(J)}=\Gamma_{r}\left(\left(\ell_{\mu}\right)_{\mu=1}^{m}\right)=\left(\begin{array}{ccc}
\ell_{1}(\alpha) & & \ell_{m}(\alpha) \\
& \ddots & \\
0 & & \ell_{1}(\alpha)
\end{array}\right)
$$

is an ( $\alpha$-dependent) upper right band matrix with

$$
\ell_{\mu}(\alpha)=\frac{1}{(\mu-1) !} \frac{\partial^{\mu-1}}{\partial \alpha^{\mu-1}} e^{p(\alpha)} .
$$

Proof. Note that for $f=f(\alpha), g=g(\alpha)$ the Leibniz rule implies

$\Gamma_{r}\left(\left(\frac{1}{(\mu-1) !} \frac{\partial^{\mu-1} f}{\partial \alpha^{\mu-1}}\right)_{\mu=1}^{m}\right) \Gamma_{r}\left(\left(\frac{1}{(\mu-1) !} \frac{\partial^{\mu-1} g}{\partial \alpha^{\mu-1}}\right)_{\mu=1}^{m}\right)=\Gamma_{r}\left(\left(\frac{1}{(\mu-1) !} \frac{\partial^{\mu-1}(f g)}{\partial \alpha^{\mu-1}}\right)_{\mu=1}^{m}\right)$

Because of this and the functional equation of the exponential function, it is enough to show the lemma for a monomial $p(\alpha)=c \alpha^{k}$. To this end one first verifies by induction that

$$
\left.J^{\kappa}=\Gamma_{r}\left(\left(\begin{array}{c}
\kappa \\
\mu-1
\end{array}\right) \alpha^{\kappa-\mu+1}\right)_{\mu=1}^{m}\right)=\Gamma_{r}\left(\left(\frac{1}{(\mu-1) !} \frac{\partial^{\mu-1}}{\partial \alpha^{\mu-1}} \alpha^{\kappa}\right)_{\mu=1}^{m}\right)
$$

for $\kappa \geq 0$. Hence,

$$
\begin{aligned}
e^{c J^{k}} & =\sum_{\kappa \geq 0} \frac{c^{\kappa}}{\kappa !} J^{k \kappa} \\
& =\sum_{\kappa \geq 0} \frac{c^{\kappa}}{\kappa !} \Gamma_{r}\left(\left(\frac{1}{(\mu-1) !} \frac{\partial^{\mu-1}}{\partial \alpha^{\mu-1}} \alpha^{k \kappa}\right)_{\mu=1}^{m}\right) \\
& =\Gamma_{r}\left(\left(\frac{1}{(\mu-1) !} \frac{\partial^{\mu-1}}{\partial \alpha^{\mu-1}} \sum_{\kappa \geq 0} \frac{c^{\kappa}}{\kappa !} \alpha^{k \kappa}\right)_{\mu=1}^{m}\right) \\
& =\Gamma_{r}\left(\left(\frac{1}{(\mu-1) !} \frac{\partial^{\mu-1}}{\partial \alpha^{\mu-1}} e^{c \alpha^{k}}\right)_{\mu=1}^{m}\right) .
\end{aligned}
$$

which completes the proof.

Lemma 7.3. Let Assumption 3.1 be satisfied, and let $C$ be the unique solution of the Sylvester equation $A X+X \bar{A}=c \bar{a}^{\text {tr }}$. Then

$$
C=\left(\Gamma_{l}\left(c_{i}\right) T_{i j} \Gamma_{r}\left(\bar{a}_{j}\right)\right)_{i, j=1}^{N},
$$

where the $T_{i j}$ are as defined in Theorem 2.3, and $\Gamma_{l}\left(c_{i}\right), \Gamma_{r}\left(\bar{a}_{j}\right)$ denote the upper left and right band matrices given by

$$
\Gamma_{l}\left(c_{i}\right)=\left(\begin{array}{ccc}
c_{i}^{(1)} & & c_{i}^{\left(n_{i}\right)} \\
c_{i}^{\left(n_{i}\right)} & \ddots & 0
\end{array}\right), \quad \Gamma_{r}\left(\bar{a}_{j}\right)=\left(\begin{array}{ccc}
\bar{a}_{j}^{(1)} & & \bar{a}_{j}^{\left(n_{j}\right)} \\
& \ddots & \\
0 & & \bar{a}_{j}^{(1)}
\end{array}\right) .
$$

Proof. Let us first discuss the case of two Jordan blocks $A, B$ of sizes $m \times m, n \times n$ with eigenvalues $\alpha, \beta$, respectively. We claim that the matrix

$$
T=\left((-1)^{\mu+\nu}\left(\begin{array}{c}
\mu+\nu-2 \\
\mu-1
\end{array}\right)\left(\frac{1}{\alpha+\beta}\right)^{\mu+\nu-1}\right)_{\substack{\mu=1, \ldots, m \\
\nu=1, \ldots, n}}
$$

satisfies

$$
A^{t r} T+T B=E_{m n}:=\left(\delta_{\mu 1} \delta_{\nu 1}\right)_{\substack{\mu=1, \ldots, m \\ \nu=1, \ldots, n}}^{\substack{t, \ldots \\ \text {. }}} .
$$


Indeed, from

$$
\left(A^{\operatorname{tr} T}\right)_{\mu \nu}=\left\{\begin{array}{cl}
\alpha t_{1 \nu}, & \mu=1, \\
\alpha t_{\mu \nu}+t_{(\mu-1) \nu}, & \mu>1,
\end{array} \quad(T B)_{\mu \nu}=\left\{\begin{array}{cc}
\beta t_{\mu 1}, & \nu=1, \\
\beta t_{\mu \nu}+t_{\mu(\nu-1)}, & \nu>1,
\end{array}\right.\right.
$$

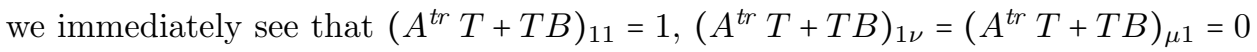
for $\mu, \nu>1$. Furthermore, for $\mu, \nu>1$,

$$
\begin{aligned}
& \left(A^{\operatorname{tr}} T+T B\right)_{\mu \nu}=(\alpha+\beta) t_{\mu \nu}+t_{(\mu-1) \nu}+t_{\mu(\nu-1)} \\
& \quad=(-1)^{\mu+\nu}\left[\left(\begin{array}{c}
\mu+\nu-2 \\
\mu-1
\end{array}\right)-\left(\begin{array}{c}
\mu+\nu-3 \\
\mu-1
\end{array}\right)-\left(\begin{array}{c}
\mu+\nu-3 \\
\mu-2
\end{array}\right)\right]\left(\frac{1}{\alpha+\beta}\right)^{\mu+\nu-2} \\
& \quad=0 .
\end{aligned}
$$

This proves the claim.

Next we show that, for $c \in \mathbb{C}^{m}, b \in \mathbb{C}^{n}$, the matrix $S=\Gamma_{l}(c) T \Gamma_{r}(b)$ satisfies $A S+S B=c b^{t r}$. To this end we observe $\left[\Gamma_{r}(b), B\right]=0$ and $A \Gamma_{l}(c)=\Gamma_{l}(c) A^{t r}$. Thus,

$$
\begin{aligned}
A S+S B & =A\left(\Gamma_{l}(c) T \Gamma_{r}(b)\right)+\left(\Gamma_{l}(c) T \Gamma_{r}(b)\right) B \\
& =\Gamma_{l}(c)\left(A^{t r} T+T B\right) \Gamma_{r}(b)=\Gamma_{l}(c) E_{m n} \Gamma_{r}(b)=c b^{t r} .
\end{aligned}
$$

From this the assertion follows using Assumption 3.1 .

Proof of Proposition 7.1. We focus on $P$, which is the more involved case. The arguments for $p$ are similar but easier.

Set $F=F(x, t)=\exp \left(A x-\mathrm{i} A^{2} t\right)$. Then $F=\operatorname{diag}\left\{F_{j} \mid j=1, \ldots, N\right\}$ where, according to Lemma 7.2 .

$$
F_{j}=\left(\begin{array}{ccc}
\ell_{j}^{(1)} & & \ell_{j}^{\left(n_{j}\right)} \\
& \ddots & \\
0 & & \ell_{j}^{(1)}
\end{array}\right)
$$

with $\ell_{j}^{(\mu)}=\ell_{j}^{(\mu)}(x, t)=\left.\frac{1}{(\mu-1) !} \frac{\partial^{\mu-1}}{\partial \alpha^{\mu-1}}\right|_{\alpha=\alpha_{j}} e^{\alpha x-\mathrm{i} \alpha^{2} t}$.

By Lemma 7.3, we have $C=\Gamma_{c} T \bar{\Gamma}_{a}$ for $\Gamma_{c}=\operatorname{diag}\left\{\Gamma_{l}\left(c_{j}\right) \mid j=1, \ldots, N\right\}$ and $\Gamma_{a}=\operatorname{diag}\left\{\Gamma_{r}\left(a_{j}\right) \mid j=1, \ldots, N\right\}$. Furthermore, one checks

$$
\begin{aligned}
c a^{t r} & =\left(c_{i} a_{j}^{t r}\right)_{i, j=1}^{N}=\left(\Gamma_{l}\left(c_{i}\right) E_{n_{i} n_{j}} \Gamma_{r}\left(a_{j}\right)\right)_{i, j=1}^{N}=\Gamma_{c}\left(e_{n_{i}}^{(1)}\left(e_{n_{j}}^{(1)}\right)^{t r}\right)_{i, j=1}^{N} \Gamma_{a} \\
& =\Gamma_{c}\left(f f^{t r}\right) \Gamma_{a} .
\end{aligned}
$$

Hence,

$$
\begin{aligned}
P & =\operatorname{det}\left(I+\left(\begin{array}{cc}
-F\left(c a^{t r}\right) & -F C \\
\overline{F C} & 0
\end{array}\right)\right) \\
& =\operatorname{det}\left(I+\left(\begin{array}{cc}
F \Gamma_{c} & 0 \\
0 & \overline{F \Gamma}_{c}
\end{array}\right)\left(\begin{array}{cc}
-f f^{t r} & -T \\
\bar{T} & 0
\end{array}\right)\left(\begin{array}{cc}
\Gamma_{a} & 0 \\
0 & \bar{\Gamma}_{a}
\end{array}\right)\right) \\
& =\operatorname{det}\left(I+\left(\begin{array}{cc}
M & 0 \\
0 & \bar{M}
\end{array}\right)\left(\begin{array}{cc}
-f f^{t r} & -T \\
\bar{T} & 0
\end{array}\right)\right),
\end{aligned}
$$

where $M=\Gamma_{a} F \Gamma_{c}$. Hence $M=\operatorname{diag}\left\{M_{j} \mid j=1, \ldots, N\right\}$ with $M_{j}=\Gamma_{r}\left(a_{j}\right) F_{j} \Gamma_{l}\left(c_{j}\right)=$ $F_{j}\left(\Gamma_{r}\left(a_{j}\right) \Gamma_{l}\left(c_{j}\right)\right)=F_{j} \Gamma_{l}\left(b_{j}\right)=\Gamma_{l}\left(\left(m_{j}^{(\mu)}\right)_{\mu=1}^{n_{j}}\right)$ as claimed in c). 


\section{Collisions of Wave PaCKets}

In this section we show that MPS's corresponding to $N$ Jordan blocks asymptotically are superpositions of $N$ single wave packets.

We only consider $t \rightarrow-\infty$, since the case $t \rightarrow+\infty$ is completely symmetric. In particular we may assume $t<0$ in the sequel.

Recall that the velocity of the $k$ th wave packet is $v_{k}=-2 \operatorname{Im}\left(\alpha_{k}\right)$. Without loss of generality we may assume $v_{1}>\ldots>v_{N}$. Then $\Lambda_{k}^{-}=\{1, \ldots, k-1\}$.

Proposition 8.1. For the solution in Theorem 2.3, it holds

$$
q(x, t) \approx \sum_{k=1}^{N} q^{(k)}(x, t) \text { for } t \approx-\infty,
$$

where $q^{(k)}=1-P^{(k)} / p^{(k)}$ with

$$
\begin{aligned}
& P^{(k)}=\operatorname{det}\left(\left(\begin{array}{cc}
J^{(k)} & 0 \\
0 & J^{(k)}
\end{array}\right)+\left(\begin{array}{cc}
M^{(k)} & 0 \\
0 & \bar{M}^{(k)}
\end{array}\right)\left(\begin{array}{cc}
-f^{(k)}\left(f^{(k)}\right)^{t r} & -T^{(k)} \\
\bar{T}^{(k)} & 0
\end{array}\right)\right) \\
& p^{(k)}=\operatorname{det}\left(\left(\begin{array}{cc}
J^{(k)} & 0 \\
0 & J^{(k)}
\end{array}\right)+\left(\begin{array}{cc}
M^{(k)} & 0 \\
0 & \bar{M}^{(k)}
\end{array}\right)\left(\begin{array}{cc}
0 & -T^{(k)} \\
\bar{T}^{(k)} & 0
\end{array}\right)\right) .
\end{aligned}
$$

The blocks of the above matrices are of size $\left(n^{(k)}+n_{k}\right) \times\left(n^{(k)}+n_{k}\right)$ where $n^{(k)}=$ $\sum_{j=1}^{k-1} n_{j}$ and given by

$$
\begin{array}{ll}
J^{(k)}=\left(\begin{array}{cc}
0_{n^{(k)}} & 0 \\
0 & I_{n_{k}}
\end{array}\right), & M^{(k)}=\left(\begin{array}{cc}
I_{n^{(k)}} & 0 \\
0 & M_{k}
\end{array}\right), \\
T^{(k)}=\left(T_{i j}\right)_{i, j=1}^{k}, & f^{(k)}=\left(e_{n_{j}}^{(1)}\right)_{j=1}^{k}
\end{array}
$$

with $T_{i j}, e_{n_{j}}^{(1)}$, and $M_{k}$ as defined in Proposition 7.1 .

For the proof we need the following elementary perturbation lemma. For a matrix $S$, we denote by $|S|$ the maximum of the moduli of its entries.

Lemma 8.2. Let $\delta, \delta_{0} \geq 0$ and $c, c_{0} \geq 1$. Furthermore, let $S(t), S_{0}(t) \in \mathcal{M}_{m, m}(\mathbb{C})$ be defined for $t \leq t_{0}$ and satisfy $\left|S_{0}(t)\right| \leq c_{0} e^{\delta_{0}|t|},\left|S(t)-S_{0}(t)\right| \leq c e^{-\delta|t|}$. Then there is a constant $\gamma>0$, only depending on $m$, such that

$$
\left|\operatorname{det}(S(t))-\operatorname{det}\left(S_{0}(t)\right)\right| \leq \gamma\left(c c_{0}\right)^{m} e^{-\left(\delta-m \delta_{0}\right)|t|}
$$

for all $t \leq t_{0}$.

Proof. Let $\operatorname{Perm}(m)$ be the set of all permutations of $\{1, \ldots, m\}$. Set $S_{1}=S-S_{0}$. Then, for $S_{0}=\left(s_{0, i j}\right)_{i, j=1}^{m}, S_{1}=\left(s_{1, i j}\right)_{i, j=1}^{m}$ we have

$$
\begin{aligned}
\operatorname{det}(S) & =\operatorname{det}\left(S_{0}+S_{1}\right)=\sum_{\pi \in \operatorname{Perm}(m)} \operatorname{sgn}(\pi) \prod_{j=1}^{m}\left(s_{0, j \pi(j)}+s_{1, j \pi(j)}\right) \\
& =\operatorname{det}\left(S_{0}\right)+\sum_{\pi \in \operatorname{Perm}(m)} \operatorname{sgn}(\pi) \sum_{\mu=1}^{m} \sum_{i_{1}<\ldots<i_{\mu}}\left[\prod_{\kappa=1}^{\mu} s_{1, i_{\kappa} \pi\left(i_{\kappa}\right)} \prod_{j \notin\left\{i_{1}, \ldots, i_{\mu}\right\}} s_{0, j \pi(j)}\right] .
\end{aligned}
$$

Since

$$
\left|\prod_{\kappa=1}^{\mu} s_{1, i_{\kappa} \pi\left(i_{\kappa}\right)} \prod_{j \neq i_{1}, \ldots, i_{\mu}} s_{0, j \pi(j)}\right| \leq c^{m} e^{-\mu \delta|t|} c_{0}^{m-\mu} e^{(m-\mu) \delta_{0}|t|} \leq\left(c c_{0}\right)^{m} e^{-\left(\delta-m \delta_{0}\right)|t|}
$$

we obtain 8.1 for $\gamma=m !\left(2^{m}-1\right)$. 
Proof of Proposition 8.1. Recall $\operatorname{Re}\left(\alpha_{j}\right)>0 \forall j$ by Assumption a) of Theorem 3.3 .

Let $\delta_{k}=\frac{1}{1+\lambda_{k}} \min _{j=k \pm 1}\left|v_{j}-v_{k}\right|$ for some $\lambda_{k}>\max \left(1, \frac{8 n \operatorname{Re}\left(\alpha_{k}\right)}{\min _{j=k \pm 1} \operatorname{Re}\left(\alpha_{j}\right)}\right)$.

Fix $k$ and consider

$$
\mathcal{I}_{k}(t)=\left(v_{k} t-\delta_{k}|t|, v_{k} t+\delta_{k}|t|\right) \text {. }
$$

This interval has the center $v_{k} t$, and its diameter grows linearly with $|t|$. Furthermore,

$$
\mathcal{C}_{k}=\bigcup_{t<0} \mathcal{I}_{k}(t)
$$

is a cone with vertex in the origin.

Actually it is not essential that the cones have vertices in one point (the

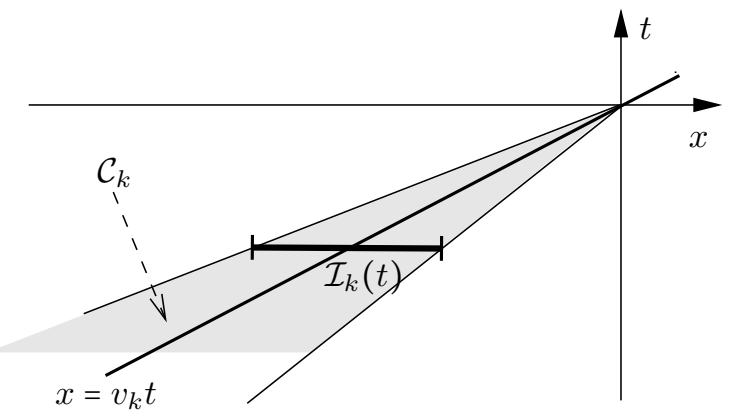
origin). Our choice is technically convenient since it ensures that the cones are disjoint.

The proof is organized as follows. We may assume that the solution is in reduced form, i.e. $q=1-P / p$ with $P, p$ as given in Proposition 7.1. We show that the following properties hold asymptotically:

Step 1: $q \approx q^{(k)}$ in $\mathcal{C}_{k}$, i.e. the only contribution comes from the $k$ th wave packet,

Step 2: $q^{(k)} \approx 0$ outside $\mathcal{C}_{k}$,
Step 3: $q \approx 0$ outside $\bigcup_{k=1}^{N} \mathcal{C}_{k}$.

Step 1. Let us start with some elementary estimates, which hold uniformly on $x \in \mathcal{I}_{k}(t)$ for $|t|$ sufficiently large.

Since, for $\ell_{k}=e^{\alpha_{k} x-\mathrm{i} \alpha_{k}^{2} t}$, we have $\left|\ell_{k}\right|=e^{\operatorname{Re}\left(\alpha_{k}\right)\left(x-v_{k} t\right)} \leq e^{\operatorname{Re}\left(\alpha_{k}\right) \delta_{k}|t|}$ for all $t$, we first obtain that, for all $j$,

$$
\left|M_{k} T_{k j}\right|,\left|M_{k} e_{n_{k}}^{(1)}\left(e_{n_{j}}^{(1)}\right)^{t r}\right|<e^{2 \operatorname{Re}\left(\alpha_{k}\right) \delta_{k}|t|}
$$

provided that $|t|$ is sufficiently large.

For $i>k$, we have $v_{i}<v_{k}$. Hence $\left(1+\lambda_{k}\right) \delta_{k}=\min _{j=k \pm 1}\left|v_{j}-v_{k}\right| \leq v_{k}-v_{i}$ by definition of $\delta_{k}$. As a consequence,

$$
\begin{aligned}
x-v_{i} t & =\left(x-v_{k} t\right)+\left(v_{k}-v_{i}\right) t=\left(x-v_{k} t\right)-\left(v_{k}-v_{i}\right)|t| \\
& <\delta_{k}|t|-\left(1+\lambda_{k}\right) \delta_{k}|t|=-\lambda_{k} \delta_{k}|t| .
\end{aligned}
$$

Thus, $\left|\ell_{i}\right|<e^{-\operatorname{Re}\left(\alpha_{i}\right) \lambda_{k} \delta_{k}|t|}<e^{-\beta \lambda_{k} \delta_{k}|t|}$ for all $t$, where we have set $\beta=\min _{j} \operatorname{Re}\left(\alpha_{j}\right)$. This shows, for $i>k$ and all $j$,

$$
\left|M_{i} T_{i j}\right|,\left|M_{i} e_{n_{i}}^{(1)}\left(e_{n_{j}}^{(1)}\right)^{t r}\right|<e^{-\frac{1}{2} \beta \lambda_{k} \delta_{k}|t|}
$$

for $|t|$ sufficiently large.

For $i<k$ one analogously obtains $\left|\ell_{i}^{-1}\right|<e^{-\beta \lambda_{k} \delta_{k}|t|}$ for all $t$. Note that $M_{i}^{-1}$ is of the same structure as $M_{i}$ with some different constants and the exponential function $\ell_{i}^{-1}$. Hence

$$
\left|M_{i}^{-1}\right|<e^{-\frac{1}{2} \beta \lambda_{k} \delta_{k}|t|}
$$


for $|t|$ sufficiently large.

Set $D=\operatorname{diag}\left\{M_{1}^{-1}, \ldots, M_{k-1}^{-1}, I_{n_{k}}, \ldots, I_{n_{N}}\right\}$. Then the solution $q=1-P / p$ is not changed upon replacing $P, p$ by $\widehat{P}, \widehat{p}$, where

$$
\widehat{P}=\operatorname{det}\left(\begin{array}{cc}
D & 0 \\
0 & \bar{D}
\end{array}\right) P, \quad \widehat{p}=\operatorname{det}\left(\begin{array}{cc}
D & 0 \\
0 & \bar{D}
\end{array}\right) p .
$$

Furthermore,

$$
\begin{aligned}
\widehat{P} & =\operatorname{det}\left(\left(\begin{array}{cc}
D & 0 \\
0 & \bar{D}
\end{array}\right)\left(I+\left(\begin{array}{cc}
M & 0 \\
0 & \bar{M}
\end{array}\right)\left(\begin{array}{cc}
-f f^{t r} & -T \\
\bar{T} & 0
\end{array}\right)\right)\right) \\
& =\operatorname{det}(S), \quad \text { where } S=\left(\begin{array}{cc}
D-U & -V \\
\bar{V} & \bar{D}
\end{array}\right)
\end{aligned}
$$

with $U=D M f f^{t r}$ and $V=D M T$. In particular, $U, V$ have the following block structure

$$
U_{i j}=\left\{\begin{array}{rl}
e_{n_{i}}^{(1)}\left(e_{n_{j}}^{(1)}\right)^{t r}, & i<k, \\
M_{i} e_{n_{i}}^{(1)}\left(e_{n_{j}}^{(1)}\right)^{t r}, & i \geq k,
\end{array} \quad V_{i j}=\left\{\begin{aligned}
T_{i j}, & i<k, \\
M_{i} T_{i j}, & i \geq k .
\end{aligned}\right.\right.
$$

Consider next

$$
\widehat{P}_{0}=\operatorname{det}\left(S_{0}\right) \quad \text { for } S_{0}=\left(\begin{array}{cc}
J-U_{0} & -V_{0} \\
\bar{V}_{0} & J
\end{array}\right),
$$

where $J=\operatorname{diag}\left\{0, \ldots, 0, I_{n_{k}}, \ldots, I_{n_{N}}\right\}$ and the blocks of $U_{0}, V_{0}$ are given by

$$
U_{0, i j}=\left\{\begin{array}{rl}
e_{n_{i}}^{(1)}\left(e_{n_{j}}^{(1)}\right)^{t r}, & i<k, \\
M_{k} e_{n_{k}}^{(1)}\left(e_{n_{j}}^{(1)}\right)^{t r}, & i=k, \\
0, & i>k,
\end{array} \quad V_{0, i j}=\left\{\begin{aligned}
T_{i j}, & i<k, \\
M_{k} T_{k j}, & i=k, \\
0, & i>k
\end{aligned}\right.\right.
$$

Observe that $S_{0}$ is obtained from $S$ by replacing the blocks $M_{i}, \bar{M}_{i}, i>k$, and the blocks $M_{i}^{-1}, \bar{M}_{i}^{-1}, i<k$, by zero blocks. From $\left.8.2,8.3\right),(8.4)$, we see that Lemma 8.2 can be applied with $\delta=\frac{1}{2} \beta \lambda_{k} \delta_{k}, \delta_{0}=2 \operatorname{Re}\left(\alpha_{k}\right) \delta_{k}$ and $c_{0}=c=1$. Furthermore, $\gamma:=\delta-2 n \delta_{0}=2 \delta_{k}\left(\lambda_{k} \beta-8 n \operatorname{Re}\left(\alpha_{k}\right)\right)>0$ by the choice of $\lambda_{k}$. Hence,

$$
\sup _{x \in \mathcal{I}_{k}(t)}\left|\widehat{P}(x, t)-\widehat{P}_{0}(x, t)\right| \lesssim e^{-\gamma|t|}
$$

for $|t|$ sufficiently large.

Finally, inspection of the determinant $\widehat{P}_{0}$ shows that by deleting all blocks corresponding to the indices $k+1, \ldots, N$ (which does not change the determinant) one obtains $P^{(k)}$. As a result,

$$
\sup _{x \in \mathcal{I}_{k}(t)}\left|\widehat{P}(x, t)-P^{(k)}(x, t)\right| \longrightarrow 0 \quad \text { as } t \rightarrow-\infty
$$

A similar, but easier argument also shows $\sup _{x \in \mathcal{I}_{k}(t)}\left|\widehat{p}(x, t)-p^{(k)}(x, t)\right| \rightarrow 0$.

Observe that $\widehat{p} \neq 0$ on $\mathbb{R}^{2}$ since $q$ is a regular solution (see Appendix C). Hence the above convergence implies $p^{(k)} \neq 0$ on $\mathcal{I}_{k}(t)$ for sufficiently large $|t|$, and we can transfer the convergence from $\widehat{P}, \widehat{p}$ to $\widehat{q}=1-\widehat{P} / \widehat{p}$. In summary, we have shown

$$
\mathbb{1}_{\mathcal{C}_{k}} q \approx \mathbb{1}_{\mathcal{C}_{k}} q^{(k)} \text { for } t \approx-\infty,
$$

where $\mathbb{1}_{\mathcal{C}_{k}}$ is the characteristic function of $\mathcal{C}_{k}$, and Step $\mathbf{1}$ is complete. 
Step 2. To discuss the behavior of the $k$ th wave packet $q^{(k)}$ outside of $\mathcal{I}_{k}(t)$, we distinguish two cases:

Let first $x \in \mathcal{I}_{k}^{-}(t)=\left(-\infty, v_{k} t-\delta_{k}|t|\right]$. In this case the entries of $M_{k}$ decay exponentially motivating to compare the determinants of the matrices $S_{0}$ and $S$ given by

$$
S_{0}=\left(\begin{array}{cc}
J^{(k)}-U_{0}^{(k)} & -V_{0}^{(k)} \\
\overline{V_{0}^{(k)}} & J^{(k)}
\end{array}\right), \quad S=\left(\begin{array}{cc}
J^{(k)}-U^{(k)} & -V^{(k)} \\
\overline{V^{(k)}} & J^{(k)}
\end{array}\right),
$$

with $U_{0}^{(k)}, V_{0}^{(k)}, U^{(k)}, V^{(k)}$ consisting of the blocks

$$
U_{0, i j}^{(k)}=\left\{\begin{array}{rl}
e_{n_{i}}^{(1)}\left(e_{n_{j}}^{(1)}\right)^{t r}, & i<k, \\
0, & i=k,
\end{array} \quad V_{0, i j}^{(k)}=\left\{\begin{aligned}
T_{i j}, & i<k, \\
0, & i=k,
\end{aligned}\right.\right.
$$

and

$$
U_{i j}^{(k)}=\left\{\begin{array}{rl}
e_{n_{i}}^{(1)}\left(e_{n_{j}}^{(1)}\right)^{t r}, & i<k, \\
M_{k} e_{n_{k}}^{(1)}\left(e_{n_{j}}^{(1)}\right)^{t r}, & i=k,
\end{array} \quad V_{i j}^{(k)}=\left\{\begin{aligned}
T_{i j}, & i<k \\
M_{k} T_{k j}, & i=k .
\end{aligned}\right.\right.
$$

In particular, $U^{(k)}=M^{(k)} f^{(k)}\left(f^{(k)}\right)^{t r}$ and $V^{(k)}=M^{(k)} T^{(k)}$, which implies that $\operatorname{det}(S)=P^{(k)}$. Note also that $S_{0}$ is obtained from $S$ by replacing appearances of $M_{k}, \bar{M}_{k}$ by a zero blocks, and that $S_{0}$ is a constant matrix. Applying Lemma 8.2 with $\delta=\frac{1}{2} \operatorname{Re}\left(\alpha_{k}\right) \delta_{k}, \delta_{0}=0$, and $c=1, c_{0}=\left|S_{0}\right|$, we obtain

$$
\sup _{x \in \mathcal{I}_{\bar{k}}^{-}(t)}\left|P^{(k)}(x, t)-\operatorname{det}\left(S_{0}\right)\right| \longrightarrow 0 \quad \text { as } t \rightarrow-\infty .
$$

Furthermore,

$$
\operatorname{det}\left(S_{0}\right)=\operatorname{det}\left(\begin{array}{cc}
-f^{-}\left(f^{-}\right)^{t r} & -T^{-} \\
\frac{\left(T^{-}\right)}{0} & 0
\end{array}\right)
$$

where $T^{-}=\left(T_{i j}\right)_{i, j=1}^{k-1}, f^{-}=\left(f_{j}\right)_{j=1}^{k-1}$.

Cauchy-type determinants as $\operatorname{det}\left(T^{-}\right)$have been evaluated in 24]. In particular, [24, Theorem 4.1] implies $\operatorname{det}\left(T^{-}\right) \neq 0$. Observe also $\overline{\left(T^{-}\right)}=\left(T^{-}\right)^{t r}$. Hence $1^{1}$ $\operatorname{det}\left(S_{0}\right)=\operatorname{det}\left(T^{-}\right)^{2}$.

A similar, but easier argument also shows $\sup _{x \in \mathcal{I}_{k}^{-}(t)}\left|p^{(k)}(x, t)-\operatorname{det}\left(T^{-}\right)^{2}\right| \rightarrow 0$, and because of $\operatorname{det}\left(T^{-}\right) \neq 0$, convergence can be transferred from $P^{(k)}, p^{(k)}$ to $q^{(k)}=1-P^{(k)} / p^{(k)}$.

Let now $x \in \mathcal{I}_{k}^{+}(t)=\left[v_{k} t+\delta_{k}|t|, \infty\right)$. In this case we first replace $P^{(k)}, p^{(k)}$ by $\widehat{P}^{(k)}, \widehat{p}^{(k)}$, where

$$
\widehat{P}^{(k)}=P^{(k)} / \operatorname{det}\left(\begin{array}{cc}
M^{(k)} & 0 \\
0 & \overline{M^{(k)}}
\end{array}\right), \quad \widehat{p}^{(k)}=p^{(k)} / \operatorname{det}\left(\begin{array}{cc}
M^{(k)} & 0 \\
0 & \overline{M^{(k)}}
\end{array}\right),
$$

${ }^{1}$ For square matrices $A, B, C$ of the same size, $B$ invertible,

$$
\operatorname{det}\left(\begin{array}{cc}
A & -B \\
C & 0
\end{array}\right)=\operatorname{det}\left(\left(\begin{array}{cc}
A & -B \\
C & 0
\end{array}\right)\left(\begin{array}{cc}
I & 0 \\
B^{-1} A & I
\end{array}\right)\right)=\operatorname{det}\left(\begin{array}{cc}
0 & -B \\
C & 0
\end{array}\right)=\operatorname{det}(B) \operatorname{det}(C)
$$


which does not change $q^{(k)}=1-P^{(k)} / p^{(k)}$. Setting $D^{(k)}=\operatorname{diag}\left\{0, \ldots, 0, M_{k}^{-1}\right\}$, we then have achieved

$$
\widehat{P}^{(k)}=\operatorname{det}\left(\begin{array}{cc}
D^{(k)}-\frac{f^{(k)}}{T^{(k)}}\left(f^{(k)}\right)^{\operatorname{tr}} & \frac{-T^{(k)}}{D^{(k)}}
\end{array}\right) .
$$

Now we can proceed similarly as in the first case of Step 2. Using first Lemma 8.2 to replace $D^{(k)}$ by the zero matrix 0 , and then simplifying the resulting determinant, one shows

$$
\sup _{x \in \mathcal{I}_{k}^{+}(t)}\left|\widehat{P}^{(k)}(x, t)-\operatorname{det}\left(T^{(k)}\right)^{2}\right| \longrightarrow 0, \quad \sup _{x \in \mathcal{I}_{k}^{+}(t)}\left|\widehat{p}^{(k)}(x, t)-\operatorname{det}\left(T^{(k)}\right)^{2}\right| \longrightarrow 0
$$

as $t \rightarrow-\infty$, and convergence can be transferred to $q^{(k)}=1-P^{(k)} / p^{(k)}$ since $\operatorname{det}\left(T^{(k)}\right) \neq 0$. Summing up, we have shown that

$$
\left(1-\mathbb{1}_{\mathcal{C}_{k}}\right) q^{(k)} \approx 0 \text { for } t \approx-\infty,
$$

completing Step 2.

Step 3. To discuss the behavior of $q$ outside $\cup_{k=1}^{N} \mathcal{C}_{k}$, we distinguish three cases.

Consider first the interval

$$
\mathcal{J}_{k}(t)=\left[v_{k} t+\delta_{k}|t|, v_{k+1} t-\delta_{k+1}|t|\right]
$$

for $k<N$, which covers the gap between the two neighboring intervals $\mathcal{I}_{k}(t)$ and $\mathcal{I}_{k+1}(t)$.

Note that $\mathcal{J}_{k}(t)$ has positive length since $\delta_{k}<\frac{1}{2} \min _{j \neq k \pm 1}\left|v_{j}-v_{k}\right|$ for all $k$.

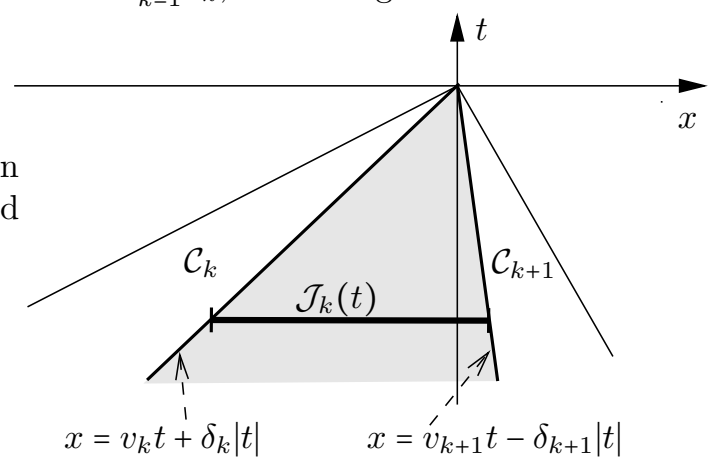

For $x \in \mathcal{J}_{k}(t)$ one has

$$
\begin{aligned}
x-v_{i} t & \leq\left(v_{k+1}-v_{i}\right) t-\delta_{k+1}|t| \\
& =-\left(\left|v_{k+1}-v_{i}\right|+\delta_{k+1}\right)|t| \leq-\delta_{k+1}|t|, \quad i>k, \\
x-v_{i} t & \geq\left(v_{k}-v_{i}\right) t+\delta_{k}|t| \\
& =\left(\left|v_{i}-v_{k}\right|+\delta_{k}\right)|t| \geq \delta_{k}|t|, \quad i \leq k,
\end{aligned}
$$

which yields the estimates $\left|\ell_{i}\right| \leq e^{-\beta \min \left(\delta_{k}, \delta_{k+1}\right)|t|}$ for $i>k$ and $\left|\ell_{i}^{-1}\right| \leq e^{-\beta \min \left(\delta_{k}, \delta_{k+1}\right)|t|}$ for $i \leq k$. Hence, setting $\beta_{k}=\frac{1}{2} \beta \min \left(\delta_{k}, \delta_{k+1}\right)$, we find

$$
\left|M_{i}^{-1}\right| \leq e^{-\beta_{k}|t|} \text { for } i \leq k \text { and }\left|M_{i}\right| \leq e^{-\beta_{k}|t|} \text { for } i>k .
$$

for sufficiently large $|t|$.

Replacing $P, p$ by $\widehat{P}, \widehat{p}$, where

$$
\widehat{P}=\operatorname{det}\left(\begin{array}{cc}
D & 0 \\
0 & \bar{D}
\end{array}\right) P, \quad \widehat{p}=\operatorname{det}\left(\begin{array}{cc}
D & 0 \\
0 & \bar{D}
\end{array}\right) p,
$$

and $D=\operatorname{diag}\left\{M_{1}^{-1}, \ldots, M_{k}^{-1}, I_{n_{k+1}}, \ldots, I_{n_{N}}\right\}$, which does not change $q=1-P / p$, one gets

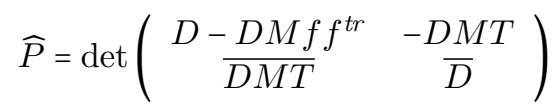


with $D M=\operatorname{diag}\left\{I_{n_{1}}, \ldots, I_{n_{k}}, M_{k+1}, \ldots, M_{N}\right\}$. Similarly as is Step 2 one can now use 8.5 and Lemma 8.2 to replace $D$ by $J=\operatorname{diag}\left\{0, \ldots, 0, I_{n_{k+1}}, \ldots, I_{n_{N}}\right\}$ and $D M$ by $I-J$. After simplification of the resulting determinants, this results in

$$
\sup _{x \in \mathcal{J}_{k}(t)}\left|\widehat{P}(x, t)-\operatorname{det}(\widehat{T})^{2}\right| \longrightarrow 0, \quad \sup _{x \in \mathcal{J}_{k}(t)}\left|\widehat{p}(x, t)-\operatorname{det}(\widehat{T})^{2}\right| \longrightarrow 0
$$

as $t \rightarrow-\infty$, where $\widehat{T}=\left(T_{i j}\right)_{i, j=1}^{k}$.

Referring to [24, Theorem 4.1] for $\operatorname{det}(\widehat{T}) \neq 0$, convergence can be transferred from $\widehat{P}, \widehat{p}$ to $\widehat{q}=1-\widehat{P} / \widehat{p}$.

Second we consider $x \in \mathcal{J}_{\min }(t)=\left(-\infty, v_{1} t-\delta_{1}|t|\right]$. In this case, $x-v_{i} t \leq-\delta_{1}|t|$ for all $i$, hence Lemma 8.2 can be applied directly to replace $M$ by 0 , which gives

$$
\sup _{x \in \mathcal{J}_{\max }(t)}|P(x, t)-1| \longrightarrow 0, \quad \sup _{x \in \mathcal{J}_{\max }(t)}|p(x, t)-1| \longrightarrow 0,
$$

as $t \rightarrow-\infty$, and we are done.

Finally we consider $x \in \mathcal{J}_{\max }(t)=\left[v_{N} t+\delta_{N}|t|, \infty\right)$. Here $x-v_{i} t \geq \delta_{n}|t|$ for all $i$. Replacing $P, p$ by $\widehat{P}, \widehat{p}$ with

$$
\widehat{P}=\operatorname{det}\left(\begin{array}{cc}
M^{-1} & 0 \\
0 & \bar{M}^{-1}
\end{array}\right) P, \quad \widehat{p}=\operatorname{det}\left(\begin{array}{cc}
M^{-1} & 0 \\
0 & \bar{M}^{-1}
\end{array}\right) p,
$$

which does not change $q=1-P / p$, one finds

$$
\widehat{P}=\operatorname{det}\left(\begin{array}{cc}
M^{-1}-f f^{t r} & -T \\
\bar{T} & \bar{M}^{-1}
\end{array}\right)
$$

Using Lemma 8.2 to replace $M^{-1}$ by 0 , one arrives at

$$
\sup _{x \in \mathcal{J}_{\min }(t)}\left|\widehat{P}(x, t)-\operatorname{det}(T)^{2}\right| \longrightarrow 0, \quad \sup _{x \in \mathcal{J}_{\min }(t)}\left|\widehat{p}(x, t)-\operatorname{det}(T)^{2}\right| \longrightarrow 0,
$$

as $t \rightarrow-\infty$. Since $\operatorname{det}(T) \neq 0$, see [24, Theorem 4.1], convergence can be transferred. Summing up,

$$
\left(1-\sum_{k=1}^{N} \mathbb{1}_{\mathcal{C}_{k}}\right) q \approx 0 \text { for } t \approx-\infty,
$$

which completes Step 3.

\section{Conclusion.}

$$
\begin{aligned}
q & \approx \sum_{k=1}^{N} \mathbb{1}_{\mathcal{C}_{k}} q+\left(1-\sum_{k=1}^{N} \mathbb{1}_{\mathcal{C}_{k}}\right) q^{\text {Steps }} \underset{\approx}{\mathbf{1}, 3} \sum_{k=1}^{N} \mathbb{1}_{\mathcal{C}_{k}} q^{(k)}+0 \\
\underset{\sim \operatorname{Step}}{\stackrel{2}{*}} & \sum_{k=1}^{N} \mathbb{1}_{\mathcal{C}_{k}} q^{(k)}+\sum_{k=1}^{N}\left(1-\mathbb{1}_{\mathcal{C}_{k}}\right) q^{(k)} \\
& =\sum_{k=1}^{N} q^{(k)}, \quad t \approx-\infty .
\end{aligned}
$$

This completes the proof of Proposition 8.1 


\section{Collisions of the solitons Within A WAVE PACKet}

In this section turn to the fine analysis of the $k$ th wave packet $q^{(k)}$ by studying what happens if we deviate from the path $x=v_{k} t$ logarithmically.

Proposition 9.1. For $\rho \in \mathbb{R}$, define $\gamma_{\rho}(t)=v_{k} t+\rho \log |t| / \operatorname{Re}\left(\alpha_{k}\right)$. Then, for the determinants $P^{(k)}, p^{(k)}$ given in Proposition 8.1, it holds

$$
\begin{aligned}
p^{(k)}\left(\gamma_{\rho}(t), t\right) & =C^{2}\left(\sum_{\kappa=0}^{n_{k}} C_{\kappa \kappa} F_{\kappa}(t) \overline{F_{\kappa}(t)}|t|^{2 \rho \kappa} t^{-2 \kappa^{2}+2 n_{k} \kappa}\right)\left[1+\mathcal{O}\left(\frac{\log |t|}{|t|}\right)\right], \\
P^{(k)}\left(\gamma_{\rho}(t), t\right) & =C^{2}\left(\sum_{\kappa=0}^{n_{k}} C_{\kappa \kappa} F_{\kappa}(t) \overline{F_{\kappa}(t)}|t|^{2 \rho \kappa} t^{-2 \kappa^{2}+2 n_{k} \kappa}+\right. \\
& \left.+\sum_{\kappa=0}^{n_{k}-1}(-1)^{\kappa+1} C_{(\kappa+1) \kappa} F_{\kappa+1}(t) \overline{F_{\kappa}(t)}|t|^{2 \rho \kappa+\rho} t^{-2 \kappa^{2}+2\left(n_{k}-1\right) \kappa+\left(n_{k}-1\right)}\right)\left[1+\mathcal{O}\left(\frac{\log |t|}{|t|}\right)\right]
\end{aligned}
$$

with

$$
\begin{aligned}
C & =\operatorname{det}\left(T_{i j}\right)_{i j=1}^{k-1}, \\
C_{\kappa \lambda} & =\left[\frac{1}{\alpha_{k}+\bar{\alpha}_{k}}\right]^{2 \kappa \lambda} \prod_{j=1}^{k-1}\left[\frac{\alpha_{j}-\alpha_{k}}{\bar{\alpha}_{j}+\alpha_{k}}\right]^{2 \kappa n_{j}}\left[\frac{\bar{\alpha}_{j}-\bar{\alpha}_{k}}{\alpha_{j}+\bar{\alpha}_{k}}\right]^{2 \lambda n_{j}}, \\
F_{\kappa}(t) & =\frac{\prod_{\kappa^{\prime}=1}^{\kappa-1} \kappa^{\prime} !}{\prod_{\kappa^{\prime}=1}^{\kappa}\left(n_{k}-\kappa^{\prime}\right) !}\left(d_{k}^{n_{k}-\kappa} b_{k}^{\left(n_{k}\right)} e^{\mathrm{i} \Upsilon_{k}\left(\gamma_{\rho}(t), t\right)}\right)^{\kappa}
\end{aligned}
$$

where $d_{k}=-2 \mathrm{iRe}\left(\alpha_{k}\right)$ and $\Upsilon_{k}(x, t)=\operatorname{Im}\left(\alpha_{k} x-\mathrm{i} \alpha_{k}^{2} t\right)$.

Note that the parameter $\rho$ controls the logarithmic deviation. It enters in the asymptotic via the terms $|t|^{2 \kappa \rho}, \kappa=0, \ldots, n_{k}$, and $\Upsilon_{k}\left(\gamma_{\rho}(t), t\right)$.

The main part of the proof consists in expanding the determinants $P^{(k)}, p^{(k)}$. To this end we need some more notation. Consider the matrix

$$
S=\left(\begin{array}{cc}
U & V \\
W & Z
\end{array}\right)
$$

where $U$ has the block structure

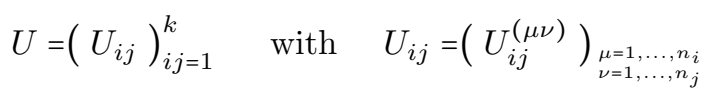

and $V, W, Z$ are built analogously. For index tuples $J=\left(\sigma_{1}, \ldots, \sigma_{\kappa}\right), J^{\prime}=$ $\left(\sigma_{1}^{\prime}, \ldots, \sigma_{\kappa}^{\prime}\right), K=\left(\tau_{1}, \ldots, \tau_{\lambda}\right), K^{\prime}=\left(\tau_{1}^{\prime}, \ldots, \tau_{\lambda}^{\prime}\right)$ with $\sigma_{\kappa^{\prime}}, \sigma_{\kappa^{\prime}}^{\prime}, \tau_{\lambda^{\prime}}, \tau_{\lambda^{\prime}}^{\prime} \in\left\{1, \ldots, n_{k}\right\}$ for $1 \leq \kappa^{\prime} \leq \kappa, 1 \leq \lambda^{\prime} \leq \lambda$, we define the matrix $S\left[J, K ; J^{\prime}, K^{\prime}\right]$ by

$$
S\left[J, K ; J^{\prime}, K^{\prime}\right]=\left(\begin{array}{cc}
U\left[J, J^{\prime}\right] & V\left[J, K^{\prime}\right] \\
W\left[K, J^{\prime}\right] & Z\left[K, K^{\prime}\right]
\end{array}\right) .
$$

Here $U\left[J, J^{\prime}\right]$ denotes the matrix with the blocks $U\left[J, J^{\prime}\right]_{i j}, i, j=1, \ldots, k$, where

(1) $U\left[J, J^{\prime}\right]_{i j}=U_{i j}$ if $i<k$ and $j<k$,

(2) $U\left[J, J^{\prime}\right]_{k j}$ consists of the rows nr. $\sigma_{1}, \ldots, \sigma_{\kappa}$ from $U_{k j}$ for $j<k$,

(3) $U\left[J, J^{\prime}\right]_{i k}$ consists of the columns nr. $\sigma_{1}^{\prime}, \ldots, \sigma_{\kappa}^{\prime}$ from $U_{i k}$ for $i<k$,

(4) $U\left[J, J^{\prime}\right]_{k k}$ is obtained from $U_{k k}$ by first selecting the rows nr. $\sigma_{1}, \ldots, \sigma_{\kappa}$ and then, from the resulting matrix, the columns $\mathrm{nr} . \sigma_{1}^{\prime}, \ldots, \sigma_{\kappa}^{\prime}$ (of course we get the same result by first selecting columns). 


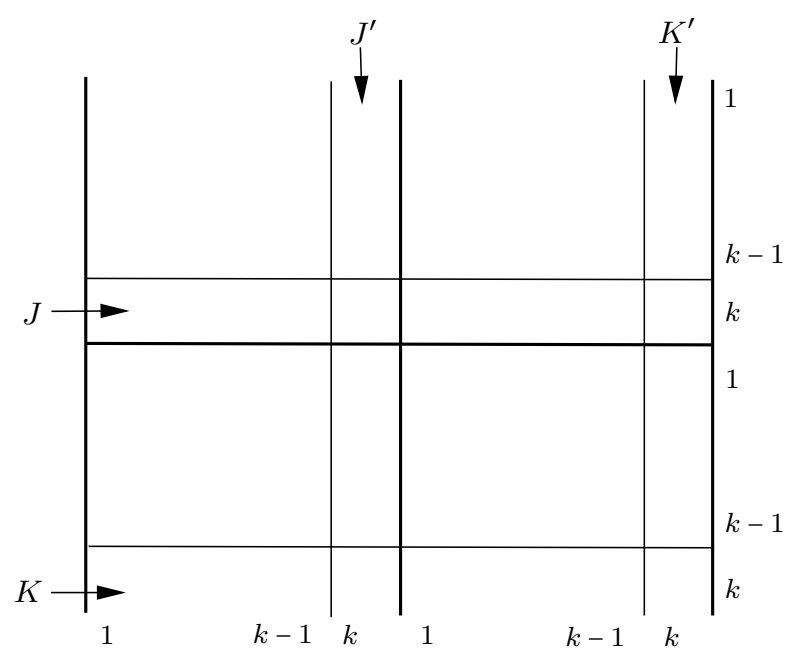

Figure 6 . To illustrate $S\left[J, K ; J^{\prime}, K^{\prime}\right]$, we indicate the position of the blocks affected by the modifications.

Note that any ordering of the index tuples $J, J^{\prime}$ and even repetitions of indices are admitted. $V\left[J, K^{\prime}\right], W\left[K, J^{\prime}\right], Z\left[K, K^{\prime}\right]$ are defined accordingly.

By $|J|$ we denote the length of the index tuple $J$, in the case at hand $|J|=\kappa$. Note that also the trivial case $|J|=0$ is admitted, where all rows of the respective blocks are eliminated.

Finally, with respect to the expansion in mind, we define $\widehat{I}=\operatorname{diag}\left\{J^{(k)}, J^{(k)}\right\}$ for $J^{(k)}=\operatorname{diag}\left\{0, \ldots, 0, I_{n_{k}}\right\}$. Routine arguments give:

Lemma 9.2. The following expansion rule holds:

$$
\operatorname{det}(\widehat{I}+S)=\sum_{\kappa=0}^{n_{k}} \sum_{\lambda=0}^{n_{k}} \sum_{|J|=\kappa}{ }^{\prime} \sum_{|K|=\lambda}{ }^{\prime} \operatorname{det}(S[J, K ; J, K]),
$$

where the inner sums are taken over all index tuples $J, K$ from $\left\{1, \ldots, n_{k}\right\}$ and the prime means that only tuples with strictly increasing entries are admitted.

Recall that the cases $\kappa=0, \lambda=0$ correspond to the appearance of empty index sets.

To determine the non-vanishing contributions in the expansion, we need the following elementary lemma.

Lemma 9.3. Let $B \in \mathcal{M}_{m, n}(\mathbb{C}), C \in \mathcal{M}_{n, m}(\mathbb{C})$ be arbitrary, and $b, c \in \mathbb{C}^{m}$. If either $m<n$ or $n+1<m$, then

$$
\operatorname{det}\left(\begin{array}{cc}
c b^{t r} & B \\
C & 0
\end{array}\right)=0
$$

Proof. If $m<n$ holds, the last $n$ columns are linearly dependent. If $n+1<m$, the space $U=\left\{d \in \mathbb{C}^{m} \mid C d=0\right\}$ has dimension at least 2. The different ways of combining the zero vector from the columns of $c b^{\text {tr }}$ are parameterized by the space $V=\left\{d \in \mathbb{C}^{m} \mid b^{\text {tr }} d=0\right\}$, which has codimension at most 1 in $\mathbb{C}^{m}$. Hence $U \cap V \neq\{0\}$ and the first $m$ columns are linearly dependent. 
The next lemmata (which have already been proved in 21]) are needed for the explicit evaluation of certain determinants.

Lemma 9.4. For all $\gamma \in \mathbb{C}$,

$$
\operatorname{det}\left(\prod_{\nu^{\prime}=1}^{\nu-1}\left(\gamma-\mu-\nu^{\prime}+1\right)\right)_{\mu, \nu=1}^{m}=(-1)^{\frac{m(m+3)}{2}} \prod_{\mu=1}^{m-1} \mu !
$$

Proof. Multiplying the $\nu$ th column by $(\gamma-m-\nu+1)$ and subtracting it from the $(\nu+1)$ th column for $\nu=m-1, \ldots, 1$ (in the indicated order) gives

$$
\begin{aligned}
\Delta & =\operatorname{det}\left(\prod_{\nu^{\prime}=1}^{\nu-1}\left(\gamma-\mu-\nu^{\prime}+1\right)\right)_{\mu, \nu=1}^{m} \\
& =\operatorname{det}\left(1,(m-\mu) \prod_{\nu^{\prime}=1}^{\nu-2}\left(\gamma-\mu-\nu^{\prime}+1\right)\right)_{\substack{\mu \geq 1 \\
\nu>1}} .
\end{aligned}
$$

Next we expand the determinant with respect to the $m$ th row, which is zero except for the first entry. Extracting then the factor $(m-\mu)$, which is common to the $\mu$ th row for $\mu=1, \ldots, m-1$, results in

$$
\Delta=(-1)^{m+1}(m-1) ! \operatorname{det}\left(\prod_{\nu^{\prime}=1}^{\nu-1}\left(\gamma-\mu-\nu^{\prime}+1\right)\right)_{\mu, \nu=1}^{m-1},
$$

and the assertion follows by induction.

Lemma 9.5. Let $\gamma \in \mathbb{N}$ with $\gamma \geq m$. Then, for the $m \times m$ matrix $F=\left(f_{\mu \nu}\right)_{\mu, \nu=1}^{m}$ given by

$$
f_{\mu \nu}=\left\{\begin{array}{cc}
\frac{1}{(\gamma-\mu-\nu+1) !}, & \mu+\nu \leq \gamma+1, \\
0, & \mu+\nu>\gamma+1,
\end{array}\right.
$$

it holds

$$
\operatorname{det}(F)=(-1)^{\frac{m(m+3)}{2}} \frac{\prod_{\mu=1}^{m-1} \mu !}{\prod_{\mu=1}^{m}(\gamma-\mu) !}
$$

Proof. The identity

$$
(\gamma-\mu) ! f_{\mu \nu}=\frac{(\gamma-\mu) !}{(\gamma-\mu-\nu+1) !}=\prod_{\nu^{\prime}=1}^{\nu-1}\left(\gamma-\mu-\nu^{\prime}+1\right)
$$

holds for $\mu+\nu \leq \gamma+1$. It also holds for $\mu+\nu>\gamma+1$ since in the product on the right the factor corresponding to $\nu^{\prime}=\gamma-\mu+1<\nu$ vanishes. Hence,

$$
\operatorname{det}(F)=\operatorname{det}\left(\frac{1}{(\gamma-\mu) !} \prod_{\nu^{\prime}=1}^{\nu-1}\left(\gamma-\mu-\nu^{\prime}+1\right)\right)_{\mu, \nu=1}^{m},
$$

and, after extracting the factors $1 /(\gamma-\mu)$ ! common to the $\mu$ th row, the assertion follows from Lemma 9.4 .

Proof of Proposition 9.1. First we provide estimates for the entries $m_{k}^{(\mu)}$ of $M_{k}$, see Proposition 7.1 for their definition.

For $\ell(\alpha ; x, t)=e^{\alpha x-\mathrm{i} \alpha^{2} t}$ and $\mu \geq 0$, we define the polynomials $q_{\mu}(\alpha ; x, t)$ by $q_{\mu}=\ell^{-1} \partial^{\mu} \ell / \partial \alpha^{\mu}$. Note that they satisfy the recursion relation $q_{\mu+1}=q_{\mu} q_{1}+\partial q_{\mu} / \partial \alpha$, where $q_{0}=1, q_{1}=x-2 \mathrm{i} \alpha t$. 
Observe that $\operatorname{Re}\left(\alpha_{k}\right)\left(x-v_{k} t\right)=\rho \log |t|$ for $x=\gamma_{\rho}(t)$. Hence, $\ell\left(\alpha_{k} ; \gamma_{\rho}(t), t\right)=$ $|t|^{\rho} e^{\mathrm{i} \Upsilon_{k}\left(\gamma_{\rho}(t), t\right)}$, and an inductive argument shows

$$
q_{\mu}\left(\alpha_{k} ; \gamma_{\rho}(t), t\right)=\left(d_{k} t\right)^{\mu}+\mathcal{O}\left(|t|^{\mu-1} \log |t|\right), \quad \frac{\partial q_{\mu}}{\partial \alpha}\left(\alpha_{k} ; \gamma_{\rho}(t), t\right)=\mathcal{O}\left(|t|^{\mu}\right) .
$$

As a result,

$$
m_{k}^{(\mu)}\left(\gamma_{\rho}(t), t\right)=\frac{d_{k}^{n_{k}-\mu} b_{k}^{\left(n_{k}\right)} e^{\mathrm{i} \Upsilon_{k}\left(\gamma_{\rho}(t), t\right)}}{\left(n_{k}-\mu\right) !} t^{n_{k}-\mu}|t|^{\rho}\left[1+\mathcal{O}\left(\frac{\log |t|}{|t|}\right)\right] .
$$

In the proof, we focus on the treatment of $P^{(k)}$, which is the far more involved case. Write $P^{(k)}=\operatorname{det}(\widehat{I}+S)$ with

$$
S=\left(\begin{array}{cc}
-M^{(k)} f^{(k)}\left(f^{(k)}\right)^{t r} & -M^{(k)} T^{(k)} \\
M^{(k)}\left(T^{(k)}\right)^{t r} & 0
\end{array}\right),
$$

where we have used $\overline{T^{(k)}}=\left(T^{(k)}\right)^{t r}$.

Applying the expansion rule in Lemma 9.2 , we obtain

$$
\begin{aligned}
P^{(k)=} \sum_{\kappa=0}^{n_{k}-1} & \sum_{|J|=\kappa+1}{ }^{\prime} \sum_{|K|=\kappa}{ }^{\prime} \operatorname{det}(S[J, K ; J, K])+ \\
& +\sum_{\kappa=0}^{n_{k}} \sum_{|J|=\kappa}{ }^{\prime} \sum_{|K|=\kappa}{ }^{\prime} \operatorname{det}(S[J, K ; J, K]),
\end{aligned}
$$

where we have used Lemma 9.3 to see that the principal minors $\operatorname{det}(S[J, K ; J, K])$ only contribute for $|K|=|J|-1$ and $|K|=|J|$. Our next aim is to evaluate those principal minors.

Recall that $M^{(k)} T^{(k)}$ has the blocks $T_{i j}$ for $i<k$ and $M_{k} T_{k j}$ for $i=k$, where, from the upper left band structure of $M_{k}$, one finds

$$
M_{k} T_{k j}=\left(\sum_{\kappa=1}^{n_{k}-(\mu-1)} t_{k j}^{(\kappa \nu)} m_{k}^{(\mu+\kappa-1)}\right)_{\substack{\mu=1, \ldots, n_{k} \\ \nu=1, \ldots, n_{j}}} .
$$

Note also that $M_{k} e_{n_{k}}^{(1)}\left(e_{n_{j}}^{(1)}\right)^{t r}$ has a similar structure. Using linearity of the determinant with respect to rows, we observe for $J=\left(\sigma_{1}, \ldots, \sigma_{\kappa}\right), K=\left(\tau_{1}, \ldots, \tau_{\lambda}\right)$ with strictly increasing indices, we have

$$
\begin{aligned}
& \operatorname{det}(S[J, K ; J, K])=\sum_{\widehat{\sigma}_{1}=1}^{n_{k}-\sigma_{1}+1} m_{k}^{\left(\sigma_{1}+\widehat{\sigma}_{1}-1\right)} \cdots \sum_{\widehat{\sigma}_{\kappa}=1}^{n_{k}-\sigma_{\kappa}+1} m_{k}^{\left(\sigma_{\kappa}+\widehat{\sigma}_{\kappa}-1\right)} \\
& \sum_{\tau_{1}=1}^{n_{k}-\tau_{1}+1} \bar{m}_{k}^{\left(\tau_{1}+\widehat{\tau}_{1}-1\right)} \cdots \sum_{\widehat{\tau}_{\lambda}=1}^{n_{k}-\tau_{\lambda}+1} \bar{m}_{k}^{\left(\tau_{\lambda}+\widehat{\tau}_{\lambda}-1\right)} \operatorname{det}(R[\widehat{J}, \widehat{K} ; J, K])
\end{aligned}
$$

with $\widehat{J}=\left(\widehat{\sigma}_{1}, \ldots, \widehat{\sigma}_{\kappa}\right), \widehat{K}=\left(\widehat{\tau}_{1}, \ldots, \widehat{\tau}_{\lambda}\right)$, and

$$
R=\left(\begin{array}{cc}
-f^{(k)}\left(f^{(k)}\right)^{t r} & -T^{(k)} \\
\left(T^{(k)}\right)^{t r} & 0
\end{array}\right) .
$$

Since $\operatorname{det}(R[\widehat{J}, \widehat{K} ; J, K])=0$ if $\widehat{J}$ or $\widehat{K}$ contains two coinciding indices, $\widehat{J}$ and $\widehat{K}$ can be assumed to contain pairwise different indices. 
We now look for those terms in 9.3 which are of leading order in $t$. From 9.1) we find that these correspond to the powers

$$
\begin{aligned}
\sum_{\kappa^{\prime}=1}^{\kappa}(\rho & \left.+n_{k}+1-\left(\sigma_{\kappa^{\prime}}+\widehat{\sigma}_{\kappa^{\prime}}\right)\right)+\sum_{\lambda^{\prime}=1}^{\lambda}\left(\rho+n_{k}+1-\left(\tau_{\lambda^{\prime}}+\widehat{\tau}_{\lambda^{\prime}}\right)\right) \\
= & (\kappa+\lambda)\left(\rho+n_{k}+1\right)-\sum_{\kappa^{\prime}=1}^{\kappa}\left(\sigma_{\kappa^{\prime}}+\widehat{\sigma}_{\kappa^{\prime}}\right)-\sum_{\lambda^{\prime}=1}^{\lambda}\left(\tau_{\lambda^{\prime}}+\widehat{\tau}_{\lambda^{\prime}}\right),
\end{aligned}
$$

with the constraint $\sigma_{\kappa^{\prime}}+\widehat{\sigma}_{\kappa^{\prime}}, \tau_{\lambda^{\prime}}+\widehat{\tau}_{\lambda^{\prime}} \leq n_{k}+1$ for $1 \leq \kappa^{\prime} \leq \kappa, 1 \leq \lambda^{\prime} \leq \lambda$, due to the limitations of the summation in 9.3 .

Let Perm $(\kappa)$ denote the group of permutations of $\{1, \ldots, \kappa\}$ and Perm' $(\kappa)$ the subset of those of the permutations $\pi \in \operatorname{Perm}(\kappa)$ which satisfy $\kappa^{\prime}+\pi\left(\kappa^{\prime}\right) \leq n_{k}+1$ for all $\kappa^{\prime}=1, \ldots, \kappa$. Observe that $\sum_{\kappa^{\prime}=1}^{\kappa} \sigma_{\kappa^{\prime}}$ is minimal iff $\sigma_{\kappa^{\prime}}=\kappa^{\prime}$ since the $\sigma_{\kappa^{\prime}}$ are strictly increasing, and since the $\widehat{\sigma}_{\kappa^{\prime}}$ are pairwise different, $\sum_{\kappa^{\prime}=1}^{\kappa} \widehat{\sigma}_{\kappa^{\prime}}$ is minimal iff $\widehat{\sigma}_{\kappa^{\prime}}=\pi\left(\kappa^{\prime}\right)$ with $\pi \in \operatorname{Perm}^{\prime}(\kappa)$.

Hence the expression in (9.4) attains its maximum precisely for $J=J_{0}, K=K_{0}$, where $J_{0}=(1, \ldots, \kappa), K_{0}=(1, \ldots, \lambda)$, and $\widehat{J}=\pi\left(J_{0}\right)=(\pi(1), \ldots, \pi(\kappa))$ with $\pi \in \operatorname{Perm}^{\prime}(\kappa), \widehat{K}=\chi\left(K_{0}\right)=(\chi(1), \ldots, \chi(\lambda))$ with $\chi \in \operatorname{Perm}^{\prime}(\lambda)$. To sum up,

$$
\operatorname{det}(S[J, K ; J, K])=H_{\kappa \lambda}(t)\left[1+\mathcal{O}\left(\frac{1}{|t|}\right)\right]
$$

with

$$
\begin{array}{r}
H_{\kappa \lambda}(t)=\sum_{\pi \in \operatorname{Perm}^{\prime}(\kappa)} \sum_{\chi \in \operatorname{Perm}^{\prime}(\lambda)} \prod_{\kappa^{\prime}=1}^{\kappa} m_{k}^{\left(\kappa^{\prime}+\pi\left(\kappa^{\prime}\right)-1\right)} \prod_{\lambda^{\prime}=1}^{\lambda} \bar{m}_{k}^{\left(\lambda^{\prime}+\chi\left(\lambda^{\prime}\right)-1\right)} \\
\operatorname{det}\left(R\left[\pi\left(J_{0}\right), \chi\left(K_{0}\right) ; J_{0}, K_{0}\right]\right) .
\end{array}
$$

Using 9.1 and $\sum_{\kappa^{\prime}=1}^{\kappa}\left(n_{k}-\left(\kappa^{\prime}+\pi\left(\kappa^{\prime}\right)-1\right)\right)=\left(n_{k}+1\right) \kappa-2 \sum_{\kappa^{\prime}=1}^{\kappa} \kappa^{\prime}=\left(n_{k}-\kappa\right) \kappa$, we find that

$$
\prod_{\kappa^{\prime}=1}^{\kappa} m_{k}^{\left(\kappa^{\prime}+\pi\left(\kappa^{\prime}\right)-1\right)}=\frac{G_{\kappa}(t) t^{\left(n_{k}-\kappa\right) \kappa}|t|^{\kappa \rho}}{\prod_{\kappa^{\prime}=1}^{\kappa}\left(n_{k}+1-\left(\kappa^{\prime}+\pi\left(\kappa^{\prime}\right)\right)\right) !} \quad\left[1+\mathcal{O}\left(\frac{\log |t|}{|t|}\right)\right]
$$

with

$$
G_{\kappa}(t)=\left(d_{k}^{n_{k}-\kappa} b_{k}^{\left(n_{k}\right)} e^{\mathrm{i} \Upsilon_{k}\left(\gamma_{\rho}(t), t\right)}\right)^{\kappa}
$$

(note that $G_{\kappa}(t)$ does not contribute to the growth in $t$ since $\left|G_{\kappa}(t)\right|$ is constant). Inserting this into 9.6 , we get

$$
H_{\kappa \lambda}(t)=D_{\kappa \lambda} G_{\kappa}(t) \overline{G_{\lambda}(t)} \quad|t|^{(\kappa+\lambda) \rho} t^{\kappa\left(n_{k}-\kappa\right)+\lambda\left(n_{k}-\lambda\right)} \quad\left[1+\mathcal{O}\left(\frac{\log |t|}{|t|}\right)\right],
$$

where

$$
\begin{aligned}
D_{\kappa \lambda}= & \sum_{\substack{\pi \in \operatorname{Perm}^{\prime}(\kappa) \\
\chi \in \operatorname{Perm}^{\prime}(\lambda)}} \prod_{\kappa^{\prime}=1}^{\kappa} \frac{1}{\left(n_{k}+1-\left(\kappa^{\prime}+\pi\left(\kappa^{\prime}\right)\right)\right) !} \prod_{\lambda^{\prime}=1}^{\lambda} \frac{1}{\left(n_{k}+1-\left(\lambda^{\prime}+\chi\left(\lambda^{\prime}\right)\right)\right) !} \\
& \operatorname{det}\left(R\left[\pi\left(J_{0}\right), \chi\left(K_{0}\right) ; J_{0}, K_{0}\right]\right) .
\end{aligned}
$$


We can drop the restriction on the permutations if we pass to the quantities

$$
f_{\mu \nu}=\left\{\begin{array}{cc}
\frac{1}{\left(n_{k}+1-(\mu+\nu)\right) !}, & \mu+\nu \leq n_{k}+1, \\
0, & \mu+\nu>n_{k}+1 .
\end{array} .\right.
$$

Moreover, $\operatorname{det}\left(R\left[\pi\left(J_{0}\right), \chi\left(K_{0}\right) ; J_{0}, K_{0}\right]\right)=\operatorname{sgn}(\pi) \operatorname{sgn}(\chi) \operatorname{det}\left(R\left[J_{0}, K_{0} ; J_{0}, K_{0}\right]\right)$ by reordering rows. Hence,

$$
\begin{aligned}
D_{\kappa \lambda}= & \sum_{\pi \in \operatorname{Perm}(\kappa)} \operatorname{sgn}(\pi) \prod_{\kappa^{\prime}=1}^{\kappa} f_{\kappa^{\prime} \pi\left(\kappa^{\prime}\right)} \sum_{\chi \in \operatorname{Perm}(\lambda)} \operatorname{sgn}(\chi) \prod_{\lambda^{\prime}=1}^{\lambda} f_{\lambda^{\prime} \chi\left(\lambda^{\prime}\right)} \\
& \operatorname{det}\left(R\left[J_{0}, K_{0} ; J_{0}, K_{0}\right]\right) \\
= & \operatorname{det}\left(f_{\mu \nu}\right)_{\mu, \nu=1}^{\kappa} \operatorname{det}\left(f_{\mu \nu}\right)_{\mu, \nu=1}^{\lambda} \operatorname{det}\left(R\left[J_{0}, K_{0} ; J_{0}, K_{0}\right]\right) .
\end{aligned}
$$

For the evaluation of the first two determinants see Lemma 9.5. The evaluation of the last one is in fact quite involved, and we refer to [24, Theorem 5.1] for the fact ${ }^{2}$ that $\operatorname{det}\left(R\left[J_{0}, K_{0} ; J_{0}, K_{0}\right]\right)=(-1)^{\kappa+\lambda} C^{2} C_{\kappa \lambda}$ for $\lambda=\kappa-1, \kappa$. Hence,

$$
D_{\kappa \lambda}=(-1)^{\frac{\kappa(\kappa+1)}{2}}(-1)^{\frac{\lambda(\lambda+1)}{2}} \frac{\prod_{\kappa^{\prime}=1}^{\kappa-1} \kappa^{\prime} !}{\prod_{\kappa^{\prime}=1}^{\kappa}\left(n_{k}-\kappa^{\prime}\right) !} \frac{\prod_{\lambda^{\prime}=1}^{\lambda-1} \lambda^{\prime} !}{\prod_{\lambda^{\prime}=1}^{\lambda}\left(n_{k}-\lambda^{\prime}\right) !} C^{2} C_{\kappa \lambda} .
$$

Using (9.7), 9.8) in 9.5, we infer

$$
\begin{aligned}
\operatorname{det}(S[J, K ; J, K])=(-1)^{\frac{\kappa(\kappa+1)}{2}}(-1)^{\frac{\lambda(\lambda+1)}{2}} C^{2} C_{\kappa \lambda} F_{\kappa}(t) \overline{F_{\lambda}(t)} \\
|t|^{(\kappa+\lambda) \rho} t^{\kappa\left(n_{k}-\kappa\right)+\lambda\left(n_{k}-\lambda\right)} \quad\left[1+\mathcal{O}\left(\frac{\log |t|}{|t|}\right)\right]
\end{aligned}
$$

and the proof is completed by inserting 9.9 into 9.2 .

Now we are in position to give the asymptotic behavior of the $k$ th wave packet.

${ }^{2}$ For the reader's convenience we sketch how to apply [24 Theorem 5.1] in the situation at hand. First we state

Corollary 9.6. Let $l_{i}, m_{j} \in \mathbb{N}, i=1, \ldots, L, j=1, \ldots, M$, and $l=\sum_{i=1}^{L} l_{i}, m=\sum_{j=1}^{M} m_{j}$.

For $\beta_{i}, \gamma_{j} \in \mathbb{C}, i=1, \ldots, L, j=1, \ldots, M$, with $\beta_{i}+\gamma_{j} \neq 0$ for all $i, j$, we define the matrix $U=\left(U_{i j}\right)_{\substack{i=1, \ldots, L \\ j=1, \ldots, M}} \in \mathcal{M}_{l, m}(\mathbb{C})$ with the blocks

$$
U_{i j}=\left(\left(\frac{1}{\beta_{i}+\gamma_{j}}\right)^{i^{\prime}+j^{\prime}-1}\left(\begin{array}{c}
i^{\prime}+j^{\prime}-2 \\
i^{\prime}-1
\end{array}\right)\right)_{\substack{i^{\prime}=1, \ldots, l_{i} \\
j^{\prime}=1, \ldots, m_{j}}} \in \mathcal{M}_{l_{i}, m_{j}}(\mathbb{C}) .
$$

Furthermore, let $g$ be the vector consisting of the first standard basis vectors $e_{m_{j}}^{(1)} \in \mathbb{C}^{m_{j}}$ for $j=1, \ldots, M$. Then, for $m \in\{l, l+1\}$,

$$
\operatorname{det}\left(\begin{array}{cc}
-g g^{t r} & -U \\
U^{t r} & 0
\end{array}\right)=(-1)^{l+m} \prod_{\substack{i, j=1 \\
i<j}}^{L}\left(\beta_{i}-\beta_{j}\right)^{2 l_{i} l_{j}} \prod_{\substack{i, j=1 \\
i<j}}^{M}\left(\gamma_{i}-\gamma_{j}\right)^{2 m_{i} m_{j}} / \prod_{i=1}^{L} \prod_{j=1}^{M}\left(\beta_{i}+\gamma_{j}\right)^{2 l_{i} m_{j}} .
$$

Corollary 9.6 immediately follows from [24, Theorem 5.1] using the fact that $U$ and $U^{t r}$ have the same structure, and the following identity

$$
\left(\begin{array}{cc}
-g g^{t r} & -U \\
U^{t r} & 0
\end{array}\right)=-\left(\begin{array}{cc}
0_{n \times m} & I_{n} \\
I_{m} & 0_{m \times n}
\end{array}\right)\left(\begin{array}{cc}
0 & -U^{t r} \\
U & g g^{t r}
\end{array}\right)\left(\begin{array}{cc}
0_{n \times m} & I_{n} \\
I_{m} & 0_{m \times n}
\end{array}\right)
$$

which holds for all $g \in \mathbb{C}^{m}$ and $U \in \mathcal{M}_{m, n}(\mathbb{C})$.

To conclude, we apply Corollary 9.6 with $L=M=k, \beta_{j}=\alpha_{j}, \gamma_{j}=\bar{\alpha}_{j}, l_{j}=m_{j}=n_{j}$ for all $j<k$ and $l_{k}=\kappa, m_{k}=\lambda$. 
Proposition 9.7. The $k$ th wave packet $q^{(k)}$ given in Proposition 8.1 satisfies:

$$
q^{(k)}(x, t) \approx \sum_{k^{\prime}=0}^{n_{k}-1} q^{\left(k k^{\prime}\right)}(x, t) \text { for } t \approx-\infty,
$$

where

$$
q^{\left(k k^{\prime}\right)}(x, t)=\epsilon^{n_{k}-1} \operatorname{Re}\left(\alpha_{k}\right) \frac{2 e^{\Delta_{k k^{\prime}}^{-}(x, t)}}{1+e^{2 \operatorname{Re}\left(\Delta_{k k^{\prime}}^{-}(x, t)\right)}} .
$$

Here $\epsilon=\operatorname{sgn}(t), K^{\prime}=-\left(n_{k}-1\right)+2 k^{\prime}$ is the index associated to $k^{\prime}$, and the function $\Delta_{k k^{\prime}}^{-}$is

$$
\Delta_{k k^{\prime}}^{-}(x, t)=\alpha_{k} x-\mathrm{i} \alpha_{k}^{2} t-K^{\prime} \log |t|+\varphi_{k}+\varphi_{k}^{-}-\varphi_{k k^{\prime}}^{-}
$$

with $\varphi_{k}, \varphi_{k}^{-}, \varphi_{k k^{\prime}}^{-}$as defined in Theorem 3.3 .

Note that in the above proposition the term $\epsilon=\operatorname{sgn}(t)$ is kept deliberately in order to simplify the transition to the case $t \rightarrow+\infty$.

Proof. Recall $\operatorname{Re}\left(\alpha_{k}\right)>0$. We may assume $t<-1$. (This is only needed to guarantee that the intervals defined below have positive length).

Fix $k^{\prime}$ and let $K^{\prime}=-\left(n_{k}-1\right)+2 k^{\prime}$. Consider

$$
\mathcal{I}_{k^{\prime}}(t)=\left(\gamma_{K^{\prime}-\frac{1}{2}}(t), \gamma_{K^{\prime}+\frac{1}{2}}(t)\right),
$$

where $\gamma_{\rho}(t)=v_{k} t+\rho \log |t| / \operatorname{Re}\left(\alpha_{k}\right)$. This interval has the center $\gamma_{K^{\prime}}(t)$, and its diameter grows logarithmically with $|t|$. Moreover, define

$$
\mathcal{C}_{k^{\prime}}=\bigcup_{t \leq-1} \mathcal{I}_{k^{\prime}}(t)
$$

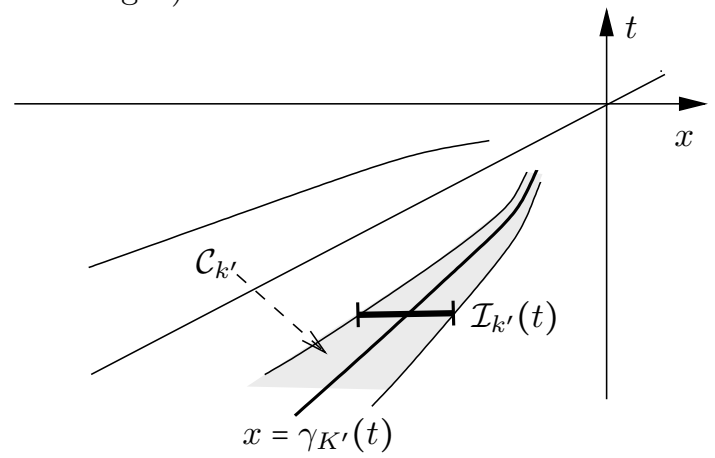

The proof is organized in three steps in which we show that the following properties hold asymptotically:

Step 1: $q^{(k)} \approx q^{\left(k k^{\prime}\right)}$ in $\mathcal{C}_{k^{\prime}}$, i.e. the only contribution comes from the $k^{\prime}$ th soliton $q^{\left(k k^{\prime}\right)}$,

Step 2: $q^{\left(k k^{\prime}\right)} \approx 0$ outside $\mathcal{C}_{k^{\prime}}$,

Step 3: $q^{(k)} \approx 0$ outside $\bigcup_{k^{\prime}=0}^{n_{k}-1} \mathcal{C}_{k^{\prime}}$.

Step 1. Let $x \in \mathcal{I}_{k^{\prime}}(t)$. Then $x=\gamma_{K^{\prime}+\rho}(t)$ with $\rho \in\left(-\frac{1}{2}, \frac{1}{2}\right)$.

To start with we identify in $P^{(k)}\left(\gamma_{K^{\prime}+\rho}(t), t\right), p^{(k)}\left(\gamma_{K^{\prime}+\rho}(t), t\right)$ the terms of leading order in $t$. According to Proposition 9.1, the relevant exponents are:

$$
f_{\rho}(\kappa) \text { for } \kappa \in\left\{0, \ldots, n_{k}\right\}, \quad g_{\rho}(\kappa) \text { for } \kappa \in\left\{0, \ldots, n_{k}-1\right\},
$$

where

$$
\begin{aligned}
& f_{\rho}(\kappa)=-2 \kappa^{2}+2\left(n_{k}+K^{\prime}+\rho\right) \kappa, \\
& g_{\rho}(\kappa)=-2 \kappa^{2}+2\left(n_{k}-1+K^{\prime}+\rho\right) \kappa+\left(n_{k}-1+K^{\prime}+\rho\right) .
\end{aligned}
$$

The graph of $f_{\rho}$ (regarding $\kappa$ as a continuous variable) is a concave parabola, and $f_{\rho}$ attains its global maximum at $\kappa_{0}=\left(n_{k}+K^{\prime}+\rho\right) / 2=\left(k^{\prime}+\frac{1}{2}\right)+\frac{\rho}{2}$. Since $|\rho|<\frac{1}{2}$, candidates for the leading exponents correspond to the indices $\kappa_{1}=k^{\prime}$ and $\kappa_{2}=k^{\prime}+1$. 
Furthermore, we use $f_{\rho}\left(k^{\prime}+1\right)=f_{\rho}\left(k^{\prime}\right)+2 \rho, f_{\rho}\left(k^{\prime}+2\right)=f_{\rho}\left(k^{\prime}-1\right)+6 \rho$ to estimate the minimal distance to the other exponents from below by

$$
\begin{aligned}
& \min \left(f_{\rho}\left(k^{\prime}\right), f_{\rho}\left(k^{\prime}+1\right)\right)-\max \left(f_{\rho}\left(k^{\prime}-1\right), f_{\rho}\left(k^{\prime}+2\right)\right)= \\
& =\left\{\begin{array}{cl}
f_{\rho}\left(k^{\prime}+1\right)-f_{\rho}\left(k^{\prime}-1\right) & \text { for } \rho \leq 0 \\
f_{\rho}\left(k^{\prime}\right)-f_{\rho}\left(k^{\prime}+2\right) & \text { for } \rho \geq 0
\end{array}\right. \\
& =4(1-|\rho|)>2 .
\end{aligned}
$$

Analogously, $g_{\rho}$ has its maximum at $\kappa_{0}=\left(n_{k}-1+K^{\prime}+\rho\right) / 2=k^{\prime}+\frac{\rho}{2}$, showing that the leading exponent corresponds to $\kappa_{1}=k^{\prime}$, and for the minimal distance to the other exponents we get the estimate $g_{\rho}\left(k^{\prime}\right)-\max \left(g_{\rho}\left(k^{\prime}-1\right), g_{\rho}\left(k^{\prime}+1\right)\right)>1$.

Keeping only the terms of leading order in $t$ (which correspond to $\kappa=k^{\prime}, k^{\prime}+1$ for $p^{(k)}$ and $\kappa=k^{\prime}$ for $p^{(k)}-P^{(k)}$ in Proposition 9.1), we get for $q^{(k)}=1-P^{(k)} / p^{(k)}=$ $\left(p^{(k)}-P^{(k)}\right) / p^{(k)}$,

$$
\begin{aligned}
& q^{(k)}\left(\gamma_{K^{\prime}+\rho}(t), t\right)= \\
& \quad=\frac{-(-1)^{k^{\prime}+1} C_{\left(k^{\prime}+1\right) k^{\prime}} G_{k^{\prime}}(t)|t|^{K^{\prime}+\rho} t^{n_{k}-2 k^{\prime}-1}\left[1+\mathcal{O}\left(\frac{\log |t|}{|t|}\right)\right]}{\left(C_{k^{\prime} k^{\prime}}+C_{\left(k^{\prime}+1\right)\left(k^{\prime}+1\right)} G_{k^{\prime}}(t) \overline{G_{k^{\prime}}(t)}|t|^{2\left(K^{\prime}+\rho\right)} t^{2\left(n_{k}-2 k^{\prime}-1\right)}\right)\left[1+\mathcal{O}\left(\frac{\log |t|}{|t|}\right)\right]} \\
& \quad=\epsilon^{K^{\prime}}(-1)^{k^{\prime}} \frac{C_{\left(k^{\prime}+1\right) k^{\prime}} G_{k^{\prime}}(t)|t|^{\rho}}{C_{k^{\prime} k^{\prime}}+C_{\left(k^{\prime}+1\right)\left(k^{\prime}+1\right)} G_{k^{\prime}}(t) \overline{G_{k^{\prime}}(t)}|t|^{2 \rho}}\left[1+\mathcal{O}\left(\frac{\log |t|}{|t|}\right)\right]
\end{aligned}
$$

with

$$
G_{k^{\prime}}(t)=\frac{F_{k^{\prime}+1}(t)}{F_{k^{\prime}}(t)}=\frac{k^{\prime} !}{\left(n_{k}-k^{\prime}-1\right) !} d_{k}^{n_{k}-2 k^{\prime}-1} b_{k}^{\left(n_{k}\right)} e^{\mathrm{i} \Upsilon^{(k)}\left(\gamma_{K^{\prime}+\rho}(t), t\right)} .
$$

Recall $b_{k}^{\left(n_{k}\right)}=a_{k}^{(1)} c_{k}^{\left(n_{k}\right)}$, see Proposition 7.1, $d_{k}=-2 \mathrm{iRe}\left(\alpha_{k}\right)$, see Proposition 9.1, and $K^{\prime}=-\left(n_{k}-1\right)+2 k^{\prime}$. Using also the definitions of $\varphi_{k}, \varphi_{k k^{\prime}}^{-}$, and $\varphi_{k}^{-}$in Theorem 3.3 , we find

$$
\begin{aligned}
& G_{k^{\prime}}(t) e^{-\mathrm{i} \Upsilon^{(k)}\left(\gamma_{K^{\prime}+\rho}(t), t\right)}=\frac{k^{\prime} !}{\left(k^{\prime}-K^{\prime}\right) !}(-\mathrm{i})^{-K^{\prime}}\left(2 \operatorname{Re}\left(\alpha_{k}\right)\right)^{-K^{\prime}} a_{k}^{(1)} c_{k}^{\left(n_{k}\right)} \\
& =(-1)^{k^{\prime}}\left(2 \operatorname{Re}\left(\alpha_{k}\right)\right)^{2 k^{\prime}+1} \frac{k^{\prime} !}{\left(k^{\prime}-K^{\prime}\right) !}\left(2 \operatorname{Re}\left(\alpha_{k}\right)\right)^{-2 K^{\prime}}(-\mathrm{i})^{n_{k}-1} \frac{a_{k}^{(1)} c_{k}^{\left(n_{k}\right)}}{\left(2 \operatorname{Re}\left(\alpha_{k}\right)\right)^{n_{k}}} \\
& =\left(2 \operatorname{Re}\left(\alpha_{k}\right)\right)^{2 k^{\prime}+1} e^{\varphi_{k}-\varphi_{k k^{\prime}}^{-}} \\
& \frac{C_{\left(k^{\prime}+1\right) k^{\prime}}}{C_{k^{\prime} k^{\prime}}}=\frac{e^{\varphi_{k}^{-}}}{\left(2 \operatorname{Re}\left(\alpha_{k}\right)\right)^{2 k^{\prime}}}, \quad \frac{C_{\left(k^{\prime}+1\right)\left(k^{\prime}+1\right)}}{C_{k^{\prime} k^{\prime}}}=\frac{e^{\varphi_{k}^{-}+\varphi_{k}^{-}}}{\left(2 \operatorname{Re}\left(\alpha_{k}\right)\right)^{4 k^{\prime}+2}}
\end{aligned}
$$

Inserting this into 9.12 , we get $q^{(k)}\left(\gamma_{K^{\prime}+\rho}(t), t\right)=\mathcal{Q}^{\left(k k^{\prime}\right)}(t)\left[1+\mathcal{O}\left(\frac{\log |t|}{|t|}\right)\right]$, where

$$
\begin{aligned}
& \mathcal{Q}^{\left(k k^{\prime}\right)}(t)=(-1)^{k^{\prime}} \epsilon^{K^{\prime}} 2 \operatorname{Re}\left(\alpha_{k}\right) \frac{\mathcal{P}^{\left(k k^{\prime}\right)}(t)|t|^{\rho}}{1+\mathcal{P}^{\left(k k^{\prime}\right)}(t) \overline{\mathcal{P}^{\left(k k^{\prime}\right)}(t)}|t|^{2 \rho}}, \\
& \mathcal{P}^{\left(k k^{\prime}\right)}(t)=\exp \left(\operatorname{i\Upsilon }^{(k)}\left(\gamma_{K^{\prime}+\rho}(t), t\right)+\varphi_{k}+\varphi_{k}^{-}-\varphi_{k k^{\prime}}^{-}\right) .
\end{aligned}
$$

Since $\mathcal{Q}^{\left(k k^{\prime}\right)}(t)$ is bounded, it even holds $q^{(k)}\left(\gamma_{K^{\prime}+\rho}(t), t\right)=\mathcal{Q}^{\left(k k^{\prime}\right)}(t)+\mathcal{O}\left(\frac{\log |t|}{|t|}\right)$. 
On the other hand it is straightforward to check that $q^{\left(k k^{\prime}\right)}\left(\gamma_{K^{\prime}+\rho}(t), t\right)=\mathcal{Q}^{\left(k k^{\prime}\right)}(t)$. Hence we may conclude

$$
\sup _{x \in \mathcal{I}_{k^{\prime}}(t)}\left|q^{(k)}(x, t)-q^{\left(k k^{\prime}\right)}(x, t)\right| \rightarrow 0 \text { as } t \rightarrow-\infty .
$$

In summary,

$$
\mathbb{1}_{\mathcal{C}_{k^{\prime}}} q^{(k)} \approx \mathbb{1}_{\mathcal{C}_{k^{\prime}}} q^{\left(k k^{\prime}\right)} \text { for } t \approx-\infty
$$

where $\mathbb{1}_{\mathcal{C}_{k^{\prime}}}$ is the characteristic function of $\mathcal{C}_{k^{\prime}}$, and Step $\mathbf{1}$ is complete.

Step 2. To discuss the behavior of $q^{\left(k k^{\prime}\right)}$ outside of $\mathcal{I}_{k^{\prime}}(t)$, we distinguish two cases:

First, let $x \in \mathcal{I}_{k^{\prime}}^{-}(t)=\left(-\infty, \gamma_{K^{\prime}-\frac{1}{2}}(t)\right]$. Then $x=\gamma_{K^{\prime}+\rho}(t)$ with $\rho \leq-\frac{1}{2}$, and

$$
\left|q^{\left(k k^{\prime}\right)}\left(\gamma_{K^{\prime}+\rho}(t), t\right)\right|=\mid \mathcal{Q}^{\left(k k^{\prime}\right)}(t) \stackrel{9.14}{-} 2 \operatorname{Re}\left(\alpha_{k}\right) \frac{c|t|^{\rho}}{1+c^{2}|t|^{2 \rho}}=\mathcal{O}\left(|t|^{\rho}\right),
$$

where we have used 9.15 to see that $c=\left|\mathcal{P}^{\left(k k^{\prime}\right)}(t)\right|>0$ does not depend on $t$. Hence $\left|q^{\left(k k^{\prime}\right)}\left(\gamma_{K^{\prime}+\rho}(t), t\right)\right|=\mathcal{O}\left(|t|^{-\frac{1}{2}}\right)$ for all $\rho \leq-\frac{1}{2}$.

Second, let $x \in \mathcal{I}_{k^{\prime}}^{+}(t)=\left[\gamma_{K^{\prime}+\frac{1}{2}}(t), \infty\right)$. Then $x=\gamma_{K^{\prime}+\rho}(t)$ with $\rho \geq \frac{1}{2}$, and after rewriting

$$
\frac{c|t|^{\rho}}{1+c^{2}|t|^{2 \rho}}=\frac{|t|^{-\rho} / c}{1+|t|^{-2 \rho} / c^{2}},
$$

a similar argument as above shows $\left|q^{\left(k k^{\prime}\right)}\left(\gamma_{K^{\prime}+\rho}(t), t\right)\right|=\mathcal{O}\left(|t|^{-\frac{1}{2}}\right)$ for all $\rho \geq \frac{1}{2}$.

This completes Step 2 as we have shown

$$
\left(1-\mathbb{1}_{\mathcal{C}_{k^{\prime}}}\right) q^{\left(k k^{\prime}\right)} \approx 0 \text { for } t \approx-\infty
$$

Step 3. To discuss the behavior of $q^{(k)}$ outside $\cup_{k^{\prime}=0}^{n_{k}-1} \mathcal{C}_{k^{\prime}}$, we distinguish three cases. Consider first the interval

$$
\mathcal{J}_{k^{\prime}}(t)=\left[\gamma_{K^{\prime}+\frac{1}{2}}(t), \gamma_{\left(K^{\prime}+2\right)-\frac{1}{2}}(t)\right]
$$

for $k^{\prime}<n_{k}-1$. Note that, if $K^{\prime}$ is the index associated to $k^{\prime}$, then $K^{\prime}+2$ is the index associated to $k^{\prime}+1$. Thus, $\mathcal{J}_{k^{\prime}}(t)$ is the interval covering the gap between $\mathcal{I}_{k^{\prime}}(t)$ and $\mathcal{I}_{k^{\prime}+1}(t)$. Let $x \in \mathcal{J}_{k^{\prime}}(t)$, then $x=\gamma_{K^{\prime}+\rho}(t)$ with $\rho \in\left[\frac{1}{2}, \frac{3}{2}\right]$.

To find the terms of leading order in $t$ for $P^{(k)}\left(\gamma_{K^{\prime}+\rho}(t), t\right), p^{(k)}\left(\gamma_{K^{\prime}+\rho}(t), t\right)$, recall that the function $f_{\rho}$ given in 9.10 has its maximum at $\kappa_{0}=\left(k^{\prime}+\frac{1}{2}\right)+\frac{\rho}{2}$. Since $\frac{1}{2} \leq \rho \leq \frac{3}{2}$, the leading exponent corresponds to $\kappa_{1}=k^{\prime}+1$, and its minimal distance to the other exponents can be estimated from below by

$$
f_{\rho}\left(k^{\prime}+1\right)-\max \left(f_{\rho}\left(k^{\prime}\right), f_{\rho}\left(k^{\prime}+2\right)\right)=\min (2 \rho, 4-2 \rho) \geq 1 .
$$

Since the function $g_{\rho}$ given in 9.11 has its maximum at $\kappa_{0}=k^{\prime}+\frac{\rho}{2}$, candidates for the leading exponent are $\kappa_{1}=k^{\prime}$ and $\kappa_{2}=k^{\prime}+1$, and for the minimal distance to the other exponents we get

$$
\begin{aligned}
& \min \left\{g_{\rho}\left(k^{\prime}\right), g_{\rho}\left(k^{\prime}+1\right)\right\}-\max \left\{g_{\rho}\left(k^{\prime}-1\right), g_{\rho}\left(k^{\prime}+2\right)\right\}= \\
& =\left\{\begin{array}{c}
g_{\rho}\left(k^{\prime}+1\right)-g_{\rho}\left(k^{\prime}-1\right)=4 \rho \geq 2 \\
g_{\rho}\left(k^{\prime}\right)-g_{\rho}\left(k^{\prime}+2\right)=4(2-\rho) \geq 2
\end{array} \quad \text { for } \rho \leq 1,\right.
\end{aligned}
$$

Keeping only the terms of leading order in $t$, we get

$q^{(k)}\left(\gamma_{K^{\prime}+\rho}(t), t\right)=$ 


$$
\begin{aligned}
& =\frac{-\left((-1)^{k^{\prime}+1} C_{\left(k^{\prime}+1\right) k^{\prime}}+(-1)^{k^{\prime}+2} C_{\left(k^{\prime}+2\right)\left(k^{\prime}+1\right)} G_{k^{\prime}+1}(t) \overline{G_{k^{\prime}}(t)}|t|^{2\left(K^{\prime}+\rho\right)} t^{2\left(n_{k}-2 k^{\prime}-2\right)}\right)}{C_{\left(k^{\prime}+1\right)\left(k^{\prime}+1\right)} \overline{G_{k^{\prime}}(t)}|t|^{K^{\prime}+\rho} t^{n_{k}-2 k^{\prime}-1}} \\
& =(-1)^{k^{\prime}} \epsilon^{-K^{\prime}} \frac{C_{\left(k^{\prime}+1\right) k^{\prime}}-C_{\left(k^{\prime}+2\right)\left(k^{\prime}+1\right)} G_{k^{\prime}+1}(t) \overline{G_{k^{\prime}}(t)}|t|^{2 \rho-2}}{C_{\left(k^{\prime}+1\right)\left(k^{\prime}+1\right)} \overline{G_{k^{\prime}}(t)}|t|^{\rho}}\left[1+\mathcal{O}\left(\frac{\log |t|}{|t|}\right)\right]
\end{aligned}
$$

with $G_{k^{\prime}}(t)$ as in 9.13$)$. Observe that $\left|G_{k^{\prime}}(t)\right|=c_{k^{\prime}}$ does not depend on $t$. Hence, for all $\frac{1}{2} \leq \rho \leq \frac{3}{2}$,

$$
\begin{aligned}
\mid q^{(k)} & \left(\gamma_{K^{\prime}+\rho}(t), t\right) \mid \\
& \leq\left(\frac{1}{c_{k^{\prime}}} \frac{C_{\left(k^{\prime}+1\right) k^{\prime}}}{C_{\left(k^{\prime}+1\right)\left(k^{\prime}+1\right)}}|t|^{-\rho}+c_{k^{\prime}+1} \frac{C_{\left(k^{\prime}+2\right)\left(k^{\prime}+1\right)}}{C_{\left(k^{\prime}+1\right)\left(k^{\prime}+1\right)}}|t|^{\rho-2}\right)\left[1+\mathcal{O}\left(\frac{\log |t|}{|t|}\right)\right] \\
& =\mathcal{O}\left(|t|^{-\frac{1}{2}}\right) .
\end{aligned}
$$

Second, consider the interval $\mathcal{J}_{\min }(t)=\left(-\infty, \gamma_{\left(-\left(n_{k}-1\right)-\frac{1}{2}\right)}(t)\right]$. Note that $\mathcal{J}_{\min }(t)$ covers the gap between $-\infty$ and $\mathcal{I}_{0}(t)$. Let $x \in \mathcal{J}_{\min }(t)$, then $x=\gamma_{\left(-\left(n_{k}-1\right)+\rho\right)}(t)$ with $\rho \in\left(-\infty,-\frac{1}{2}\right]$.

In this case the leading exponent both for $f_{\rho}$ and $g_{\rho}$ corresponds to $\kappa_{1}=0$, and for the minimal distance to the other exponents we get $f_{\rho}(0)-f_{\rho}(1)=-2 \rho \geq 1$ and $g_{\rho}(0)-g_{\rho}(1)=-2(\rho-1) \geq 3$. Hence,

$$
q^{(k)}\left(\gamma_{\left(-\left(n_{k}-1\right)+\rho\right)}(t), t\right)=\epsilon^{n_{k}-1} \frac{C_{10}}{C_{00}} G_{0}(t)|t|^{\rho}\left[1+\mathcal{O}\left(\frac{\log |t|}{|t|}\right)\right],
$$

showing $\left|q^{(k)}\left(\gamma_{\left(-\left(n_{k}-1\right)+\rho\right)}(t), t\right)\right|=\mathcal{O}\left(t^{-\frac{1}{2}}\right)$ for all $\rho \leq-\frac{1}{2}$.

Finally consider $\mathcal{J}_{\text {max }}(t)=\left[\gamma_{\left(\left(n_{k}-1\right)+\frac{1}{2}\right)}(t), \infty\right)$, the interval covering the gap between $\mathcal{I}_{n_{k}-1}(t)$ and $+\infty$. Let $x \in \mathcal{J}_{\max }(t)$, then $x=\gamma_{\left(\left(n_{k}-1\right)+\rho\right)}(t)$ with $\rho \in\left[\frac{1}{2}, \infty\right)$.

Here the leading exponents correspond to $\kappa_{1}=n_{k}$ for $f_{\rho}$ and $\kappa_{2}=n_{k}-1$ for $g_{\rho}$, and for the minimal distance to the other exponents we have $f_{\rho}\left(n_{k}\right)-f_{\rho}\left(n_{k}-1\right)=2 \rho \geq 1$ and $g_{\rho}\left(n_{k}-1\right)-g_{\rho}\left(n_{k}-2\right)=2(\rho+1) \geq 3$. Hence

$$
\left.q^{(k)} \gamma_{\left(\left(n_{k}-1\right)+\rho\right)}(t), t\right)=(-\epsilon)^{n_{k}-1} \frac{C_{n_{k}\left(n_{k}-1\right)}}{C_{n_{k} n_{k}}} \frac{1}{G_{n_{k}-1}(t)}|t|^{-\rho}\left[1+\mathcal{O}\left(\frac{\log |t|}{|t|}\right)\right],
$$

showing $\left|q^{(k)}\left(\gamma_{\left(\left(n_{k}-1\right)+\rho\right)}(t), t\right)\right|=\mathcal{O}\left(|t|^{-\frac{1}{2}}\right)$ for all $\rho \geq \frac{1}{2}$.

As a result,

$$
\left(1-\sum_{k^{\prime}=0}^{n_{k}-1} \mathbb{1}_{\mathcal{C}_{k^{\prime}}}\right) q^{(k)} \approx 0 \text { for } t \approx-\infty .
$$

This completes Step 3 and, by a similar conclusion as in the proof of Proposition 8.1. also the proof of Proposition 9.7.

Proof of Theorem 3.3. Theorem 3.3 follows from Propositions 8.1 and 9.7 upon reordering as follows: Set $k^{\prime \prime}=\left(n_{k}-1\right)-k^{\prime}$. For the index $K^{\prime \prime}=-\left(n_{k}-1\right)+2 k^{\prime \prime}$ associated to $k^{\prime \prime}$, we then have

$$
K^{\prime \prime}=-K^{\prime} \text { and } k^{\prime \prime}-K^{\prime \prime}=k^{\prime} .
$$

In particular, $\varphi_{k k^{\prime \prime}}^{-}=-\varphi_{k k^{\prime}}^{-}$. Geometrically, this replacement reverses the order of the solitons within the $k$ th wave packet. 
Remark 9.8. To see that the asymptotic description in Theorem 3.3 includes what is proved in 30. (for $N$-solitons) and [15] (for a single wave packet), one proceeds as follows:

(1) In Section 6, it is shown that the solutions obtained via ISM starting from the data 6.1) are realized in Theorem 2.3 for

$$
\widehat{A}=-2 \mathrm{i} J, \quad \widehat{a}=-2 \mathrm{i}\left(e_{n_{j}}^{(1)}\right)_{j=1}^{N}, \quad \widehat{c}=\left(\left(\overline{r_{j j^{\prime}}}\right)_{j^{\prime}=1}^{n_{j}}\right)_{j=1}^{N},
$$

where $J$ is in Jordan form with $N$ Jordan blocks of sizes $n_{j} \times n_{j}$ corresponding to eigenvalues $k_{j}$.

(2) To apply Theorem $3.3, \widehat{A}$ needs to be brought in Jordan form. This can be done using the transformation matrix $U=\operatorname{diag}\left\{U_{j} \mid j=1, \ldots, N\right\}$ with $U_{j}=\operatorname{diag}\left\{(-2 \mathrm{i})^{j^{\prime}-1} \mid j^{\prime}=1, \ldots, n_{j}\right\}$. Then $A=U^{-1} \widehat{A} U$ is in Jordan form.

Now Lemma 5.2 tells that the solution is not changed if we simultaneously replace $\widehat{A}, \widehat{a}, \widehat{c}$ by $A, a=\left(U^{-1}\right)^{\operatorname{tr}} \widehat{a}, c=U \widehat{c}$, which gives us as relevant data

$$
\alpha_{j}=-2 \mathrm{i} \overline{k_{j}}, \quad a_{j}^{(1)}=-2 \mathrm{i}, \quad c_{j}^{\left(n_{j}\right)}=(-2 \mathrm{i})^{n_{j}-1} \overline{r_{j n_{j}}} .
$$

(3) Since $\alpha_{j}=-2 \mathrm{i} \overline{k_{j}}$, we have $\operatorname{Re}\left(\alpha_{j}\right)<0$. As a consequence, in Theorem 3.3 the index sets $\Lambda_{j}^{+}$and $\Lambda_{j}^{-}$have to be interchanged (see also the paragraph on Assumption a) in Theorem 3.3 in Section 5).

(4) For the asymptotic form in $t \approx-\infty$, the reordering $j^{\prime \prime}=\left(n_{j}-1\right)-j^{\prime}$ can be used to switch from $\Gamma_{j j^{\prime}}^{-}(x, t)$ in Theorem 3.3 to

$$
\Gamma_{j j^{\prime \prime}}^{-}(x, t)=\alpha_{j} x-\mathrm{i} \alpha_{j}^{2} t-J^{\prime \prime} \log |t|+\varphi_{j}+\varphi_{j}^{-}+\varphi_{j j^{\prime \prime}}^{-}
$$

(compare the proof of Theorem 3.3.

(5) Note finally that the transformation $t \mapsto-t$ (in particular $\operatorname{sgn}(t) \mapsto \operatorname{sgn}(-t)$ ) is needed to bring the NLS in [15], 30] to the form used in this work.

\section{Solutions OF HigheR DEGENERACY}

This section aims at a first understanding of solutions involving solitons, or more generally wave packets of the same velocity. Motivated by [5], we start with the heuristic question whether groups of such wave packets should be considered as stable bound states. Then we indicate how our method contributes to the understanding of solutions of higher degeneracy by performing the asymptotic analysis for the first relevant case. We conclude with some plots for higher degeneracy phenomena in Figures 8- 10.

A family of degenerate solutions. Since wave packets which travel with the same velocity cannot be separated by asymptotic analysis, it is natural to ask whether they constitute stable bound states, so-called formations (see [3, p.34]). A famous example of such formations are the breather solutions of the sine-Gordon equation. In [19] it has been shown that these formations can be included in the asymptotic analysis.

For the NLS, a solution obtained by superposing two stationary solitons is discussed in [5, Example 7.2], see also Example 5.1. However, the subsequent example shows that this solution is not stable under collision with a non-stationary soliton. 

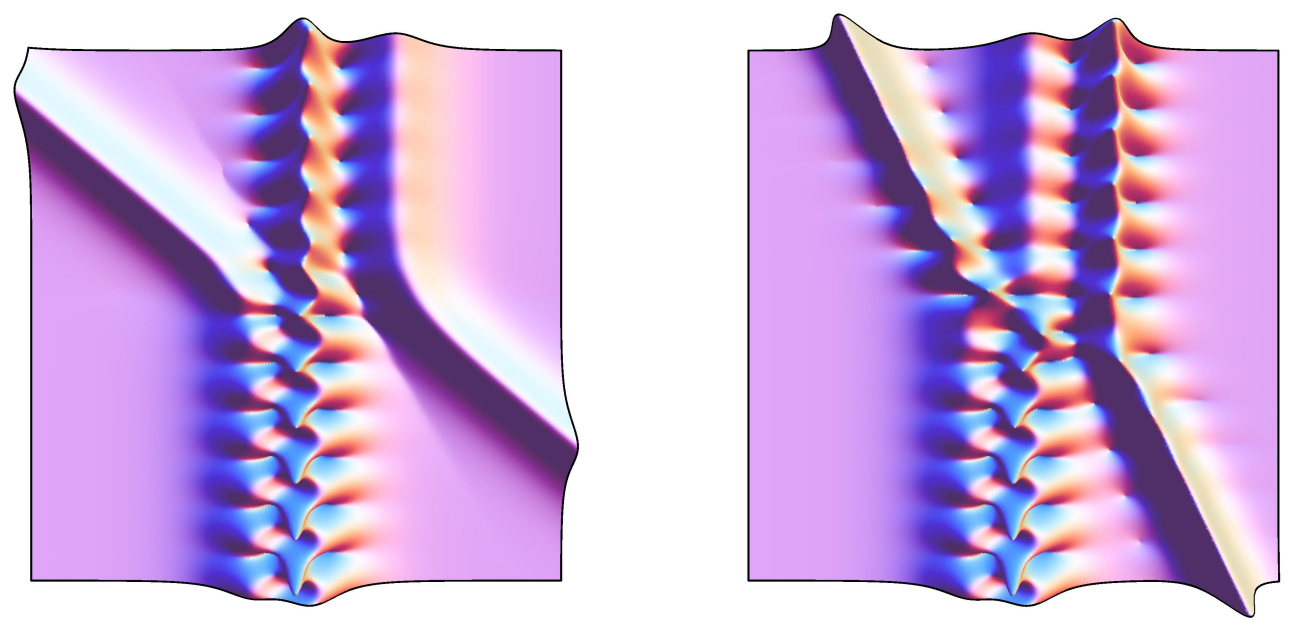

Figure 7. The solution discussed in Example 5.1 collides with non-stationary solitons, see Example 10.1 for details. In both cases, the two stationary solitons of which the solution in Example 5.1 consists are separated by the collision, thereby revealing its nature as a true 2-soliton. Note how sensitively the position-shift of the two stationary solitons depends on the non-stationary soliton: In the first picture the larger stationary soliton ends up on the left, in the second on the right side.

Example 10.1. Let $\operatorname{Im}(\beta) \neq 0$. Starting from the data

$$
A=\left(\begin{array}{ccc}
4 & 0 & 0 \\
0 & 2 & 0 \\
0 & 0 & \beta
\end{array}\right),
$$

we obtain a solution which superposes two stationary solitons of heights 4 and 2 respectively and a non-stationary soliton of height $|\operatorname{Re}(\beta)|$. Recall that the velocity of the non-stationary soliton is $-2 \operatorname{Im}(\beta)$. The corresponding solution is illustrated in Figure 10.1 for the parameters $a_{1}=a_{2}=1, c_{1}=18, c_{2}=-32$, and

- $\beta=2+\mathrm{i}$ with $a_{3}=1, c_{3}=4$ (for the plot to the left),

- $\beta=4+0.4 \mathrm{i}$ with $a_{3}=1, c_{3}=60$ (for the plot to the right).

In both cases the plotted region is $-5 \leq x \leq 5,-3 \leq t \leq 3$.

For large negative times this solution asymptotically looks like the superposition of the solution discussed in [5, Example 7.2], see also Example 5.1, and a moving soliton. But for large positive times, the two stationary solitons constituting the solution in [5, Example 7.2] appear clearly separated due to the fact that they have suffered different position-shifts in their collision with the moving soliton.

Asymptotic analysis of the degenerate 3-soliton. To confirm the heuristics of Example 10.1, we analyze in some detail the asymptotics of a 3-soliton with two solitons of the same velocity.

To this end, we consider the solution $q$ generated by $A=\operatorname{diag}\left\{\alpha_{1}, \alpha_{2}, \alpha_{3}\right\}$ with $\operatorname{Im}\left(\alpha_{1}\right)=\operatorname{Im}\left(\alpha_{2}\right) \neq \operatorname{Im}\left(\alpha_{3}\right)$ and $a=(1,1,1), c=\left(e^{\varphi_{1}}, e^{\varphi_{2}}, e^{\varphi_{3}}\right)$ in Theorem 2.3 . 
To avoid cancelation phenomena, let $\alpha_{1} \neq \alpha_{2}$. As before we will also assume $\operatorname{Re}\left(\alpha_{j}\right)>0$ for all $j$ (compare the paragraph on Assumption a) in Theorem 3.3 in Section 5p. We furthermore assume $\operatorname{Im}\left(\alpha_{3}\right)>\operatorname{Im}\left(\alpha_{1}\right)$, the case $\operatorname{Im}\left(\alpha_{3}\right)<\operatorname{Im}\left(\alpha_{1}\right)$ being analogous.

Observe that $q=1-P / p$, where $P, p$ are given by 2.5 with

$$
L=\left(\frac{1}{\alpha_{i}+\bar{\alpha}_{j}} \ell_{i}\right)_{i, j=1,2,3}, \quad L_{0}=\left(\ell_{i}\right)_{i, j=1,2,3},
$$

and $\ell_{i}(x, t)=\exp \left(\alpha_{i} x-\mathrm{i} \alpha_{i}^{2}+\varphi_{i}\right)$.

\section{Behavior for $t \rightarrow-\infty$.}

Step 1: Moving with the two solitons of equal velocity. Consider curves $x+2 \operatorname{Im}\left(\alpha_{1}\right) t=c$. On such a curve, $\left|\ell_{3}(x, t)\right| \rightarrow 0$ for $t \rightarrow-\infty$. The arguments for $P$, $p$ being parallel, we look at $P$ and find

$$
\begin{aligned}
& P \rightarrow \operatorname{det}\left(\begin{array}{cccccc}
-\ell_{1} & 1-\ell_{1} & -\ell_{1} & -\frac{1}{\alpha_{1}+\bar{\alpha}_{1}} \ell_{1} & -\frac{1}{\alpha_{1}+\bar{\alpha}_{2}} \ell_{1} & -\frac{1}{\alpha_{1}+\bar{\alpha}_{3}} \ell_{1} \\
-\ell_{2} & -\ell_{2} & 1-\ell_{2} & -\frac{1}{\alpha_{2}+\bar{\alpha}_{1}} \ell_{2} & -\frac{1}{\alpha_{2}+\bar{\alpha}_{2}} \ell_{2} & -\frac{1}{\alpha_{2}+\bar{\alpha}_{3}} \ell_{2} \\
0 & 0 & 1 & 0 & 0 & 0 \\
\frac{1}{\bar{\alpha}_{1}+\alpha_{1}} \bar{\ell}_{1} & \frac{1}{\bar{\alpha}_{1}+\alpha_{2}} \bar{\ell}_{1} & \frac{1}{\bar{\alpha}_{1}+\alpha_{3}} \bar{\ell}_{1} & 1 & 0 & 0 \\
\frac{1}{\bar{\alpha}_{2}+\alpha_{1}} \bar{\ell}_{2} & \frac{1}{\bar{\alpha}_{2}+\alpha_{2}} \bar{\ell}_{2} & \frac{1}{\bar{\alpha}_{2}+\alpha_{3}} \bar{\ell}_{2} & 0 & 1 & 0 \\
0 & 0 & 0 & 0 & 0 & 1
\end{array}\right) \\
& =\operatorname{det}\left(\begin{array}{cccc}
1-\ell_{1} & -\ell_{1} & -\frac{1}{\alpha_{1}+\bar{\alpha}_{1}} \ell_{1} & -\frac{1}{\alpha_{1}+\bar{\alpha}_{2}} \ell_{1} \\
-\ell_{2} & 1-\ell_{2} & -\frac{1}{\alpha_{2}+\bar{\alpha}_{1}} \ell_{2} & -\frac{\alpha_{1}+\bar{\alpha}_{2}}{\alpha_{2}} \ell_{2} \\
\frac{1}{\bar{\alpha}_{1}+\alpha_{1}} \bar{\ell}_{1} & \frac{1}{\bar{\alpha}_{1}+\alpha_{2}} \bar{\ell}_{1} & 1 & 0 \\
\frac{1}{\bar{\alpha}_{2}+\alpha_{1}} \bar{\ell}_{2} & \frac{1}{\bar{\alpha}_{2}+\alpha_{2}} \bar{\ell}_{2} & 0 & 1
\end{array}\right) .
\end{aligned}
$$

The corresponding solution is the superposition of the first two solitons as obtained from the $2 \times 2$-matrix $A=\operatorname{diag}\left\{\alpha_{1}, \alpha_{2}\right\}$ and $a=(1,1), c=\left(e^{\varphi_{1}}, e^{\varphi_{2}}\right)$ in Theorem 2.3. Note that, for $\alpha_{1}=4, \alpha_{2}=2$, and $c=(18,-32)$, this is is precisely the solution discussed in Example 5.1

Step 2: Moving With the Remaining SOliton. On curves $x+2 \operatorname{Im}\left(\alpha_{3}\right) t=c$, we observe $\left|1 / \ell_{1}(x, t)\right|,\left|1 / \ell_{2}(x, t)\right| \rightarrow 0$ for $t \rightarrow-\infty$. In this case we divide in both determinants $P, p$ the first row by $\ell_{1}$, the second row by $\ell_{2}$, the fourth row by $\bar{\ell}_{1}$, and the fifth row by $\bar{\ell}_{2}$. Note that this does not effect the quotient $P / p$. Again we focus on the more complicated determinant $P$, for which we get $P \rightarrow P_{0}$, where

$$
P_{0}=\operatorname{det}\left(\begin{array}{cccccc}
-1 & -1 & -1 & -\frac{1}{\alpha_{1}+\bar{\alpha}_{1}} & -\frac{1}{\alpha_{1}+\bar{\alpha}_{2}} & -\frac{1}{\alpha_{1}+\bar{\alpha}_{3}} \\
-1 & -1 & -1 & -\frac{1}{\alpha_{2}+\bar{\alpha}_{1}} & -\frac{1}{\alpha_{2}+\bar{\alpha}_{2}} & -\frac{1}{\alpha_{2}+\bar{\alpha}_{3}} \\
-\ell_{3} & -\ell_{3} & 1-\ell_{3} & -\frac{1}{\alpha_{3}+\bar{\alpha}_{1}} \ell_{3} & -\frac{1}{\alpha_{3}+\bar{\alpha}_{2}} \ell_{3} & -\frac{1}{\alpha_{3}+\bar{\alpha}_{3}} \ell_{3} \\
\frac{1}{\bar{\alpha}_{1}+\alpha_{1}} & \frac{1}{\bar{\alpha}_{1}+\alpha_{2}} & \frac{1}{\bar{\alpha}_{1}+\alpha_{3}} & 0 & 0 & 0 \\
\overline{\bar{\alpha}_{2}+\alpha_{1}} & \frac{1}{\bar{\alpha}_{2}+\alpha_{2}} & \frac{1}{\bar{\alpha}_{2}+\alpha_{3}} & 0 & 0 & 0 \\
\frac{1}{\bar{\alpha}_{3}+\alpha_{1}} \bar{\ell}_{3} & \frac{1}{\bar{\alpha}_{3}+\alpha_{2}} \bar{\ell}_{3} & \frac{1}{\bar{\alpha}_{3}+\alpha_{3}} \bar{\ell}_{3} & 0 & 0 & 1
\end{array}\right) .
$$

To identify the corresponding solution, we calculate $P_{0}$. Using the abbreviation $\gamma_{i j}=\left(\alpha_{i}-\alpha_{j}\right) /\left(\alpha_{i}+\bar{\alpha}_{j}\right)$, we perform the following operations simultaneously for both determinants:

(1) multiply the first row by $\left(\alpha_{1}+\bar{\alpha}_{1}\right) /\left(\alpha_{2}+\bar{\alpha}_{1}\right)$ and subtract it from the second row, extract the factor $\gamma_{21}$ from the second row, 
(2) multiply the first row by $\ell_{3}\left(\alpha_{1}+\bar{\alpha}_{1}\right) /\left(\alpha_{3}+\bar{\alpha}_{1}\right)$ and subtract it from the third row, set $f_{3}=\gamma_{31} \ell_{3}$,

(3) multiply the second row by $f_{3}\left(\alpha_{2}+\bar{\alpha}_{2}\right) /\left(\alpha_{3}+\bar{\alpha}_{2}\right)$ and subtract it from the third row, set $g_{3}=\gamma_{32} f_{3}$,

(4) multiply the first column by $\left(\bar{\alpha}_{1}+\alpha_{1}\right) /\left(\bar{\alpha}_{1}+\alpha_{2}\right)$ and subtract it from the second column, extract the factor $\gamma_{21}$ from the second column,

(5) multiply the first column by $\left(\bar{\alpha}_{1}+\alpha_{1}\right) /\left(\bar{\alpha}_{1}+\alpha_{3}\right)$ and subtract it from the third column,

(6) multiply the second column by $\gamma_{31}\left(\bar{\alpha}_{2}+\alpha_{2}\right) /\left(\bar{\alpha}_{2}+\alpha_{3}\right)$ and subtract it from the third column.

After expansion, this results in

$$
P_{0}=\gamma \operatorname{det}(I+T)
$$

with

$$
\gamma=\frac{\gamma_{21}^{2} \bar{\gamma}_{21}^{2}}{\left(\alpha_{1}+\bar{\alpha}_{1}\right)^{2}\left(\alpha_{2}+\bar{\alpha}_{2}\right)^{2}}, \quad T=\left(\begin{array}{cc}
-\gamma_{31} \gamma_{32} g_{3} & -\frac{\bar{\gamma}_{31} \bar{\gamma}_{32}}{\alpha_{3}+\bar{\alpha}_{3}} g_{3} \\
\frac{\gamma_{31} \gamma_{32}}{\bar{\alpha}_{3}+\alpha_{3}} \bar{g}_{3} & 0
\end{array}\right)
$$

Factorizing $T=T_{1} T_{2}$, where

$$
T_{1}=\left(\begin{array}{cc}
-g_{3} & -\frac{1}{\alpha_{3}+\bar{\alpha}_{3}} g_{3} \\
\frac{1}{\bar{\alpha}_{3}+\alpha_{3}} \bar{g}_{3} & 0
\end{array}\right), \quad T_{2}=\left(\begin{array}{cc}
\gamma_{31} \gamma_{32} & 0 \\
0 & \bar{\gamma}_{31} \bar{\gamma}_{32}
\end{array}\right),
$$

and using the identity $\operatorname{det}\left(I+T_{1} T_{2}\right)=\operatorname{det}\left(I+T_{2} T_{1}\right)$, we get

$$
P_{0}=\gamma \operatorname{det}\left(\begin{array}{cc}
1-h_{3} & -\frac{1}{\alpha_{3}+\bar{\alpha}_{3}} h_{3} \\
\frac{1}{\bar{\alpha}_{3}+\alpha_{3}} \bar{h}_{3} & 1
\end{array}\right)
$$

for $h_{3}=\left(\gamma_{31} \gamma_{32}\right)^{2} \ell_{3}$.

The factor $\gamma$ appears also in front of the other determinant and hence cancels out in the quotient. As a result, we find the third soliton as obtained from $A=\alpha_{3}$, $a=1, c=e^{\psi_{3}}$ in Theorem 2.3, with the initial shift

$$
\psi_{3}=\varphi_{3}+\log \left(\frac{\left(\alpha_{3}-\alpha_{1}\right)\left(\alpha_{3}-\alpha_{2}\right)}{\left(\alpha_{3}+\bar{\alpha}_{1}\right)\left(\alpha_{3}+\bar{\alpha}_{2}\right)}\right)^{2} .
$$

Step 3: Other velocities. On curves $x+\beta t=c$ with $\beta \in \mathbb{R} \backslash\left\{\operatorname{Im}\left(\alpha_{1}\right), \operatorname{Im}\left(\alpha_{3}\right)\right\}$, one checks $q=1-P / p \rightarrow 0$ for $t \rightarrow-\infty$, showing that there is no asymptotic contribution.

\section{Behavior for $t \rightarrow \infty$.}

Step 1: Moving With the two solitons of EQUAL Velocity. On curves $x+2 \operatorname{Im}\left(\alpha_{1}\right) t=c$, we have $1 /\left|\ell_{3}(x, t)\right| \rightarrow 0$ for $t \rightarrow \infty$. Here we divide in both determinants $P, p$ the third row by $\ell_{3}$ and the sixth row by $\bar{\ell}_{3}$. Focussing on $P$, we get $P \rightarrow P_{0}$, where

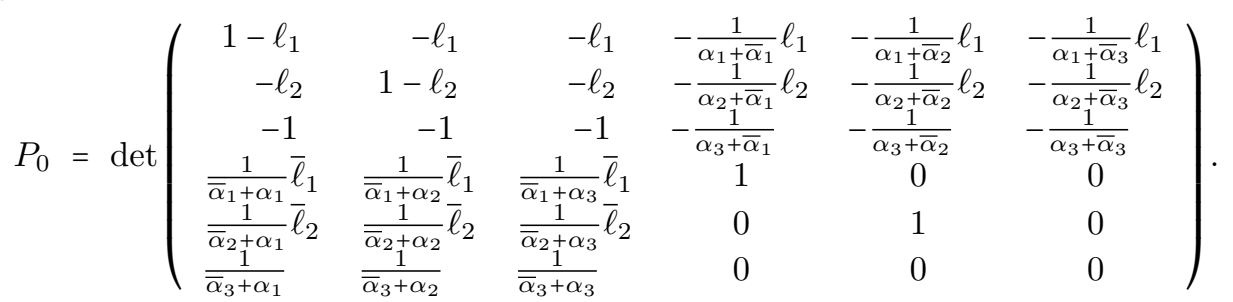

To identify the solution, we 
(1) multiply the third row by $\left(\alpha_{3}+\bar{\alpha}_{3}\right) /\left(\alpha_{i}+\bar{\alpha}_{3}\right) \ell_{i}$ and subtract it from the $i$-th row $(i=1,2)$,

(2) multiply the third column by $\left(\bar{\alpha}_{3}+\alpha_{3}\right) /\left(\bar{\alpha}_{3}+\alpha_{j}\right)$ and subtract it from the $j$-th column $(j=1,2)$.

After expansion, we get

$$
P_{0}=\left(\frac{1}{\alpha_{3}+\bar{\alpha}_{3}}\right)^{2} \operatorname{det}(I+T)
$$

with

$$
T=\left(\begin{array}{cccc}
-\gamma_{13} f_{1} & -\gamma_{23} f_{1} & -\bar{\gamma}_{13} \frac{1}{\alpha_{1}+\bar{\alpha}_{1}} f_{1} & -\bar{\gamma}_{23} \frac{1}{\alpha_{1}+\bar{\alpha}_{2}} f_{1} \\
-\gamma_{13} f_{2} & -\gamma_{23} f_{2} & -\bar{\gamma}_{13} \frac{1}{\alpha_{2}+\bar{\alpha}_{1}} f_{2} & -\bar{\gamma}_{23} \frac{1}{\alpha_{2}+\bar{\alpha}_{2}} f_{2} \\
\gamma_{13} \frac{1}{\bar{\alpha}_{1}+\alpha_{1}} \bar{f}_{1} & \gamma_{23} \frac{1}{\bar{\alpha}_{1}+\alpha_{2}} \bar{f}_{1} & 0 & 0 \\
\gamma_{13} \frac{1}{\bar{\alpha}_{2}+\alpha_{1}} \bar{f}_{2} & \gamma_{23} \frac{1}{\bar{\alpha}_{2}+\alpha_{2}} \bar{f}_{2} & 0 & 0
\end{array}\right),
$$

and $f_{i}=\gamma_{i 3} \ell_{i}$ for $i=1,2\left(\right.$ recall $\left.\gamma_{i j}=\left(\alpha_{i}-\alpha_{j}\right) /\left(\alpha_{i}+\bar{\alpha}_{j}\right)\right)$. Factorizing $T=T_{1} T_{2}$, where

$T_{1}=\left(\begin{array}{cccc}-f_{1} & -f_{1} & -\frac{1}{\alpha_{1}+\bar{\alpha}_{1}} f_{1} & -\frac{1}{\alpha_{1}+\bar{\alpha}_{2}} f_{1} \\ -f_{2} & -f_{2} & -\frac{1}{\alpha_{2}+\bar{\alpha}_{1}} f_{2} & -\frac{1}{\alpha_{2}+\bar{\alpha}_{2}} f_{2} \\ \frac{1}{\bar{\alpha}_{1}+\alpha_{1}} \bar{f}_{1} & \frac{1}{\bar{\alpha}_{1}+\alpha_{2}} \bar{f}_{1} & 0 & 0 \\ \frac{1}{\bar{\alpha}_{2}+\alpha_{1}} \bar{f}_{2} & \frac{1}{\bar{\alpha}_{2}+\alpha_{2}} \bar{f}_{2} & 0 & 0\end{array}\right), T_{2}=\left(\begin{array}{cccc}\gamma_{13} & 0 & 0 & 0 \\ 0 & \gamma_{23} & 0 & 0 \\ 0 & 0 & \bar{\gamma}_{13} & 0 \\ 0 & 0 & 0 & \bar{\gamma}_{23}\end{array}\right)$

and using $\operatorname{det}\left(I+T_{1} T_{2}\right)=\operatorname{det}\left(I+T_{2} T_{1}\right)$, we find

$$
P_{0}=\left(\frac{1}{\alpha_{3}+\bar{\alpha}_{3}}\right)^{2} \operatorname{det}\left(\begin{array}{cccc}
1-g_{1} & -g_{1} & -\frac{1}{\alpha_{1}+\bar{\alpha}_{1}} g_{1} & -\frac{1}{\alpha_{1}+\bar{\alpha}_{2}} g_{1} \\
-g_{2} & 1-g_{2} & -\frac{1}{\alpha_{2}+\bar{\alpha}_{1}} g_{2} & -\frac{1}{\alpha_{2}+\bar{\alpha}_{2}} g_{2} \\
\frac{1}{\bar{\alpha}_{1}+\alpha_{1}} \bar{g}_{1} & \frac{1}{\bar{\alpha}_{1}+\alpha_{2}} \bar{g}_{1} & 1 & 0 \\
\overline{1}_{2}+\bar{g}_{2} & \overline{1}_{2} & 1 & 0 \\
\bar{\alpha}_{2}+\alpha_{2} & \bar{g}_{2} & 0 & 1
\end{array}\right)
$$

with $g_{i}=\gamma_{i 3}^{2} \ell_{i}$ for $i=1,2$.

The factor $\left(\alpha_{3}+\bar{\alpha}_{3}\right)^{-2}$ also appears in front of the other determinant and hence cancels out in the quotient. As a result we re-find the superposition of the first two solitons, but with the initial shifts $\varphi_{i}, i=1,2$, replaced by

$$
\psi_{i}=\varphi_{i}+\log \left(\frac{\alpha_{i}-\alpha_{3}}{\alpha_{i}+\bar{\alpha}_{3}}\right)^{2} .
$$

These formulas also show that the involved two solitons suffer different positionand phase-shifts from the collision with the third soliton.

Step 2: Moving With the Remaining Soliton. Consider curves $x+2 \operatorname{Im}\left(\alpha_{3}\right) t=$ $c$. Here $\left|\ell_{i}(x, t)\right| \rightarrow 0$ for $t \rightarrow \infty, i=1,2$, and

$$
P \rightarrow \operatorname{det}\left(\begin{array}{cc}
1-\ell_{3} & -\frac{1}{\alpha_{3}+\bar{\alpha}_{3}} \ell_{3} \\
\frac{1}{\bar{\alpha}_{3}+\alpha_{3}} \bar{\ell}_{3} & 1
\end{array}\right) .
$$

Thus, the third soliton re-emerges as obtained by using the $1 \times 1$-matrix $A=\left(\alpha_{3}\right)$ and $a=(1), c=\left(e^{\varphi_{3}}\right)$ in Theorem 2.3 . 


\begin{tabular}{|c|c|c|c|c|c|}
\hline & $\begin{array}{r}\text { number of } \\
\text { eigenvalues }\end{array}$ & eigenvalues & multiplicities & $\begin{array}{c}\text { vector encoding } \\
\text { initial shifts }\end{array}$ & plotted region \\
\hline \multirow{3}{*}{$\begin{array}{c}\text { Fig. } 8 . \\
\text { left }\end{array}$} & \multirow[t]{3}{*}{$N=3$} & $\alpha_{1}=1$ & $n_{1}=1$ & $b_{1}=10$ & $-5 \leq x \leq 5$ \\
\hline & & $\alpha_{2}=2$ & $n_{2}=1$ & $b_{2}=36$ & $0 \leq t \leq 5$ \\
\hline & & $\alpha_{3}=4$ & $n_{3}=1$ & $b_{2}=40$ & \\
\hline \multirow{3}{*}{$\begin{array}{l}\text { Fig. } 8 \\
\text { middle }\end{array}$} & \multirow[t]{3}{*}{$N=3$} & $\alpha_{1}=1$ & $n_{1}=1$ & $b_{1}=10$ & $-5 \leq x \leq 5$ \\
\hline & & $\alpha_{2}=2$ & $n_{2}=1$ & $b_{2}=36$ & $0 \leq t \leq 5$ \\
\hline & & $\alpha_{3}=4$ & $n_{3}=1$ & $b_{2}=0.4$ & \\
\hline \multirow{3}{*}{$\begin{array}{c}\text { Fig. } 8 \\
\text { right }\end{array}$} & \multirow[t]{3}{*}{$N=3$} & $\alpha_{1}=1$ & $n_{1}=1$ & $b_{1}=50$ & $-5 \leq x \leq 5$ \\
\hline & & $\alpha_{2}=2$ & $n_{2}=1$ & $b_{2}=36$ & $0 \leq t \leq 5$ \\
\hline & & $\alpha_{3}=4$ & $n_{3}=1$ & $b_{2}=0.4$ & \\
\hline \multirow[t]{3}{*}{ Fig. 9 } & \multirow[t]{3}{*}{$N=3$} & $\alpha_{1}=2$ & $n_{1}=1$ & $b_{1}=60$ & $-5 \leq x \leq 5$ \\
\hline & & $\alpha_{2}=3$ & $n_{2}=1$ & $b_{2}=210$ & $0 \leq t \leq 5$ \\
\hline & & $\alpha_{3}=4$ & $n_{3}=1$ & $b_{2}=168$ & \\
\hline \multirow{2}{*}{$\begin{array}{c}\text { Fig. } 10 \\
\text { left }\end{array}$} & \multirow[t]{2}{*}{$N=2$} & $\alpha_{1}=2$ & $n_{1}=1$ & $b_{1}=36$ & $-5 \leq x \leq 5$ \\
\hline & & $\alpha_{2}=4$ & $n_{2}=2$ & $b_{2}=(-16,192)$ & $-3 \leq t \leq 3$ \\
\hline \multirow{2}{*}{$\begin{array}{c}\text { Fig. } 10 \\
\text { right }\end{array}$} & \multirow[t]{2}{*}{$N=2$} & $\alpha_{1}=2$ & $n_{1}=2$ & $b_{1}=(3,144) e^{-4}$ & $-6 \leq x \leq 8$ \\
\hline & & $\alpha_{2}=4$ & $n_{2}=2$ & $b_{2}=(480,576)$ & $-5 \leq t \leq 5$ \\
\hline
\end{tabular}

TABLE 1. Summary of the data for the stationary wave packets of higher order in Figs. 8- 10.

Step 3: Other Velocities. As for $t \rightarrow \infty$, there is no asymptotic contribution on curves $x+\beta t=c$ with $\beta \in \mathbb{R} \backslash\left\{\operatorname{Im}\left(\alpha_{1}\right), \operatorname{Im}\left(\alpha_{3}\right)\right\}$.

Using arguments like in Section 8, the above sketch can be complemented to prove uniform convergence. However, it may be more interesting to explore the asymptotics for the general case.

Selected computer graphics. Superpositions of wave packets with the same velocity can lead to bizarre structures. Fig. 8 illustrates this for three stationary solitons which gradually are positioned closer to each other. Whereas the solitons appear well-separated on the left-hand side, they become unrecognizable when plotted with a common geometric center as in the plot on the right-hand side. In Fig. 9, again three stationary solitons with the same geometric center are plotted, the strikingly different pattern comes from changing one of the eigenvalues: whereas $\alpha_{1}=1$, $\alpha_{2}=2, \alpha_{3}=4$ in Figure 8, we have $\alpha_{1}=2, \alpha_{2}=3, \alpha_{3}=4$ in Figure 9 .

For two further illustrations involving superpositions of stationary wave packets of higher order see Fig. 10 . 

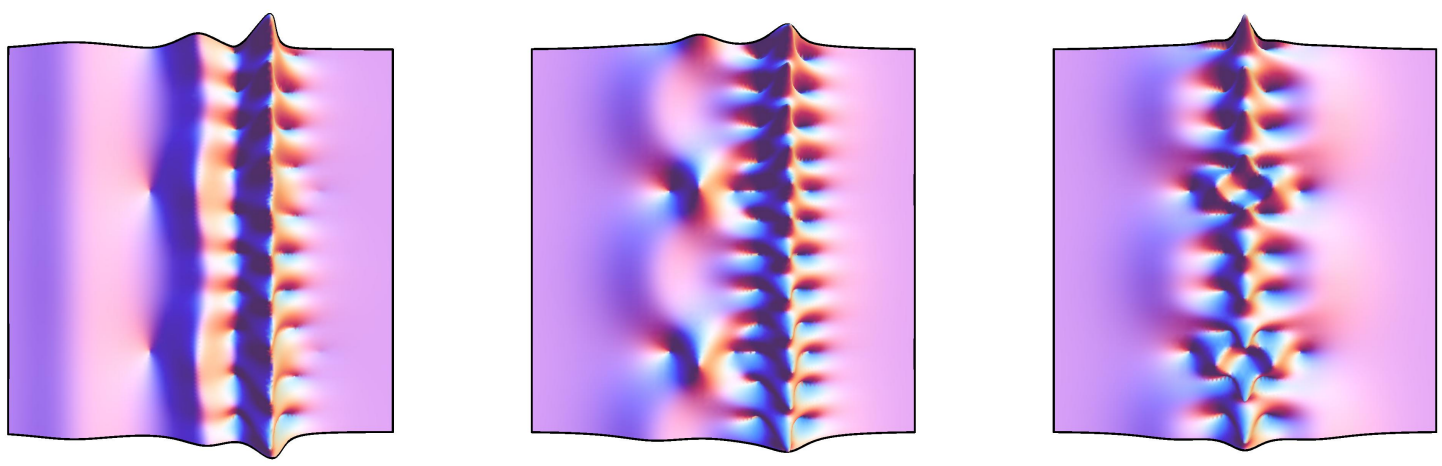

FiguRE 8. Three stationary solitons for different choices of initial position-shifts, see Table 1 for details. Note that in the plot on the right-hand side the solitons have the same geometric center.

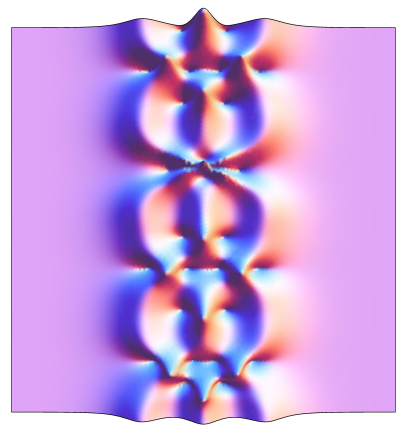

Figure 9. Three stationary solitons, all with the same geometric center. In contrast to the plot on the right-hand side of Fig. 8, one of the eigenvalues has been changed.

\section{Appendix A. Selected computer graphics}

In this appendix we provide some illustrations for MPS's of higher complexity. Table 2 displays the data used for the respective plots in Figures 11 - 14. As often before, all plots show the modulus of the respective solution. These plots are produced by a Mathematica program directly based on Proposition 7.1 .

\section{Appendix B. Eigenvalue Relations in the solution formula}

The aim of this appendix is to explain how to replace eigenvalues $\alpha$ of the matrix $A$ in Theorem 2.3 by $-\bar{\alpha}$ (see also [10, p.26] for a similar symmetry property in the pure soliton case). 

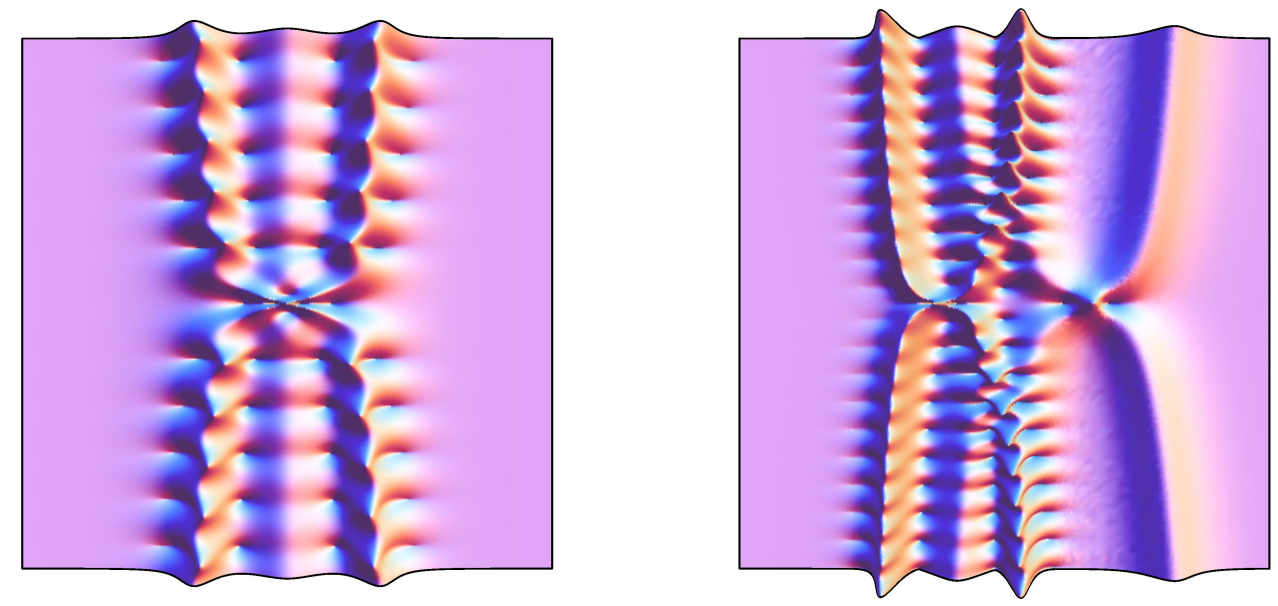

Figure 10. The plot to the left shows a stationary soliton and a stationary wave packet consisting of two solitons, the initial position-shifts chosen in such a way that their geometric centers coincide. The plot to the right shows two stationary wave packets, both consisting of two solitons but with different geometric center.
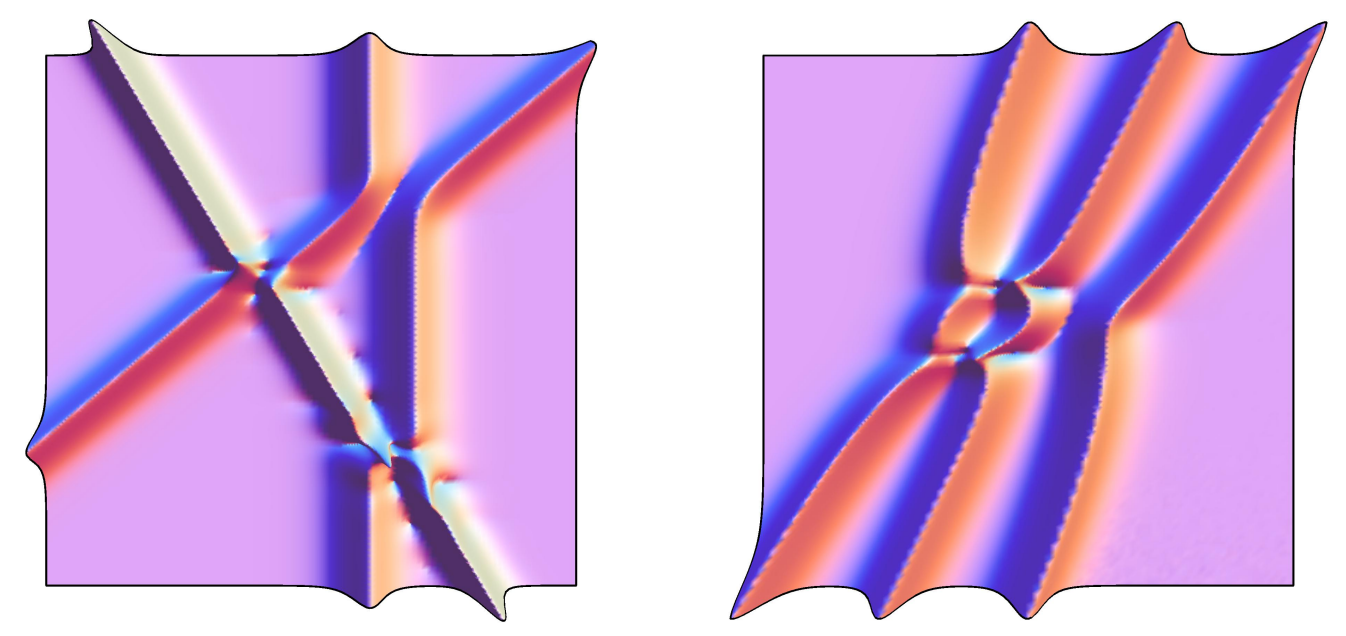

FiguRe 11. A 3-soliton versus a wave packet consisting of three solitons, see Table 2 for details. 

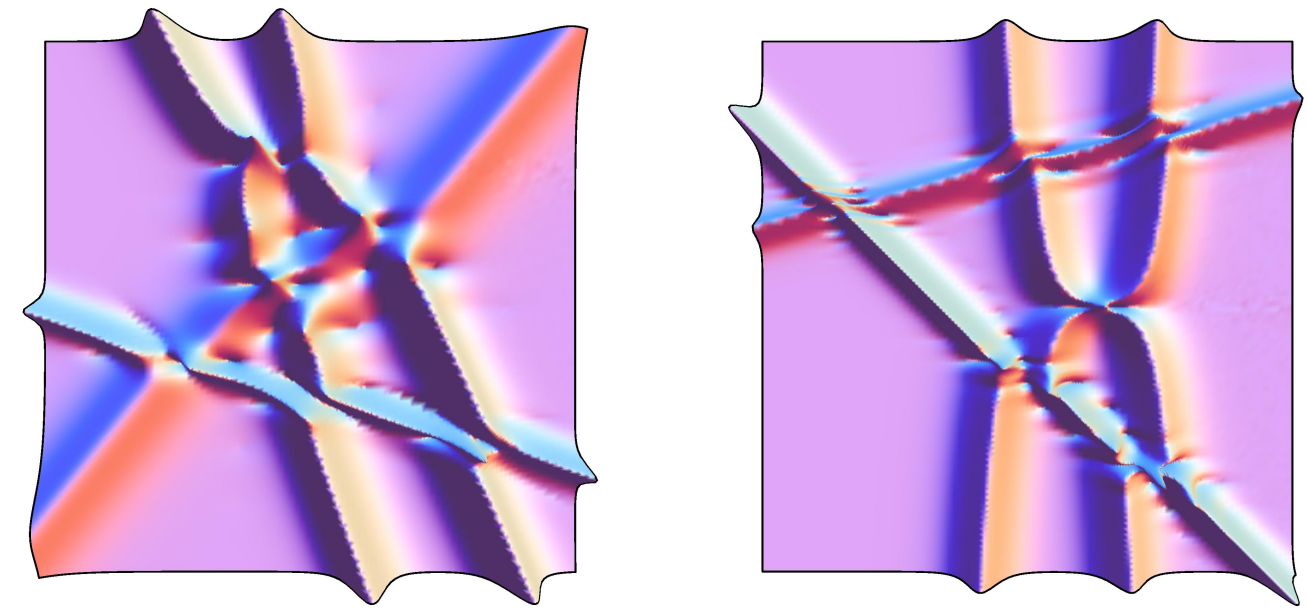

Figure 12. Collisions of two solitons with a wave packet consisting of two solitons.
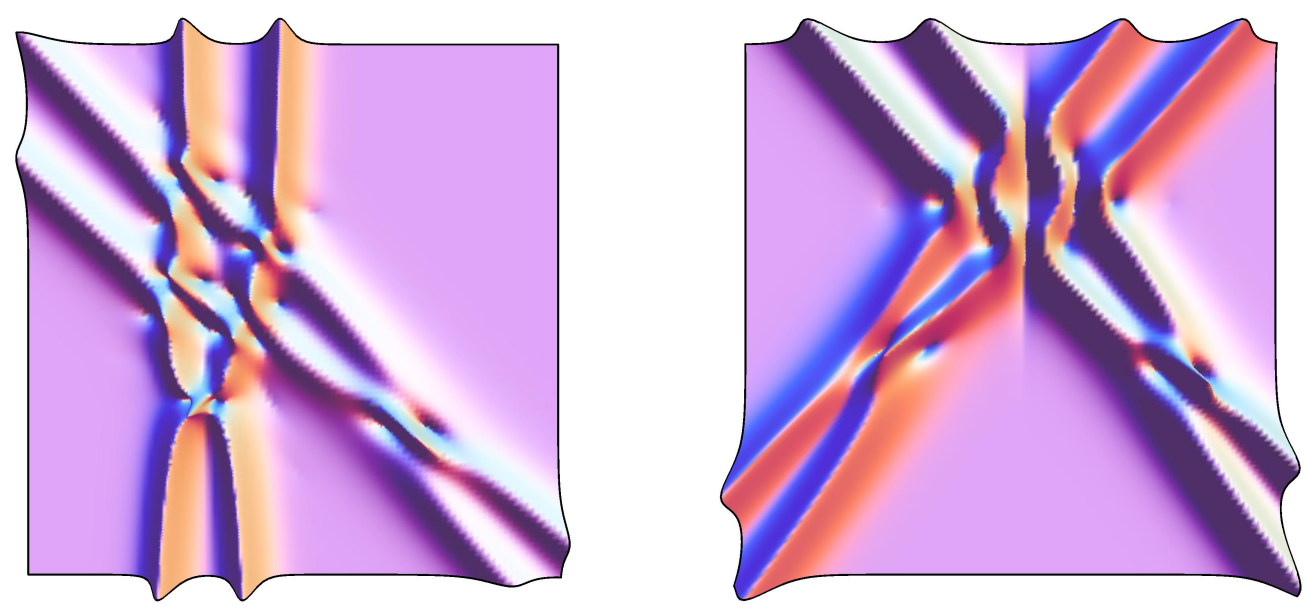

Figure 13. Collisions of two wave packets, both consisting of two solitons.

Lemma B.1. Let Assumptions 3.1 and 3.2 be satisfied, and let $A_{k_{1}}, A_{k_{2}}$ be Jordan blocks with the same eigenvalue $\alpha_{k_{1}}=\alpha_{k_{2}}$ and with size $n_{k_{1}} \leq n_{k_{2}}$. Then the solution given in Theorem 2.3 is not altered if we simultaneously replace $A$ by $\widetilde{A}$ and $a, c$ by $\widetilde{a}, \widetilde{c}$, where

a) $\widetilde{A}$ is the matrix obtained from $A$ by deleting the block $A_{k_{1}}$, 

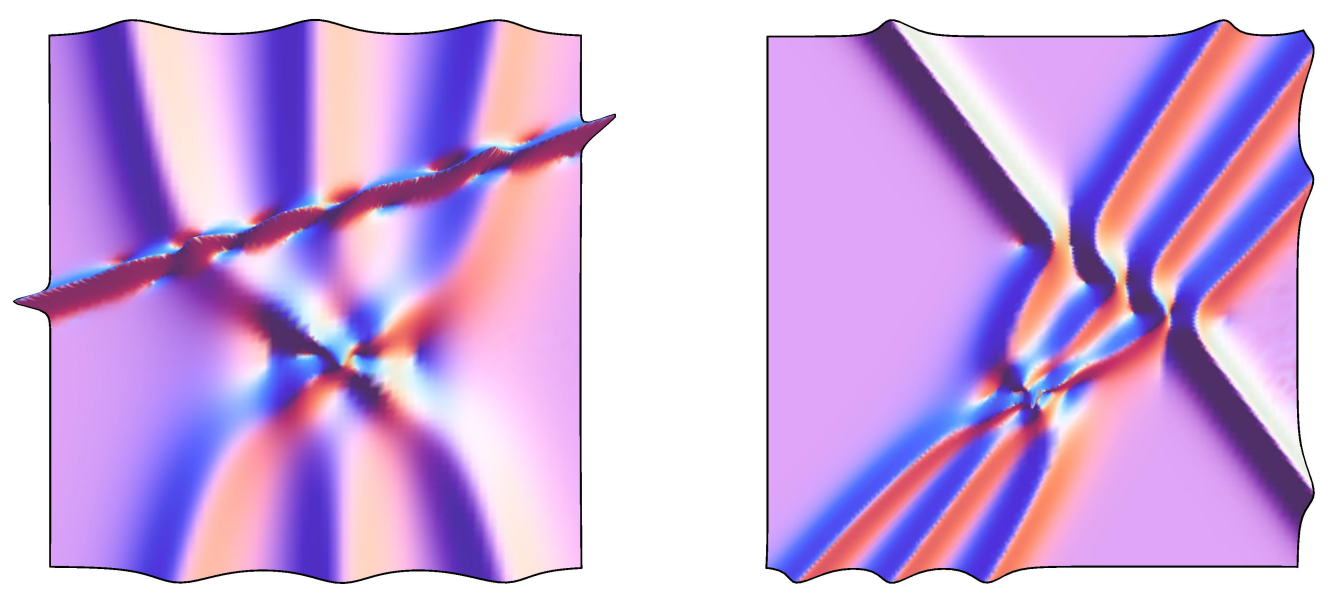

FIGURE 14. Collisions of a soliton with a wave packet consisting of three solitons.

b) $\widetilde{a}, \widetilde{c}$ are vectors obtained from a, c by replacing $a_{k_{2}}, c_{k_{2}}$ by $\widetilde{a}_{k_{2}}, \widetilde{c}_{k_{2}}$ appropriately and deleting $a_{k_{1}}, c_{k_{1}}$.

In the proof we will specify how to choose $\widetilde{a}_{k_{2}}, \widetilde{c}_{k_{2}}$.

Proof. We may assume $k_{1}=1, k_{2}=2$, and that the solution is in reduced form, see Proposition 7.1. Define the $n_{1} \times n_{2}$-matrix $\Pi$, the $\left(n_{1}+n_{2}\right) \times n_{2}$-matrix $\Phi$, and the $n \times\left(n-n_{1}\right)$-matrix $\Psi$ by

$$
\Pi=\left(\begin{array}{ll}
I_{n_{1}} & 0
\end{array}\right), \quad \Phi=\left(\begin{array}{c}
\Pi \\
I_{n_{2}}
\end{array}\right), \quad \Psi=\left(\begin{array}{cc}
\Phi & 0 \\
0 & I_{n-n_{1}}
\end{array}\right) .
$$

For the $T_{i j}$ as in Proposition 7.1 we observe that $T_{1 j}=\Pi T_{2 j}$ for $j \geq 2, T_{i 1}=T_{i 2} \Pi^{t r}$ for $i \geq 2$, and $T_{11}=\Pi T_{22} \Pi^{t r}$. Hence

$$
\left(\begin{array}{ll}
T_{11} & T_{12} \\
T_{21} & T_{22}
\end{array}\right)=\left(\begin{array}{cc}
\Pi T_{22} \Pi^{t r} & \Pi T_{22} \\
T_{22} \Pi^{t r} & T_{22}
\end{array}\right)=\Phi T_{22} \Phi^{t r}
$$

and

$$
\left(\begin{array}{c}
T_{1 j} \\
T_{2 j}
\end{array}\right)=\left(\begin{array}{c}
\Pi T_{2 j} \\
T_{2 j}
\end{array}\right)=\Phi T_{2 j}, \quad\left(\begin{array}{ll}
T_{i 1} & T_{i 2}
\end{array}\right)=\left(\begin{array}{c}
T_{i 2} \Pi^{t r} \\
T_{i 2}
\end{array}\right)=T_{i 2} \Phi^{t r}
$$

for $i, j \geq 2$. This shows $T=\Psi \widehat{T} \Psi^{t r}$, where $\widehat{T}=\left(T_{i j}\right)_{i, j=2}^{N}$, and similarly one obtains $f f^{t r}=\Psi \widehat{f}^{t r} \Psi^{t r}$, where $\widehat{f}=\left(e_{n_{j}}^{(1)}\right)_{j=2}^{N}$. As a result,

$$
\begin{aligned}
P & =\operatorname{det}\left(I+\left(\begin{array}{cc}
M & 0 \\
0 & \bar{M}
\end{array}\right)\left(\begin{array}{cc}
\Psi & 0 \\
0 & \Psi
\end{array}\right)\left(\begin{array}{cc}
-\widehat{f f}^{t r} & -\widehat{T} \\
\widehat{\widehat{T}} & 0
\end{array}\right)\left(\begin{array}{cc}
\Psi^{t r} & 0 \\
0 & \Psi^{t r}
\end{array}\right)\right) \\
& =\operatorname{det}\left(I+\left(\begin{array}{cc}
\widehat{M} & 0 \\
0 & \widehat{\bar{M}}
\end{array}\right)\left(\begin{array}{cc}
-\widehat{f f}^{t r} & -\widehat{T} \\
\widehat{\widehat{T}} & 0
\end{array}\right)\right),
\end{aligned}
$$




\begin{tabular}{|c|c|c|c|c|c|}
\hline \multicolumn{1}{|c|}{$\begin{array}{c}\text { number of } \\
\text { eigenvalues }\end{array}$} & eigenvalues & multiplicities & $\begin{array}{c}\text { vector encoding } \\
\text { initial shifts }\end{array}$ & plotted region \\
\hline $\begin{array}{c}\text { Fig. 11 } \\
\text { left }\end{array}$ & $N=3$ & $\alpha_{1}=1$ & $n_{1}=1$ & $b_{1}=2 e^{-1}$ & $-20 \leq x \leq 20$ \\
& & $\alpha_{2}=1.5+0.2 \mathrm{i}$ & $n_{2}=1$ & $b_{2}=3 e^{10}$ & $-40 \leq t \leq 20$ \\
& $\alpha_{3}=0.9-0.4 \mathrm{i}$ & $n_{3}=1$ & $b_{3}=4$ & \\
\hline $\begin{array}{c}\text { Fig. 11 } \\
\text { right }\end{array}$ & $N=3$ & $\alpha_{1}=1-0.1 \mathrm{i}$ & $n_{1}=3$ & $b_{1}=(600,12,8)$ & $-20 \leq x \leq 20$ \\
& & & & & $-50 \leq t \leq 50$ \\
\hline $\begin{array}{c}\text { Fig. 12 } \\
\text { left }\end{array}$ & $N=3$ & $\alpha_{1}=0.7+0.7 \mathrm{i}$ & $n_{1}=1$ & $b_{1}=3 e^{30}$ & $-15 \leq x \leq 25$ \\
& & $\alpha_{2}=0.5-0.2 \mathrm{i}$ & $n_{2}=1$ & $b_{2}=e^{-3}$ & $-60 \leq t \leq 20$ \\
\hline $\begin{array}{c}\text { Fig. 12 } \\
\text { right }\end{array}$ & $N=3$ & $\alpha_{1}=0.5-0.7 \mathrm{i}$ & $n_{1}=1$ & $b_{3}=(0,2)$ & $-20 \leq x \leq 20$ \\
& & $\alpha_{2}=1.5+0.2 \mathrm{i}$ & $n_{2}=1$ & $b_{2}=3 e^{10}$ & $-50 \leq t \leq 50$ \\
\hline $\begin{array}{c}\text { Fig. } 13 \\
\text { left }\end{array}$ & $N=2$ & $\alpha_{1}=1$ & $n_{1}=2$ & $b_{1}=(6,1) e^{6}$ & $-25 \leq x \leq 35$ \\
\hline $\begin{array}{c}\text { Fig. } 13 \\
\text { right }\end{array}$ & $N=2$ & $\alpha_{1}=0.5+0.4 \mathrm{i}$ & $n_{1}=2$ & $b_{1}=(-20,1) e^{-10}$ & $-30 \leq x \leq 40$ \\
\hline $\begin{array}{c}\text { Fig. 14 } \\
\text { left }\end{array}$ & $N=2$ & $\alpha_{2}=0.5-0.4 \mathrm{i}$ & $n_{2}=2$ & $b_{2}=(10,1) e^{5}$ & $-30 \leq t \leq 40$ \\
\hline $\begin{array}{c}\text { Fig. } 14 \\
\text { right }\end{array}$ & $N=2$ & $\alpha_{1}=1-\mathrm{i}$ & $n_{2}=1$ & $b_{1}=2 e^{30}$ & $-20 \leq x \leq 20$ \\
\hline
\end{tabular}

TABle 2. Summary of the data for the visualizations in Fig. 11, 14.

where $\widehat{M}=\Psi^{\operatorname{tr}} M \Psi$. Furthermore, we get $\widehat{M}=\operatorname{diag}\left\{\widehat{M}_{2}, M_{3}, \ldots, M_{N}\right\}$, and $\widehat{M}_{2}=$ $\Phi^{t r} \operatorname{diag}\left\{M_{1}, M_{2}\right\} \Phi=M_{2}+\Pi^{t r} M_{1} \Pi$.

Setting $b_{1}^{(\mu)}=0$ for $\mu>n_{1}$, we can write

$$
\widehat{M}_{2}=\left(\begin{array}{ccc}
\widehat{m}_{2}^{(1)} & & \widehat{m}_{2}^{\left(n_{2}\right)} \\
\widehat{m}_{2}^{\left(n_{2}\right)} & &
\end{array}\right)
$$

for $\widehat{m}_{2}^{(\mu)}=\sum_{\kappa=1}^{n_{2}-(\mu-1)}\left(b_{1}^{(\mu-1+\kappa)}+b_{2}^{(\mu-1+\kappa)}\right) \frac{1}{(\kappa-1) !} \frac{\partial^{\kappa-1}}{\partial \alpha^{\kappa-1}} e^{\alpha x-\mathrm{i} \alpha^{2} t}$.

The analogous reformulation holds for $p$. This shows that $q=1-P / p$ is not altered if we delete the rows/columns corresponding to the first block and, simultaneously, replace $b_{2}^{(\mu)}$ by $\widetilde{b}_{2}^{(\mu)}=b_{1}^{(\mu)}+b_{2}^{(\mu)}$ for $\mu \leq n_{1}$.

Finally we explain how to choose $\widetilde{a}_{2}, \widetilde{c}_{2}$ appropriately. Set $\widetilde{a}_{2}^{(\mu)}=1$ for $\mu \leq n_{2}$ and $\widetilde{c}_{2}^{(\mu)}=\widetilde{b}_{2}^{(\mu)}-\widetilde{b}_{2}^{(\mu+1)}$ for $\mu<n_{2}, \widetilde{c}_{2}^{\left(n_{2}\right)}=\widetilde{b}_{2}^{\left(n_{2}\right)}$. Then

$$
\sum_{\kappa=1}^{n_{2}-\mu+1} \widetilde{a}_{2}^{(\kappa)} \widetilde{c}_{2}^{(\kappa+\mu-1)}=\sum_{\lambda=\mu}^{n_{2}} \widetilde{c}_{2}^{(\lambda)}=\widetilde{b}_{2}^{(\mu)},
$$

in other words $\widetilde{a}_{2}, \widetilde{c}_{2}$ are related to $\widetilde{b}_{2}$ as in Proposition 7.1 
Lemma B.1 tells that we can assume that the eigenvalues of $A$ have geometric multiplicity 1, i.e. each eigenvalue appears in exactly one Jordan block.

Proposition B.2. Let Assumptions 3.1 and 3.2 be satisfied and let $\widetilde{A}$ be the matrix obtained from $A$ by replacing an eigenvalue $\alpha$ of geometric multiplicity 1 by $-\bar{\alpha}$. Then there are vectors $\widetilde{a}, \widetilde{c}$ such that the solution given in Theorem 2.3 is not altered if we replace simultaneously $A$ by $\widetilde{A}$, and $a, c$ by $\widetilde{a}, \widetilde{c}$.

Note that the proof yields concrete formulas for $\widetilde{a}, \widetilde{c}$.

Proof. We may assume $\alpha=\alpha_{1}$. Recall that $A_{1}, \ldots, A_{N}$ denote the Jordan blocks of $A$, and write

$$
A=\left(\begin{array}{cc}
A_{1} & 0 \\
0 & \widehat{A}_{2}
\end{array}\right), \quad a=\left(\begin{array}{c}
a_{1} \\
\widehat{a}_{2}
\end{array}\right), \quad c=\left(\begin{array}{c}
c_{1} \\
\widehat{c}_{2}
\end{array}\right)
$$

for $\widehat{A}_{2}=\operatorname{diag}\left\{A_{2}, \ldots, A_{N}\right\}$, and $\widehat{a}_{2}=\left(a_{j}\right)_{j=2}^{N}, \widehat{c_{2}}=\left(c_{j}\right)_{j=2}^{N}$. To simplify notation, we will again write $A_{2}, a_{2}, c_{2}$ for $\widehat{A}_{2}, \widehat{a}_{2}, \widehat{c}_{2}$.

Let $C$ be the solution of the Sylvester equation $A X+X \bar{A}=c \bar{a}^{t r}$. Then we have $C=\left(C_{i j}\right)_{i, j=1}^{2}$ with

$$
A_{i} C_{i j}+C_{i j} \bar{A}_{j}=c_{i} \bar{a}_{j}^{t r}
$$

Observe that $C_{11}$ is invertible by Lemma 7.3, Assumption 3.2 and 24, Theorem 4.1].

Set $\Theta_{1}=\operatorname{diag}\left\{(-1)^{\mu-1} \mid \mu=1, \ldots, n_{1}\right\}$. Then we can write $\widetilde{A}=\operatorname{diag}\left\{\widetilde{A}_{1}, \widetilde{A}_{2}\right\}$ for $\widetilde{A_{1}}=-\Theta_{1} \bar{A}_{1} \Theta_{1}$ and $\widetilde{A}_{2}=A_{2}$. Furthermore, we define

$$
\widetilde{a}=\left(\begin{array}{c}
\widetilde{a}_{1} \\
\widetilde{a}_{2}
\end{array}\right)=\left(\begin{array}{c}
\Theta_{1}\left(\bar{C}_{11}^{-1}\right)^{t r} a_{1} \\
a_{2}-\bar{C}_{12}^{t r}\left(\bar{C}_{11}^{-1}\right)^{t r} a_{1}
\end{array}\right), \quad \widetilde{c}=\left(\begin{array}{c}
\widetilde{c}_{1} \\
\widetilde{c}_{2}
\end{array}\right)=\left(\begin{array}{c}
\Theta_{1} C_{11}^{-1} c_{1} \\
c_{2}-C_{21} C_{11}^{-1} c_{1}
\end{array}\right) .
$$

Note that $0 \notin \operatorname{spec}(A)+\operatorname{spec}(\bar{A})$ implies $0 \notin \operatorname{spec}(\widetilde{A})+\operatorname{spec}(\widetilde{A})$.

Now the proof proceeds in two steps. First we solve the Sylvester equation corresponding to $\widetilde{A}, \widetilde{a}, \widetilde{c}$, then we show these replacements do not alter the solution.

Step 1. It is straightforward to verify that

$$
\widetilde{C}=\left(\begin{array}{ll}
\widetilde{C}_{11} & \widetilde{C}_{12} \\
\widetilde{C}_{21} & \widetilde{C}_{22}
\end{array}\right)=\left(\begin{array}{cc}
-\Theta_{1} C_{11}^{-1} \Theta_{1} & \Theta_{1} C_{11}^{-1} C_{12} \\
C_{21} C_{11}^{-1} \Theta_{1} & C_{22}-C_{21} C_{11}^{-1} C_{12}
\end{array}\right)
$$

satisfies $\widetilde{A} \widetilde{C}+\widetilde{C} \overline{\widetilde{A}}=\widetilde{\bar{c}}^{t r}$. Exemplarily we treat the lower left corner:

$$
\begin{aligned}
\widetilde{c}_{2} \overline{\bar{a}}_{1}^{t r}= & \left(c_{2}-C_{21} C_{11}^{-1} c_{1}\right) \bar{a}_{1}^{t r} C_{11}^{-1} \Theta_{1} \\
& =\left(\left(A_{2} C_{21}+C_{21} \bar{A}_{1}\right)-C_{21} C_{11}^{-1}\left(A_{1} C_{11}+C_{11} \bar{A}_{1}\right)\right) C_{11}^{-1} \Theta_{1} \\
& =A_{2} C_{21} C_{11}^{-1} \Theta_{1}-C_{21} C_{11}^{-1} A_{1} \Theta_{1} \\
& =A_{2}\left(C_{21} C_{11}^{-1} \Theta_{1}\right)+\left(C_{21} C_{11}^{-1} \Theta_{1}\right)\left(-\Theta_{1} A_{1} \Theta_{1}\right) \\
& =\widetilde{A}_{2} \widetilde{C}_{21}+\widetilde{C}_{21} \overline{\widetilde{A}}_{1} .
\end{aligned}
$$

Step 2. Let $F=\operatorname{diag}\left\{F_{1}, F_{2}\right\}$ with $F_{j}(x, t)=\exp \left(A_{j} x-\mathrm{i} A_{j}^{2} t\right)$ and $\widetilde{F}=\operatorname{diag}\left\{\widetilde{F}_{1}, \widetilde{F}_{2}\right\}$ with $\widetilde{F}_{j}(x, t)=\exp \left(\widetilde{A}_{j} x-\mathrm{i} \widetilde{A}_{j}^{2} t\right)$. Then

$$
\begin{aligned}
\widetilde{F}_{1} & =\exp \left(\widetilde{A}_{1} x-\mathrm{i} \widetilde{A}_{1}^{2} t\right)=\exp \left(\left(-\Theta_{1} \bar{A}_{1} \Theta_{1}\right) x-\mathrm{i}\left(-\Theta_{1} \bar{A}_{1} \Theta_{1}\right)^{2} t\right) \\
& =\exp \left(-\Theta_{1}\left(\bar{A}_{1} x+\mathrm{i} \bar{A}_{1}^{2} t\right) \Theta_{1}\right)=\Theta_{1} \exp \left(-\left(\overline{A_{1} x-\mathrm{i} A_{1}^{2} t}\right)\right) \Theta_{1} \\
& =\Theta_{1} \bar{F}_{1}^{-1} \Theta_{1} .
\end{aligned}
$$


Hence we can factorize

$$
\widetilde{F}=\Theta \bar{M}_{1}^{-1} M_{2} \Theta
$$

for $M_{1}=\left(\begin{array}{cc}F_{1} & 0 \\ 0 & I\end{array}\right), M_{2}=\left(\begin{array}{cc}I & 0 \\ 0 & F_{2}\end{array}\right), \Theta=\left(\begin{array}{cc}\Theta_{1} & 0 \\ 0 & I\end{array}\right)$. Moreover, for $D=\left(\begin{array}{cc}-I & 0 \\ C_{21} & C_{22}\end{array}\right)$, and $S=\left(\begin{array}{cc}C_{11} & C_{12} \\ 0 & I\end{array}\right)$ (with $S^{-1}=\left(\begin{array}{cc}C_{11}^{-1} & -C_{11}^{-1} C_{12} \\ 0 & I\end{array}\right)$, we can write

$$
\widetilde{a}=\Theta\left(\bar{S}^{-1}\right)^{\operatorname{tr}} a, \quad \widetilde{C}=\Theta D S^{-1} \Theta .
$$

Using these factorization, we get

$$
\begin{aligned}
& \left(\begin{array}{cc}
I-\widetilde{F} \widetilde{c}^{t r} & -\widetilde{F} \widetilde{C} \\
\widetilde{F} \widetilde{C} & I
\end{array}\right) \\
& =\left(\begin{array}{cc}
I-\Theta \bar{M}_{1}^{-1} M_{2} \Theta \widetilde{c} a^{t r} \bar{S}^{-1} \Theta & -\Theta \bar{M}_{1}^{-1} M_{2} D S^{-1} \Theta \\
\Theta M_{1}^{-1} \bar{M}_{2} \bar{D} \bar{S}^{-1} \Theta & I
\end{array}\right) \\
& =\left(\begin{array}{cc}
\Theta \bar{M}_{1}^{-1} & 0 \\
0 & \Theta M_{1}^{-1}
\end{array}\right)\left(\begin{array}{cc}
\bar{M}_{1} \bar{S}_{-} M_{2} \Theta \widetilde{c} a^{t r} & -M_{2} D \\
\bar{M}_{2} \bar{D} & M_{1} S
\end{array}\right)\left(\begin{array}{cc}
\bar{S}^{-1} \Theta & 0 \\
0 & S^{-1} \Theta
\end{array}\right) \text {. }
\end{aligned}
$$

yielding

$$
\operatorname{det}\left(\begin{array}{cc}
I-\widetilde{F} \widetilde{F} \widetilde{c}^{t r} & -\widetilde{F} \widetilde{C} \\
\widetilde{F} \widetilde{C} & I
\end{array}\right)=\gamma^{-1} \operatorname{det}\left(\begin{array}{cc}
\bar{M}_{1} \bar{S}-M_{2} \Theta \widetilde{c} a^{t r} & -M_{2} D \\
\bar{M}_{2} \bar{D} & M_{1} S
\end{array}\right)
$$

with $\gamma=\left|\operatorname{det}(S) \operatorname{det}\left(M_{1}\right)\right|^{2}$.

Next set $T=\left(\begin{array}{cc}I & 0 \\ f a^{t r} & I\end{array}\right)$ with $f=\left(\begin{array}{c}C_{11}^{-1} c_{1} \\ 0\end{array}\right)$. Note that $\Theta \widetilde{c}+D f=\left(\begin{array}{c}0 \\ c_{2}\end{array}\right), S f=\left(\begin{array}{c}c_{1} \\ 0\end{array}\right)$. Thus

$$
\begin{gathered}
\left(\begin{array}{cc}
\bar{M}_{1} \bar{S}-M_{2} \Theta \widetilde{c} a^{t r} & -M_{2} D \\
\bar{M}_{2} D & M_{1} S
\end{array}\right) T=\left(\begin{array}{cc}
\bar{M}_{1} \bar{S}-M_{2}(\Theta \widetilde{c}+D f) a^{t r} & -M_{2} D \\
\bar{M}_{2} \bar{D}+M_{1} S f a^{t r} & M_{1} S
\end{array}\right) \\
=\left(\begin{array}{ccc}
\bar{M}_{1} \bar{S}-M_{2}\left(\begin{array}{c}
0 \\
c_{2}
\end{array}\right) a^{t r} & -M_{2} D \\
\bar{M}_{2} \bar{D}+M_{1}\left(\begin{array}{cc}
c_{1} \\
0
\end{array}\right) a^{t r} & M_{1} S
\end{array}\right) \\
=\left(\begin{array}{cccc}
\bar{F}_{1} \bar{C}_{11} & \bar{F}_{1} \bar{C}_{12} & I & 0 \\
-F_{2} c_{2} a_{1}^{t r} & I-F_{2} c_{2} a_{2}^{t r} & -F_{2} C_{21} & -F_{2} C_{22} \\
-I+F_{1} c_{1} a_{1}^{t r} & F_{1} c_{1} a_{2}^{t r} & F_{1} C_{11} & F_{1} C_{12} \\
\bar{F}_{2} \bar{C}_{21} & \bar{F}_{2} \bar{C}_{22} & 0 & I
\end{array}\right),
\end{gathered}
$$

which, after extracting $n_{1}$ times the factor -1 from the third row and then interchanging the first and the third row ( $n_{1}$ interchangings), gives

$$
\operatorname{det}\left(\begin{array}{cc}
\bar{M}_{1} \bar{S}-M_{2} \Theta \widetilde{c} a^{t r} & -M_{2} D \\
\bar{M}_{2} \bar{D} & M_{1} S
\end{array}\right)=\operatorname{det}\left(\begin{array}{cc}
I-F c a^{t r} & -F C \\
\bar{F} \bar{C} & I
\end{array}\right) .
$$

Using (B.2), B.3) we obtain

$$
P=\operatorname{det}\left(\begin{array}{cc}
I-F c a^{t r} & -F C \\
\bar{F} \bar{C} & I
\end{array}\right)=\gamma \operatorname{det}\left(\begin{array}{cc}
I-\widetilde{\widetilde{F}} \widetilde{c} a^{t r} & -\widetilde{F} \widetilde{C} \\
\widetilde{F} \widetilde{C} & I
\end{array}\right)
$$

showing that the replacement does change $P$ by the factor $\gamma$. Similar, somewhat easier manipulations allow to handle $p$, which also changes by the factor $\gamma$. Hence $q=1-P / p$ is not altered. 


\section{Appendix C. Global Regularity}

In this appendix we complete the proof of Theorem 2.3. Recall that we have already obtained a solution on $\Omega=\{(x, t) \mid I+L \bar{L}$ is invertible $\}$. Hence it suffices to show that $\operatorname{det}(I+L \bar{L}) \neq 0$ holds for every $(x, t) \in \mathbb{R}$. The main ingredient of the proof is the following factorization result for solutions of the Sylvester equation.

Proposition C.1. Let Assumptions 3.1 and 3.2 be met, and suppose $\operatorname{Re}\left(\alpha_{j}\right)>0$ for all $j$. Let $\varphi, \psi$ be polynomials of one complex variable $z$ satisfying

$$
\begin{aligned}
& \left.\frac{1}{(\nu-1) !} \frac{\partial^{\nu-1}}{\partial z^{\nu-1}}\right|_{z=\alpha_{j}} e^{\varphi(z)}=c_{j}^{\left(n_{j}-\nu+1\right)}, \\
& \left.\frac{1}{(\nu-1) !} \frac{\partial^{\nu-1}}{\partial z^{\nu-1}}\right|_{z=\alpha_{j}} e^{\psi(z)}=a_{j}^{(\nu)}
\end{aligned}
$$

for $\nu=1, \ldots, n_{j}, j=1, \ldots, N$. Moreover, set $\widetilde{\varphi}(z)=\overline{\varphi(\bar{z})}, \widetilde{\psi}(z)=\overline{\psi(\bar{z})}$.

Denote by $e_{j \nu} \in \mathbb{C}^{n}=\bigoplus_{j=1}^{N} \mathbb{C}^{n_{j}}$ the vector which is the $\nu$ th standard basis vector on the component $\mathbb{C}^{n_{j}}$ and the zero-vector on all other components. Define the operators $T: \mathbb{C}^{n} \longrightarrow L^{2}(-\infty, 0]$ and $S: L^{2}(-\infty, 0] \longrightarrow \mathbb{C}^{n}$ by

$$
T e_{j \nu}=f_{j \nu}, \quad S f=\sum_{j=1}^{N} \sum_{\nu=1}^{n_{j}} s_{j \nu}(f) e_{j \nu}
$$

where

$$
\begin{aligned}
f_{j \nu}(\sigma) & =\left.\frac{1}{(\nu-1) !} \frac{\partial^{\nu-1}}{\partial z^{\nu-1}}\right|_{z=\alpha_{j}} e^{z \sigma+\psi(z)}, \\
s_{j \nu}(f) & =\left.\int_{-\infty}^{0} f(s) \frac{1}{\left(n_{j}-\nu\right) !} \frac{\partial^{n_{j}-\nu}}{\partial z^{n_{j}-\nu}}\right|_{z=\alpha_{j}} e^{z s+\varphi(z)} d s,
\end{aligned}
$$

and the analogous operators $\widetilde{S}, \widetilde{T}$ which are obtained by replacing $\psi, \varphi$ with $\widetilde{\psi}, \widetilde{\varphi}$, respectively, and $\alpha_{j}$ with $\bar{\alpha}_{j}$.

Finally, let $C \in \mathcal{L}\left(\mathbb{C}^{n}\right)$ be the solution of the Sylvester equation $A X+X \bar{A}=c \bar{a}^{t r}$, and let $K \in \mathcal{L}\left(L^{2}(-\infty, 0]\right)$ be the integral operator $1^{3}$ with kernel

$$
k(\sigma, s)=\left.\sum_{j=1}^{N} \frac{1}{\left(n_{j}-1\right) !} \frac{\partial^{n_{j}-1}}{\partial z^{n_{j}-1}}\right|_{z=\alpha_{j}} e^{(\sigma+s) z+\varphi(z)+\psi(z)} .
$$

Then,

a) $K=T S$,

b) $C=S \widetilde{T}$,

c) $C \bar{C}$ is related to the integral operator $H$ with kernel

$$
h(\sigma, s)=\int_{-\infty}^{0} k(\sigma, \rho) \overline{k(\rho, s)} d \rho,
$$

which is self-adjoint and positive.

Note that Assumption 3.2 guarantees the existence of $\varphi$ and $\psi$.

\footnotetext{
3 i.e. $(K f)(\sigma)=\int_{-\infty}^{0} k(\sigma, s) f(s) d s$

${ }^{4}$ Two operators $S \in \mathcal{L}(E), T \in \mathcal{L}(F)$, where $E, F$ are complex Banach spaces, are called related if there exist $A \in \mathcal{L}(E, F)$ and $B \in \mathcal{L}(F, E)$ such that $S=B A$ and $T=A B$.
} 
Proof. In the sequel, we will consider variations of the integral kernels with respect to a parameter $z$ close to $\alpha_{j}$. Since $\operatorname{Re}\left(\alpha_{j}\right)>0$, we may assume $\operatorname{Re}(z) \geq \epsilon$ for some $\epsilon>0$.

a) Observe that

$$
s_{j \nu}(f)=\int_{-\infty}^{0} g_{j \nu}(s) f(s) d s \quad \text { for } \quad g_{j \nu}(s)=\left.\frac{1}{\left(n_{j}-\nu\right) !} \frac{\partial^{n_{j}-\nu}}{\partial z^{n_{j}-\nu}}\right|_{z=\alpha_{j}} e^{z s+\varphi(z)} .
$$

Hence,

$$
(T S f)(\sigma)=\sum_{j=1}^{N} \sum_{\nu=1}^{n_{j}} s_{j \nu}(f) f_{j \nu}(\sigma)=\int_{-\infty}^{0}\left(\sum_{j=1}^{N} \sum_{\nu=1}^{n_{j}} g_{j \nu}(s) f_{j \nu}(\sigma)\right) f(s) d s,
$$

showing that $T S$ is the integral operator with kernel

$$
\begin{aligned}
k & (\sigma, s)=\sum_{j=1}^{N} \sum_{\nu=1}^{n_{j}} g_{j \nu}(s) f_{j \nu}(\sigma) \\
& =\sum_{j=1}^{N} \frac{1}{\left(n_{j}-1\right) !} \sum_{\nu=1}^{n_{j}}\left(\begin{array}{c}
n_{j}-1 \\
\nu-1
\end{array}\right)\left(\left.\frac{\partial^{n_{j}-\nu}}{\partial z^{n_{j}-\nu}}\right|_{z=\alpha_{j}} e^{z s+\varphi(z)}\right)\left(\left.\frac{\partial^{\nu-1}}{\partial z^{\nu-1}}\right|_{z=\alpha_{j}} e^{z \sigma+\psi(z)}\right) \\
& =\left.\sum_{j=1}^{N} \frac{1}{\left(n_{j}-1\right) !} \frac{\partial^{n_{j}-1}}{\partial z^{n_{j}-1}}\right|_{z=\alpha_{j}} e^{(s+\sigma) z+\varphi(z)+\psi(z)} .
\end{aligned}
$$

b) Let $\widetilde{f}_{j \nu}$ be the analogous function to $f_{j \nu}$ which is obtained by replacing $\psi$ by $\widetilde{\psi}$ and $\alpha_{j}$ by $\bar{\alpha}_{j}$. Then we obtain

$$
\begin{aligned}
& \left\langle S \widetilde{T} e_{j \nu}, e_{i \mu}\right\rangle=s_{i \mu}\left(\widetilde{T} e_{j \nu}\right)=\int_{-\infty}^{0} g_{i \mu}(s) \widetilde{f}_{j \nu}(s) d s \\
& \quad=\left.\left.\frac{1}{\left(n_{i}-\mu\right) !} \frac{\partial^{n_{i}-\mu}}{\partial w^{n_{i}-\mu}}\right|_{w=\alpha_{i}} \frac{1}{(\nu-1) !} \frac{\partial^{\nu-1}}{\partial z^{\nu-1}}\right|_{z=\bar{\alpha}_{j}} \frac{e^{\varphi(w)+\widetilde{\psi}(z)}}{w+z} .
\end{aligned}
$$

From the definition of the polynomial $\widetilde{\psi}$, we have $\left.\frac{1}{\kappa !} \frac{\partial^{\kappa}}{\partial z^{\kappa}}\right|_{z=\bar{\alpha}_{j}} e^{\widetilde{\psi}(z)}=\overline{a_{j}^{(\kappa+1)}}$, hence a direct application of the Leibnitz rule yields

$$
\left.\frac{1}{(\nu-1) !} \frac{\partial^{\nu-1}}{\partial z^{\nu-1}}\right|_{z=\bar{\alpha}_{j}} \frac{1}{w+z} e^{\widetilde{\psi}(z)}=\sum_{\nu^{\prime}=0}^{\nu-1}(-1)^{\nu^{\prime}}\left(\frac{1}{w+\bar{\alpha}_{j}}\right)^{\nu^{\prime}+1} \overline{a_{j}^{\left(\nu-\nu^{\prime}\right)}} .
$$

Furthermore, again from the Leibnitz rule,

$$
\begin{aligned}
\left.\frac{1}{\left(n_{i}-\mu\right) !} \frac{\partial^{n_{i}-\mu}}{\partial w^{n_{i}-\mu}}\right|_{w=\alpha_{i}}\left(\frac{1}{w+\bar{\alpha}_{j}}\right)^{\nu^{\prime}+1} e^{\varphi(w)} \\
=\sum_{\mu^{\prime}=0}^{n_{i}-\mu}(-1)^{\mu^{\prime}}\left(\begin{array}{c}
\mu^{\prime}+\nu^{\prime} \\
\mu^{\prime}
\end{array}\right)\left(\frac{1}{\alpha_{i}+\bar{\alpha}_{j}}\right)^{\mu^{\prime}+\nu^{\prime}+1} c_{i}^{\left(\mu+\mu^{\prime}\right)},
\end{aligned}
$$

Inserting C.2 (C.3) into C.1 and substituting summation indices $\mu^{\prime}, \nu^{\prime}$ with $\mu^{\prime}+1, \nu^{\prime}+1$, respectively, we obtain as result

$$
\left\langle S \widetilde{T} e_{j \nu}, e_{i \mu}\right\rangle=\sum_{\mu^{\prime}=1}^{n_{i}-\mu+1} \sum_{\nu^{\prime}=1}^{\nu}(-1)^{\mu^{\prime}+\nu^{\prime}} \overline{a_{j}^{\left(\nu-\left(\nu^{\prime}-1\right)\right)}} c_{i}^{\left(\mu+\left(\mu^{\prime}-1\right)\right)}\left(\begin{array}{c}
\mu^{\prime}+\nu^{\prime}-2 \\
\mu^{\prime}-1
\end{array}\right)\left(\frac{1}{\alpha_{i}+\bar{\alpha}_{j}}\right)^{\mu^{\prime}+\nu^{\prime}-1} .
$$

Finally, comparison with Lemma 7.3 shows that $\left\langle S \widetilde{T} e_{j \nu}, e_{i \mu}\right\rangle$ coincides with the $\mu \nu$ th entry of $C_{i j}$. 
c) As in b) one shows $\bar{C}=\widetilde{S} T$. The factorization $C \bar{C}=(S \widetilde{T} \widetilde{S}) T$ then tells us that $C \bar{C}$ and $H=T(S \widetilde{T} \widetilde{S})$ are related operators. As in a), one verifies that $\widetilde{T} \widetilde{S}$ is the integral operator with kernel

$$
\widetilde{k}(\sigma, s)=\left.\sum_{j=1}^{N} \frac{1}{\left(n_{j}-1\right) !} \frac{\partial^{n_{j}-1}}{\partial z^{n_{j}-1}}\right|_{z=\bar{\alpha}_{j}} e^{(s+\sigma) z+\widetilde{\varphi}(z)+\widetilde{\psi}(z)} .
$$

Moreover,

$$
\widetilde{k}(\sigma, s)=\overline{k(\sigma, s)}
$$

from the definitions of $\widetilde{\varphi}, \widetilde{\psi}$. Now it is straightforward to check that $H=(T S)(\widetilde{T} \widetilde{S})$ is the integral operator with the kernel $h(\sigma, s)$ as claimed.

Observe $k(\sigma, s)=k(s, \sigma)$. Hence $\overline{h(s, \sigma)}=h(\sigma, s)$, implying $(H g, f)=(g, H f)$ for all $f, g \in L_{2}(-\infty, 0]$. Together with the fact that $H$ is bounded, it follows that $H$ is self-adjoint. To show that $H$ is positive, we finally check

$$
\begin{aligned}
(H f, f) & =\int_{-\infty}^{0} \int_{-\infty}^{0} h(\sigma, s) f(s) d s \overline{f(\sigma)} d \sigma \\
& =\int_{-\infty}^{0}\left(\int_{-\infty}^{0} \overline{k(\rho, s)} f(s) d s\right)\left(\int_{-\infty}^{0} k(\sigma, \rho) \overline{f(\sigma)} d \sigma\right) d \rho \\
& =\int_{-\infty}^{0}\left|\int_{-\infty}^{0} \overline{k(\rho, s)} f(s) d s\right|^{2} \geq 0
\end{aligned}
$$

for all $f \in L_{2}(-\infty, 0]$. This completes the proof.

Second part of the proof of Theorem 2.3. According to Section 5 we may assume that Assumptions 3.1 and 3.2 are met. Furthermore, we may assume $\operatorname{Re}\left(\alpha_{j}\right)>0$ for all $j$ according to Appendix B.

Observe that $L=L(x, t)=e^{A x-\mathrm{i} A^{2} t} C$ satisfies the $(x, t)$-dependent Sylvester equation $A L(x, t)+L(x, t) \bar{A}=\widehat{c}(x, t) \bar{a}^{t r}$ for the vector-function $\widehat{c}(x, t)=e^{A x-\mathrm{i} A^{2} t} c$. By Proposition C.1 c), $L \bar{L}$ is related to an integral operator on $L_{2}(-\infty, 0]$ which is positive and therefore has non-negative eigenvalues. From the principle of related operators 16 it follows that also $L \bar{L}$ has only non-negative eigenvalues. Hence

$$
p=\operatorname{det}\left(\begin{array}{cc}
I & -L \\
L & I
\end{array}\right) \stackrel{(2.7)}{=} \operatorname{det}(I+L \bar{L}) \geq 1 .
$$

\section{REFERENCES}

[1] M.J. Ablowitz, B. Prinari, and A.D. Trubatch. 'Discrete and Continuous Nonlinear Schrödinger Systems.' Cambridge University Press, Cambridge, 2003.

[2] M.J. Ablowitz, D.J. Kaup, A.C. Newell, and H. Segur. The inverse scattering transform Fourier analysis for nonlinear problems. Stud. Appl. Math. 53 (1974), 249-315.

[3] M.J. Ablowitz and H. Segur. 'Solitons and the Inverse Scattering Transform.' SIAM, Philadelphia, 1981

[4] H. Aden and B. Carl. On realizations of solutions of the KdV equation by determinants on operator ideals. J. Math. Phys. 37 (1996), 1833-1857.

[5] T. Aktosun, F. Demontis, and C. van der Mee. Exact solutions to the focusing nonlinear Schrödinger equation Inverse Problems 23 (2007), 2171-2195.

[6] H. Blohm. Solution of nonlinear equations by trace methods. Nonlinearity 13 (2000), 19251964.

[7] B. Carl and S.Z. Huang. On realizations of solutions of the KdV equation by the $C_{0}$-semigroup method. Amer. J. Math. 122 (2000), 403-438. 
[8] B. Carl and C. Schiebold. Nonlinear equations in soliton physics and operator ideals. Nonlinearity 12 (1999), 333-364.

[9] B. Carl and C. Schiebold. Ein direkter Ansatz zur Untersuchung von Solitonengleichungen. Jahresber. Deutsch. Math.-Verein. 102 (2000), 102-148.

[10] A. Dimakis and F. Müller-Hoissen Solutions of matrix NLS systems and their discretisations: A unified treatment Inverse Problems 26 (2010), 095007.

[11] W. Eckhaus and A. van Harten. 'The Inverse Scattering Transformation and The Theory of Solitons.' Mathematics Studies 50, North-Holland Publishing Company, Amsterdam 1981.

[12] B. Fuchssteiner and R.N. Aiyer. Multisolitons, or the discrete eigenfunctions of the recursion operator on non-linear evolution equations: II. Background. J. Phys. A 20 (1987), 375-388.

[13] C.S. Gardner, J.M. Greene, M.D. Kruskal, and R.M. Miura. Method for solving the Kortewegde Vries equation. Phys. Rev. Lett. 19 (1967), 1095-1097.

[14] V.A. Marchenko. 'Nonlinear Equations and Operator Algebras.' Reidel, Dordrecht, 1988.

[15] E. Olmedilla. Multiple pole solutions of the non-linear Schrödinger equation. Physica D 25 (1986), 330-346.

[16] A. Pietsch. 'Eigenvalues and s-Numbers.' Cambridge University Press, Cambridge 1987.

[17] C. Pöppe. Construction of solutions of the sine-Gordon equation by means of Fredholm determinants. Physica D 9 (1983), 103-139.

[18] A. L. Sakhnovich Non-self-adjoint Dirac-type systems and related nonlinear equations: wave functions, solutions, and explicit formulas. Integral Equations Operator Theory $\mathbf{5 5}$ (2006), $127-143$.

[19] C. Schiebold. Solitons of the sine-Gordon equation coming in clusters. Revista Matemática Complutense 15 (2002), 265-325.

[20] C. Schiebold. On negatons of the Toda lattice. J. Nonlin. Math. Phys. 10 (2003), 181-193.

[21] C. Schiebold. 'Integrable Systems and Operator Equations.' Habilitation thesis, Jena University, 2004 (http://apachepersonal.miun.se/ corsch/Habilitation.PDF).

[22] C. Schiebold, A non-abelian Nonlinear Schrödinger equation and countable superposition of solitons. J. Gen. Lie Theory Appl. 2 (2008), 245-250.

[23] C. Schiebold. Noncommutative AKNS systems and multisoliton solutions to the matrix sineGordon equation. Discrete and Continuous Dynamical Sytems, Supplement 2009 (2009), 678690.

[24] C. Schiebold. Cauchy-type determinants and integrable systems. Linear Algebra Appl. 433 (2010), 447-475.

[25] C. Schiebold. The noncommutative AKNS system: projection to matrix systems, countable superposition and soliton-like solutions. J. Phys. A 43 (2010), 434030.

[26] J.J. Sylvester. Sur l'équation en matrices $p x=x q$. C. R. Acad. Sci. Paris 99 (1884) 67-71, 115-116.

[27] S. Tanaka. Non-linear Schrödinger equation and modified Korteweg-de Vries equation; Construction of solutions in terms of scattering data. Publ. RIMS Kyoto Univ. 10 (1975), 329357.

[28] H. Tsuru and M. Wadati. The multiple pole solutions of the sine-Gordon equation. J. Phys. Soc. Japan 9 (1984), 2908-2921.

[29] M. Wadati and K. Ohkuma. Multiple pole solutions of the modified Korteweg-de Vries equation. J. Phys. Soc. Japan 51 (1981), 2029-2035.

[30] V.E. Zakharov and A. Shabat. Exact theory of two-dimensional self-focusing and onedimensional self-modulation of waves in nonlinear media Sov. Phys. JETP 34 (1972), 62-69.

E-mail address: Cornelia.Schiebold@miun.se 